\title{
Caring about confusion
}

Citation for published version (APA):

van Velthuijsen, E. L. (2018). Caring about confusion: on the daily practice of diagnosis and management of delirium in older hospitalised patients. [Doctoral Thesis, Maastricht University]. Ridderprint BV. https://doi.org/10.26481/dis.20180704ev

Document status and date:

Published: 01/01/2018

DOI:

$10.26481 /$ dis.20180704ev

Document Version:

Publisher's PDF, also known as Version of record

\section{Please check the document version of this publication:}

- A submitted manuscript is the version of the article upon submission and before peer-review. There can be important differences between the submitted version and the official published version of record.

People interested in the research are advised to contact the author for the final version of the publication, or visit the DOI to the publisher's website.

- The final author version and the galley proof are versions of the publication after peer review.

- The final published version features the final layout of the paper including the volume, issue and page numbers.

Link to publication

\footnotetext{
General rights Owners
rights.

- You may freely distribute the URL identifying the publication in the public portal. please follow below link for the End User Agreement:

www.umlib.nl/taverne-license

Take down policy

If you believe that this document breaches copyright please contact us at:

repository@maastrichtuniversity.nl

providing details and we will investigate your claim.
}

Copyright and moral rights for the publications made accessible in the public portal are retained by the authors and/or other copyright owners and it is a condition of accessing publications that users recognise and abide by the legal requirements associated with these

- Users may download and print one copy of any publication from the public portal for the purpose of private study or research.

- You may not further distribute the material or use it for any profit-making activity or commercial gain

If the publication is distributed under the terms of Article $25 \mathrm{fa}$ of the Dutch Copyright Act, indicated by the "Taverne" license above, 


\section{Caring about confusion}

\section{On the daily practice of diagnosis and management of delirium in older hospitalised patients}

Eveline Louise van Velthuijsen 


\section{Colofon}

The research presented in this dissertation was conducted at the Care and Public Health Research Institute (CAPHRI), department of Health Services Research, Maastricht University. CAPHRI is part of the Netherlands School of Primary Care (CaRe), which has been acknowledged by the Royal Netherlands Academy of Science (KNAW).

This research was funded by the Academic Collaborative Center on Sustainable Care, which is an initiative of Maastricht University Medical Centre+ and Maastricht University.

(C) Eveline van Velthuijsen, Maastricht 2018

Cover design: Mattt Baaij \& Eveline van Velthuijsen

Cover Illustration: Mattt Baaij | www.illumatie.nl

Lay-out: Eveline van Velthuijsen

Printing: Ridderprint BV | www.ridderprint.nl

ISBN 978-94-6375-003-5

All rights reserved. No parts of this thesis may be reproduced or transmitted, in any form or by any means, electronic or mechanical, including photocopying, recording or otherwise, without prior written permission of the holder of the copyright. 


\title{
Caring about confusion
}

\section{On the daily practice of diagnosis and management of delirium in older hospitalised patients}

\author{
Proefschrift \\ ter verkrijging van de graad van doctor aan de Universiteit Maastricht, \\ op gezag van de Rector Magnificus, Prof. dr. Rianne M. Letschert \\ volgens het besluit van het College van Decanen, \\ in het openbaar te verdedigen \\ op woensdag 4 juli 2018 om 16:00 uur \\ door
}

Eveline Louise van Velthuijsen 


\section{Promotores}

Prof. dr. S.M.G. Zwakhalen

Prof. dr. G.I.J.M. Kempen

Prof. dr. F.R.J. Verhey

\section{Beoordelingscommissie}

Prof. dr. R.P. Koopmans (voorzitter)

Prof. dr. B.M. Buurman (Academisch Medisch Centrum, Amsterdam)

Dr. J.F.B.M. Fiolet

Prof. dr. R.C. van der Mast (Leids Universitair Medisch Centrum)

Prof. dr. J.M.G.A. Schols 


\section{Contents}

$\begin{array}{llr}\text { Chapter } 1 & \text { General Introduction } & 7\end{array}$

Chapter 2 Psychometric properties and feasibility of instruments for the 21

detection of delirium in older hospitalised patients: a

systematic review

Chapter 3 Detection and management of hyperactive and hypoactive

delirium in older patients during hospitalisation: a

retrospective cohort study evaluating daily practice

Chapter 4 Effects of a medication review on delirium in older

hospitalised patients: a comparative retrospective cohort study

Chapter 5 Can education improve clinical practice concerning delirium in older hospitalised patients? Results of a pre-test post-test study on an educational intervention for nursing staff

Chapter 6 Managing hypoactive delirium: should or should we not use antipsychotics?

Chapter 7 General discussion

Addenda Summary

Samenvatting

Valorisation

Acknowledgments

Publications and curriculum vitae 
Er verscheen een wezenloze uitdrukking op zijn gelaat terwijl verwarde gedachten zijn brein doorkruisten.

"Wat bedoelt hij met: In orde?"

Prevelde hij tenslotte.

- $\quad$ Marten Toonder (Olivier B. Bommel, 1971)

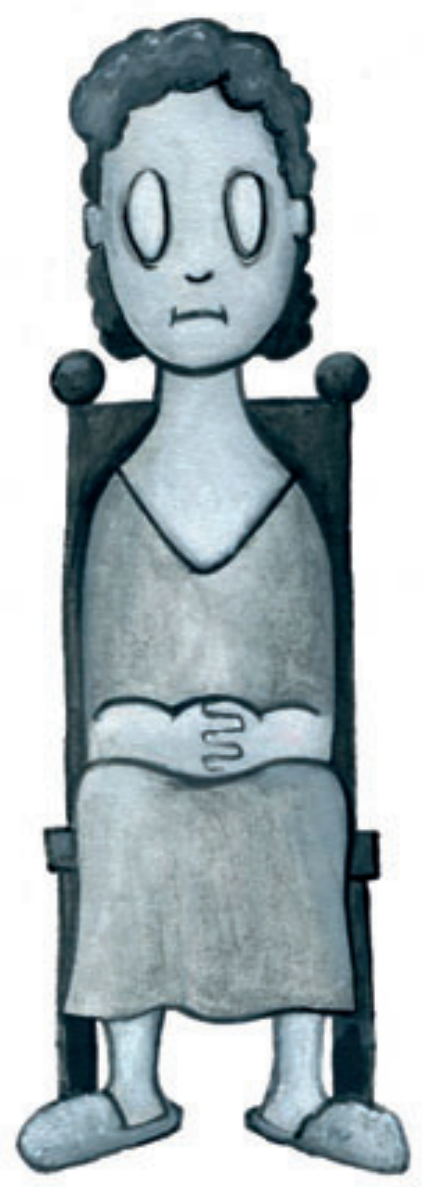




\section{CHAPTER 1 \\ General introduction}


Mr. Smit is an 85-year old widower, who lives alone. He sees his children and grandchildren regularly, plays bridge two times a week with friends and walks his dog three times a day. He has no memory problems and he is in fairly good health, though he has some health issues: congestive heart failure and he suffered from a stroke five years ago. He has medication for both and takes them appropriately. One day, while walking his dog in the park, mr. Smit tripped, fell and broke his hip. He was rushed to the hospital, where he underwent surgery for the broken hip. When mr. Smit woke up from the anaesthesia, he was a bit groggy, received tramadol for the pain and had a urinary catheter, but felt generally well, considering the circumstances. He was confident that he would recover swiftly.

During the first night in the hospital, mr. Smit started to feel disoriented; he was not quite sure where he was. He couldn't seem to remember the nurses' faces, and started to feel insecure. Then, he started to see bugs crawling on the walls and ceilings, coming towards him. Mr. Smit felt scared and wanted to get away from the bugs. He tried to get out of bed, but fell again because of his hip, and screamed. A nurse walks in to see what the noise is all about, and she finds mr. Smit in a state of alarm and confusion on the floor next to his bed. Luckily, no additional damage has emerged from the fall, but mr. Smit refuses to go back to bed and resists the nurses' attempts to help him. With the help of a doctor and a second nurse, mr. Smit is finally helped into bed, where he is sedated for the rest of the night, and bed rails are put up to prevent him from leaving the bed again. It is discovered $\mathrm{mr}$. Smit has a urinary tract infection, probably as a result of the catheter. Mr. Smit is given antibiotics, and the infection goes away. Together with the abating infection, mr. Smit's confusion clears up, and after two days he starts to feel more like himself again.

Mr. Smit suffered a delirium as a result of a urinary tract infection (UTI). His story, though distressing, is a very common one. Older hospitalised patients run a high risk of developing a delirium: it affects between $29 \%$ and $64 \%$ of all hospitalised patients 65 years and older. ${ }^{1}$ Delirium was first described over 2500 years ago by Hippocrates as an acute mental disorder associated with severe illness, fever, and head trauma ${ }^{2}$. He used the words phrenitis (frenzy) and lethargus (lethargy) to describe the agitated and apathic types of delirium. The word delirium originates from the Latin de lira, which literally means "to deviate from the straight track" and was first recorded in the first century A.D. ${ }^{2}$ The disorder has been fairly consistently and accurately described since the time of Hippocrates. However, despite its long track-record, delirium remains poorly understood, and is often missed or misdiagnosed. ${ }^{3}$ Apart from the burden to the patient, delirium can also be a heavy burden for the patient's family and also causes heavy financial burdens on the health care system. ${ }^{1}$ Moreover, delirium is preventable in about $30-40 \%$ of the patients. ${ }^{4,5}$ The preventability in combination with high costs associated with delirium makes this a very relevant disorder to study. 


\section{Delirium symptoms and subtypes}

Delirium is an acute brain failure as a result of physical illness. Delirium is characterised by an acute change in consciousness and a decline in cognitive functions with reduced ability to focus, sustain or shift attention. ${ }^{6}$ These symptoms tend to fluctuate over the course of the day. ${ }^{6}$ The disorder is also often accompanied by perceptual disturbances, such as audio-visual hallucinations and delusions, and motor symptoms, such as agitation or slowing of movement and apathy. ${ }^{6}$

These above mentioned motor symptoms form the basis of the different delirium subtypes. Originally, a distinction was made between two subtypes of delirium: hyperactive and hypoactive. ${ }^{7}$ As the name implies, hyperactive delirium is characterised by motor agitation, hyperactivity and hypervigilance. These patients are restless, fidgety, may try to get out of bed, interfere with medical equipment and may become verbally or physically aggressive, forming a threat to themselves and/or others. ${ }^{8,9}$ Hypoactive delirium, on the other hand, is characterised by a slowing of motor activity and speech, apathy, and somnolence.8,9 Although patients with a hypoactive delirium seem very calm and quiet, they can also suffer from hallucinations and delusions, which can be equally distressing as those experienced by patients with a hyperactive delirium. ${ }^{10}$ Patients can also shift between hyperactive and hypoactive delirium; this is referred to as a mixed delirium. 8,9 Lastly, some patients experience delirium with only the cognitive and perceptual symptoms and do not show signs of motor agitation or apathy. ${ }^{11}$ This is referred to as delirium without motor symptoms. To date, it remains unclear what causes these different motor symptoms in delirium.

Although hyperactive delirium is the most easily recognised and the most "visible" of the subtypes, the hypoactive subtype is actually the most common form of delirium, with prevalence rates between 31\% and 71\%. ${ }^{11-14}$ However, hypoactive delirium often remains undetected or unreported, patients are often perceived as being cooperative and exhibit fewer behavioural problems, making a hypoactive delirium difficult to detect. ${ }^{11,15,16}$ Studies on the prognosis of the different subtypes remain inconclusive. Though most studies seem to find that the hypoactive subtype has the worst prognosis, ${ }^{17-20}$ other studies find this to be true for the hyperactive subtype $^{21,22}$ or don't find any significant differences. ${ }^{23}$ Moreover, it is not clear whether these differences in prognosis are due to the delirium subtype itself, the underlying causes of the delirium, or because of the (often) delayed recognition and treatment of the hypoactive subtype.

\section{Epidemiology and consequences of delirium}

The prevalence rates of delirium among older hospitalised patients vary strongly between different hospital wards. The Intensive Care Unit (ICU) generally shows the highest incidence rates, with up to $82 \%$ of the patients developing a delirium 
during their ICU stay. General medical wards also show high occurrence rates of delirium: between $29 \%$ and $64 \% .^{1}$

The adverse outcomes associated with delirium can be very severe. Mortality has been found to be associated strongly with delirium in hospitalised patients. ${ }^{24,25}$ Older patients suffering a delirium during hospitalisation run a $62 \%$ increased risk of mortality within twelve months after the hospital admission compared to patients who did not develop a delirium. ${ }^{26}$ Other adverse outcomes include increased length of hospital stay, slower functional recovery, cognitive decline, institutionalisation after hospital discharge, greater chance of hospital readmissions, recurring delirium, and onset of dementia or deterioration of preexistent dementia. ${ }^{27-30}$ On top of the adverse physical outcomes, patients also suffer from emotional or psychological distress after a delirious episode. ${ }^{31}$ After recovering from a delirium, patients have been found to suffer from symptoms of depression, anxiety, and fear, 32,33 and delirium may be a risk factor for the development of post-traumatic stress disorder (PTSD). ${ }^{34}$

\section{Economic and social burden}

The adverse outcomes mentioned above do not only have a negative effect on the patient, but also place a heavy financial burden on the health care system and the society. Longer and more hospital admissions, long-term care institutionalisation, dementia; these are all adverse outcomes with high financial consequences. It has been calculated that delirium among older hospitalised patients costs more than US $\$ 164$ billion a year in the USA, which is comparable to the healthcare costs for falls or diabetes mellitus. ${ }^{35}$ A German study team calculated what a patient with a hyperactive delirium actually costs the hospital (i.e. does not include the costs for re-admissions or institutionalisation); ${ }^{36}$ they found that patients with a hyperactive delirium costs on average $€ 1200$,- more than patients with no delirium, based on extra time spent by physicians and nurses, longer hospital stay, and additional medication costs.

In addition to the patient's personal burden and the burden on health care costs, delirium can also cause significant problems for a patient's family and the medical staff. Interview studies have found that family members of delirious patients suffer substantially at seeing their family member in a delirious state; ${ }^{37,38}$ and some say they no longer recognise the patient. ${ }^{39}$ Medical staff also report feelings of stress and anxiety, as well as physical and emotional burden related to caring for delirious patients. ${ }^{37-42}$ For the nursing staff, the main concerns are the physical manifestations of the disorder: patients getting out of bed, yelling, keeping up other patients on the wards, pulling at medical equipment, and in some cases also verbal or physical aggression. ${ }^{43}$ 


\section{Risk factors and causes of delirium}

The risk factors for delirium can be divided into two categories: predisposing and precipitating factors. Predisposing factors are those which are inherent to the patient and cannot be changed or avoided, such as age, dementia, or comorbidities. Precipitating factors are factors which are not inherent to the patient and could potentially be avoided, but which trigger a delirium in a patient already vulnerable to delirium, such as medication use, surgery or an infection. Although delirium can be caused by a single predisposing or precipitating factor, this is rarely the case. Rather, delirium is mostly multifactorial, caused by a combination of predisposing and precipitating factors. This model for delirium risk is called the multifactorial model of delirium in older age, and was developed by Inouye and Charpentier ${ }^{4}$ (see Figure 1). The Figure shows that people with many predisposing factors are highly vulnerable and a small noxious insult could be enough to trigger a delirium, whereas for people with just a few predisposing factors and low vulnerability, a more serious noxious insult is required to trigger a delirium.

If we apply this model to the patient described at the beginning of this chapter, mr. Smit, we notice that he had low or moderate vulnerability: he had no dementia or other cognitive problems, reasonable health, lead an active life, though his age and previous stroke do make him a bit more vulnerable for delirium. A fall followed by surgery, tramadol, a UTI and a sudden change of environment (from home to the hospital), all combine into a strong noxious insult which triggered a delirium in $\mathrm{mr}$. Smit.

\section{Predisposing factors / vulnerability}

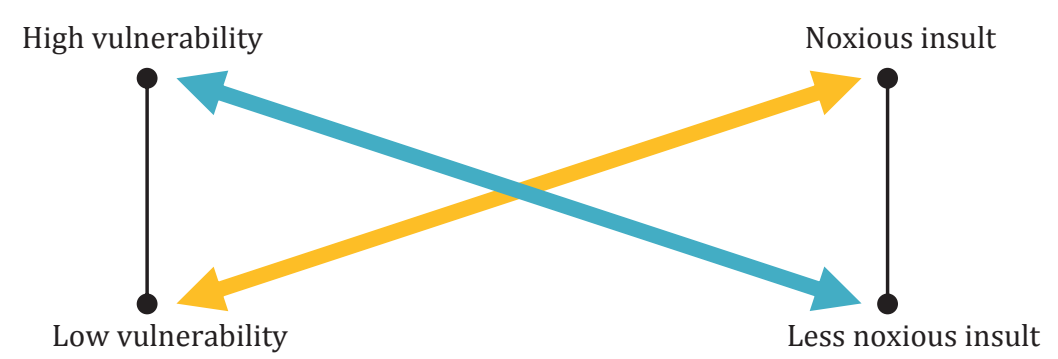

Figure 1. The multifactorial model of delirium from Charpentier and Inouye

There are many different predisposing and precipitating factors for delirium, and they have been extensively studied. Predisposing factors include older age $(\geq 65$ years), dementia, visual and hearing impairments, comorbidity, depression, stroke or transient ischaemic attack, and a previous delirium. ${ }^{45}$ Precipitating factors include polypharmacy, the use of psychoactive, hypnotic or sedative medications, the use of a bladder catheter, infections (e.g. UTI or pneumonia), surgery, 
dehydration, malnutrition, the use of physical restraints, and any other iatrogenic event. ${ }^{1,45,46}$

\section{Detection and differential diagnosis of delirium}

Delirium is a clinical diagnosis, and can best be made through clinical observations and a (brief) cognitive assessment of the patient. The current standard diagnostic criteria for delirium are the fifth edition of the Diagnostic and Statistical Manual of Mental Disorders (DSM-V) of the American Psychiatric Association, ${ }^{6}$ and the tenth version of the International Classification of Diseases (ICD-10) of the World Health Organisation (WHO). ${ }^{47}$ Despite it being so common, delirium is often missed or misdiagnosed: ${ }^{3}$ between $19 \%$ and $64 \%$ of all older hospitalised patients suffer a delirium, yet only between $2,5 \%$ to $5 \%$ of patients are diagnosed or reported in the patient's medical files. ${ }^{48,49}$

This under-recognition of delirium in older patients is problematic because delirium is like the proverbial canary in a coalmine: it is a warning for an often serious underlying physical problem such as an infection or dehydration. Timely recognition of a delirium can therefore speed up the recognition and treatment of the underlying ailment. Moreover, studies have found that a shorter delirious episode can lead to faster and better functional recovery. ${ }^{50}$ Thus, timely and adequate recognition of delirium can have a positive impact on a patients' recovery. Many instruments have been developed to improve the recognition of delirium. The most widely used instrument is the Confusion Assessment Method (CAM), developed by Inouye and colleagues. ${ }^{51}$ In the Netherlands, the most commonly used screening instrument is the Delirium Observation Screening score (DOS), developed by Schuurmans and colleagues. ${ }^{52}$ Electroencephalography (EEG) is also being considered as a more objective way of diagnosing delirium through altered brain waves. ${ }^{53}$

Despite the availability of adequate screening instruments, it can be challenging to differentiate delirium from dementia or depression in older patients, and a patient can have a delirium superimposed on dementia or a depression. ${ }^{54}$ The differentiation between these disorders is vital, however, as the cause, treatment and prognoses of all three are fundamentally different. Delirium is always caused by an underlying physical problem, and the delirium will often clear up once the underlying cause is treated. For depression and dementia, this is not the case. Incorrectly identifying a delirium as a depression or dementia therefore, can lead to failing to treat the underlying cause, creating a potentially harmful situation for the patient and unnecessarily prolonging the delirious episode. Moreover, people with dementia may not be able to return home after their hospitalisation, whereas a patient who suffered a delirium could be able to go home once the patient has recovered physically and the delirious episode has passed. In the case of mr. Smit, the diagnosis was less challenging, as there were no signs of cognitive impairment at the time of hospitalisation, and the delirium resolved quickly once the underlying cause had been treated, ruling out both dementia and depression. 


\section{Prevention and treatment}

\section{Prevention}

Many of the risk factors for developing a delirium in the hospital, such as immobility, UTI due to an (unnecessary) urinary catheter, and polypharmacy are iatrogenic. ${ }^{45}$ Avoiding these risk factors can potentially prevent delirium in roughly $30-40 \%$ of the older patients. 4,5 Multicomponent non-pharmacologic interventions have repeatedly and consistently shown to be the most effective in preventing delirium among hospitalised older adults. 4,55-60 These multicomponent preventive interventions generally include education of the medical and nursing staff, early mobilisation, adequate hydration and nutrition, sleep hygiene protocols, orientation strategies (i.e. providing a clock and calendar), optimisation of hearing and vision by making sure all hearing and visual aids are used properly, and cognitive stimulation activities. Due to the often multifactorial causes of delirium, the non-pharmacologic interventions work best when implemented together by a multidisciplinary team, but show little effect when the components are implemented separately. ${ }^{61}$

Pharmacologic delirium prophylaxis is an often used strategy for preventing delirium in older hospitalised patients. The evidence supporting this practice, however, is weak and circumstantial.58,62-64 Some studies find that prophylaxis using antipsychotic medication can prevent postoperative delirium. ${ }^{58}$ However, methodological limitations such as small sample sizes, lack of randomisation, no blinding, and exclusion of patients with dementia increase potential bias and severely limit the reliability and validity of these findings, $58,61,62,65$ especially considering that dementia is one of the most important precipitating factors for delirium. Moreover, the use of antipsychotics in older hospitalised patients can lead to negative side effects, including increased QT-times and increased risk of extrapyramidal side effects (EPS). ${ }^{65-67}$

\section{Treatment}

As noted above, delirium is a consequence of an underlying physical problem. Thus, the first course of action should always be to find and cure what is causing the delirium.1,65 This would include taking the patient's medical history, neurological and physical examination, and laboratory tests (e.g. electrolyte balance and check for infections). In the case of mr. Smit, the delirium was caused by a UTI, and treating the UTI also resulted in curing the delirium. However, in many cases the delirium is multifactorial (i.e. not caused by a single factor) or the precipitating factor is not something that can be cured, such as dementia. In these cases, all other modifiable factors that could also precipitate a delirium should be checked. Additionally, all delirious patients should receive a medication review to delete any unnecessary or risky medication and avoid negative medication interactions. ${ }^{65}$ 
As delirium is generally a multifactorial disorder, it is best treated with a multicomponent intervention focussing on all possible modifiable factors, both physical and environmental.65 Important factors are maintaining a healthy sleepwake cycle, hydration, nutrition, mobilisation (i.e. taking a patient out of bed), orientation (using a clock and calendar). 1,65 The most commonly used intervention is the Hospital Elder Life Program (HELP), developed by Inouye and colleagues. ${ }^{4}$ This program combines all above mentioned interventions, and also includes education for nursing staff and actively involves family members in caring for the patient, as this can have a calming effect.

Pharmacological interventions mostly include administering antipsychotic medication such as haloperidol. The evidence for use of haloperidol for delirium is debated, as there is little evidence to support this practice and the available evidence is generally weak and circumstantial. ${ }^{58,62,66}$ International guidelines on delirium in hospitalised patients advise to use medication only in case a patient poses a threat for himself or others due to severe agitation or behavioural problems, and only after all the above mentioned non-pharmacological interventions have been tried without the intended effect. ${ }^{68,69}$ The Dutch guidelines are, however, less strict when it comes to using antipsychotic medication for delirium, stating it can also be used to treat the hallucinations or delusions in delirious patients even if there are no signs of the patient posing a threat to himself or others. ${ }^{70,71}$ This lack of consensus on whether and when to use antipsychotic medication for the treatment of delirium is problematic: it shows there is still a dearth of information regarding the treatment of delirium and creates confusion among physicians about the optimal treatment for their patients.

\section{Objectives and outline of the dissertation}

This dissertation focuses on the daily hospital practice regarding the recognition and management of delirium in older hospitalised patients. We evaluate current practices and guidelines, and try to improve specific aspects of delirium care in daily hospital practice. The questions that formed the basis of this work were: What are the current guidelines for the recognition and management of delirium in older hospitalised patients? How and how well are these guidelines being implemented in practice? How can we improve the current practice regarding recognition and management of delirium in older hospitalised patients? What are the current interventions in the hospital for the management of delirium, and are they effective?

The information presented in the previous sections of this chapter provide the status quo in delirium care for older hospitalised patients, but many questions and hiatus in knowledge still remain. Some of these questions play a key role in this dissertation: What is the best instrument for diagnosing delirium in daily hospital practice? How often are the different types of delirium detected in everyday 
hospital practice, and how are they managed? Is a medication revision an effective tool to manage delirium in older hospitalised patients? Can educating the nursing staff increase delirium recognition and improve the management of delirium? And lastly, should patients with a hypoactive delirium receive antipsychotic medication to subdue any hallucinations or delusions that they might have?

The questions formulated above are divided into several chapters in this dissertation. Chapter two provides the results of a systematic literature review on the available instruments for the recognition of delirium in older hospitalised patients, taking into account the psychometric properties but also the feasibility for use in daily clinical of these instruments. Chapter three is a retrospective cohort study which gives insight into how well hyperactive and hypoactive delirium in older hospitalised patients is recognised and managed in an academic hospital setting. Chapter four goes on to explore one specific intervention for delirium management, namely the medication review. By comparing patients who had a delirium before medication reviews were introduced to patients who had a delirium after introduction of a medication review, we could study the effects of this intervention on different aspects of delirium. In chapter five we describe the development and implementation of an educational programme for nurses about delirium in older hospitalised patients, and examined the effects of this programme on clinical practice. In chapter six we contribute to the discussion of whether a hypoactive delirium without behavioural or physical problems should be treated using antipsychotic medication, by making use of the available national and international guidelines and evidence from studies performed in this field. The final chapter discusses the main findings and implications of the studies in this dissertation next to some methodological and theoretical considerations. Lastly, recommendations for clinical practice and future research are presented. 


\section{References}

1. Inouye SK, Westendorp RGJ,Saczynski JS: Delirium in elderly people. Lancet 2014; 383:911-922

2. Adamis D, Treloar A, Martin FC, et al: A brief review of the history of delirium as a mental disorder. Hist Psychiatry 2007; 18:459-469

3. Collins N, Blanchard MR, Tookman A, et al: Detection of delirium in the acute hospital. Age Ageing 2010; 39:131-135

4. Inouye SK, Bogardus ST, Charpentier PA, et al: A multicomponent intervention to prevent delirium in hospitalized older patients. N Engl J Med 1999; 340:669-676

5. Marcantonio ER, Flacker JM, Wright RJ, et al: Reducing delirium after hip fracture: a randomized trial. J Am Geriatr Soc 2001; 49:516-522

6. American Psychiatric Association: Diagnostic and Statistical Manual of Mental Disorders (4th ed.), 4. Washington, DC, American Psychiatric Association, 2000

7. Lipowski ZJ: Transient cognitive disorders (delirium, acute confusional states) in the elderly. Am J Psychiat 1983; 140:1426-1436

8. Meagher DJ: Motor subtypes of delirium: past, present and future. Int Rev Psychiatr 2009; 21:59-73

9. Ross CA, Peyser CE, Shapiro I, et al: Delirium: phenomenologic and etiologic subtypes. Int Psychogeriatr 1991; 3:135-147

10. Breitbart W, Gibson C,Tremblay A: The delirium experience: Delirium recall and delirium-related distress in hospitalized patients with cancer, their spouses/caregivers, and their nurses. Psychosomatics 2002; 43:183-194

11. Albrecht JS, Marcantonio ER, Roffey DM, et al: Stability of Postoperative Delirium Psychomotor Subtypes in Individuals with Hip Fracture. J Am Geriatr Soc 2015; 65:970-976

12. Boettger S,Breitbart W: Phenomenology of the subtypes of delirium: phenomenological differences between hyperactive and hypoactive delirium. Palliat Support Care 2011; 9:129-135

13. Meagher DJ, Leonard M, Donnelly S, et al: A longitudinal study of motor subtypes in delirium: frequency and stability during episodes. J Psychosom Res 2012; 72:236-241

14. Stagno D, Gibson C,Breitbart W: The delirium subtypes: a review of prevalence, phenomenology, pathophysiology, and treatment response. Palliat Support Care 2004; 2:171-179

15. Inouye SK, Foreman MD, Mion LC, et al: Nurses' recognition of delirium and its symptoms: comparison of nurse and researcher ratings. Arch Intern Med 2001; 161:2467-2473

16. Rice KL, Bennett M, Gomez M, et al: Nurses' recognition of delirium in the hospitalized older adult. Clin Nurse Spec 2011; 25:299-311

17. Kiely DK, Jones RN, Bergmann MA, et al: Association between psychomotor activity delirium subtypes and mortality among newly admitted post-acute facility patients. J Gerontol A Biol Sci Med Sci 2007; 62:174-179

18. Robinson TN, Raeburn CD, Tran ZV, et al: Motor subtypes of postoperative delirium in older adults. Arch Surg 2011; 146:295-300 
19. Meagher DJ, Leonard M, Donnelly S, et al: A longitudinal study of motor subtypes in delirium: relationship with other phenomenology, etiology, medication exposure and prognosis. J Psychosom Res 2011; 71:395-403

20. Bellelli G, Speciale S, Barisione E, et al: Delirium subtypes and 1-year mortality among elderly patients discharged from a post-acute rehabilitation facility. J Gerontol A Biol Sci Med Sci 2007; 62:1182-1183

21. Marcantonio E, Ta T, Duthie E, et al: Delirium severity and psychomotor types: their relationship with outcomes after hip fracture repair. J Am Geriatr Soc 2002; 50:850-857

22. Kobayashi K, Takeuchi 0 , Suzuki M, et al: A retrospective study on delirium type. Jpn J Psychiatry Neurol 1992; 46:911-917

23. Slor CJ, Adamis D, Jansen RW, et al: Delirium motor subtypes in elderly hip fracture patients: risk factors, outcomes and longitudinal stability. J Psychosom Res 2013; 74:444-449

24. Buurman BM, Hoogerduijn JG, de Haan RJ, et al: Geriatric conditions in acutely hospitalized older patients: prevalence and one-year survival and functional decline. PLOS ONE 2011; 6:e26951

25. Pitkala KH, Laurila JV, Strandberg TE, et al: Prognostic significance of delirium in frail older people. Dement Geriatr Cogn Disord 2005; 19:158-163

26. Leslie DL, Zhang Y, Holford TR, et al: Premature death associated with delirium at 1-year follow-up. Arch Intern Med 2005; 165:1657-1662

27. Witlox J, Slor CJ, Jansen RW, et al: The neuropsychological sequelae of delirium in elderly patients with hip fracture three months after hospital discharge. Int Psychogeriatr 2013; 25:1521-1531

28. Witlox J, Eurelings LS, de Jonghe JF, et al: Delirium in elderly patients and the risk of postdischarge mortality, institutionalization, and dementia: a metaanalysis. JAMA 2010; 304:443-451

29. Krogseth M, Watne LO, Juliebo V, et al: Delirium is a risk factor for further cognitive decline in cognitively impaired hip fracture patients. Arch Gerontol Geriatr 2016; 64:38-44

30. Zenilman ME: Delirium: An Important Postoperative Complication. JAMA 2017; 317:77-78

31. O'Malley G, Leonard M, Meagher D, et al: The delirium experience: A review. J Psychosom Res 2008; 65:223-228

32. Davydow DS: Symptoms of depression and anxiety after delirium. Psychosomatics 2009; 50:309-316

33. Langan C, Sarode DP, Russ TC, et al: Psychiatric symptomatology after delirium: a systematic review. Psychogeriatrics 2017;

34. Drews T, Franck M, Radtke FM, et al: Postoperative delirium is an independent risk factor for posttraumatic stress disorder in the elderly patient: a prospective observational study. Eur J Anaesthesiol 2015; 32:147-151

35. Leslie DL, Marcantonio ER, Zhang Y, et al: One-year health care costs associated with delirium in the elderly population. Arch Intern Med 2008; 168:27-32

36. Weinrebe W, Johannsdottir E, Karaman M, et al: What does delirium cost? : An economic evaluation of hyperactive delirium. Z Gerontol Geriatr 2015; 
37. Toye C, Matthews A, Hill A, et al: Experiences, understandings and support needs of family carers of older patients with delirium: a descriptive mixed methods study in a hospital delirium unit. Int J Older People Nurs 2013;

38. Partridge JSL, Martin FC, Harari D, et al: The delirium experience: What is the effect on patients, relatives and staff and what can be done to modify this? Int J Geriatr Psychiatry 2013; 28:804-812

39. Stenwall E, Sandberg J, Eriksdotter Jönhagen M, et al: Relatives' experiences of encountering the older person with acute confusional state: Experiencing unfamiliarity in a familiar person. Int J Older People Nurs 2008; 3:243-251

40. Bélanger L,Ducharme F: Patients' and nurses' experiences of delirium: A review of qualitative studies. Nurs Crit Care 2011; 16:303-315

41. Clissett P, Porock D, Harwood RH, et al: Experiences of family carers of older people with mental health problems in the acute general hospital: A qualitative study. J Adv Nurs 2013; 69:2707-2716

42. Pollard C, Fitzgerald M,Ford K: Delirium: the lived experience of older people who are delirious post-orthopaedic surgery. Int J Ment Health Nurs 2015; 24:213-221

43. Mc Donnell S,Timmins F: A quantitative exploration of the subjective burden experienced by nurses when caring for patients with delirium. J Clin Nurs 2012; 21:2488-2498

44. Inouye SK,Charpentier PA: Precipitating factors for delirium in hospitalized elderly persons. Predictive model and interrelationship with baseline vulnerability. JAMA Psychiatry 1996; 275:852-857

45. Ahmed S, Leurent B,Sampson EL: Risk factors for incident delirium among older people in acute hospital medical units: a systematic review and metaanalysis. Age Ageing 2014; 0:1-8

46. Oh ES, Li M, Fafowora TM, et al: Preoperative risk factors for postoperative delirium following hip fracture repair: a systematic review. Int J Geriatr Psychiatry 2015; 30:900-910

47. World Health Organisation: International Statistical Classification of Diseases and Related Health Problems (ICD-10), Geneva 2010

48. Rooney S, Qadir M, Adamis D, et al: Diagnostic and treatment practices of delirium in a general hospital. Aging Clin Exp Res 2014; 26:625-633

49. McCoy TH, Jr., Snapper L, Stern TA, et al: Underreporting of Delirium in Statewide Claims Data: Implications for Clinical Care and Predictive Modeling. Psychosomatics 2016;

50. Kiely DK, Jones RN, Bergmann MA, et al: Association between delirium resolution and functional recovery among newly admitted postacute facility patients. J Gerontol A Biol Sci Med Sci 2006; 61:204-208

51. Inouye SK, van Dyck $\mathrm{CH}$, Alessi CA, et al: Clarifying confusion: the confusion assessment method. A new method for detection of delirium. Ann Int Med 1990; 113:941-948

52. Schuurmans MJ, Shortridge-Baggett LM,Duursma SA: The Delirium Observation Screening Scale: a screening instrument for delirium. Res Theory Nurs Pract 2003; 17:31-50

53. van der Kooi AW, Zaal IJ, Klijn FA, et al: Delirium detection using EEG: what and how to measure. Chest 2015; 147:94-101 
54. Downing LJ, Caprio TV,Lyness JM: Geriatric psychiatry review: differential diagnosis and treatment of the 3 D's - delirium, dementia, and depression. Curr Psychiatry Rep 2013; 15:365

55. Inouye SK: Prevention of delirium in hospitalized older patients: Risk factors and targeted intervention strategies. Ann Med 2000; 32:257-263

56. Milisen K, Lemiengre J, Braes T, et al: Multicomponent intervention strategies for managing delirium in hospitalized older people: systematic review. J Adv Nurs 2005; 52:79-90

57. Martinez F, Tobar C,Hill N: Preventing delirium: should non-pharmacological, multicomponent interventions be used? A systematic review and metaanalysis of the literature. Age Ageing 2015; 44:196-204

58. Siddiqi N, Harrison JK, Clegg A, et al: Interventions for preventing delirium in hospitalised non-ICU patients. Cochrane Db Syst Rev 2016; 3:CD005563

59. Vidán MT, Sánchez E, Alonso M, et al: An intervention integrated into daily clinical practice reduces the incidence of delirium during hospitalization in elderly patients. J Am Geriatr Soc 2009; 57:2029-2036

60. Zaubler TS, Murphy K, Rizzuto L, et al: Quality improvement and cost savings with multicomponent delirium interventions: replication of the Hospital Elder Life Program in a community hospital. Psychosomatics 2013; 54:219-226

61. Oh ES, Fong TG, Hshieh TT, et al: Delirium in Older Persons: Advances in Diagnosis and Treatment. JAMA 2017; 318:1161-1174

62. Neufeld KJ, Yue J, Robinson TN, et al: Antipsychotic Medication for Prevention and Treatment of Delirium in Hospitalized Adults: A Systematic Review and Meta-Analysis. J Am Geriatr Soc 2016;

63. Lonergan E, Britton AM, Luxenberg J, et al: Antipsychotics for delirium. Cochrane Db Syst Rev 2007; CD005594

64. Oldham MA, Flanagan NM, Khan A, et al: Responding to Ten Common Delirium Misconceptions With Best Evidence: An Educational Review for Clinicians. J Neuropsychiatry Clin Neurosci 2017;

65. Marcantonio ER: Delirium in Hospitalized Older Adults. N Engl J Med 2017; 377:1456-1466

66. Schrijver EJ, de Graaf K, de Vries OJ, et al: Efficacy and safety of haloperidol for in-hospital delirium prevention and treatment: A systematic review of current evidence. Eur J Intern Med 2016; 27:14-23

67. Boettger S, Jenewein J,Breitbart W: Haloperidol, risperidone, olanzapine and aripiprazole in the management of delirium: A comparison of efficacy, safety, and side effects. Palliat Support Care 2015; 13:1079-1085

68. National Institute for Care and Health Excellence: Delirium: prevention, diagnosis and management (NICE guideline CG103), 2010

69. American Psychiatric Association: Practice guidelines for the treatment of patients with delirium, 2010

70. Nederlandse Vereniging voor Klinische Geriatrie: Richtlijn Delier Volwassenen. 2013;

71. Leentjens AF, Molag ML, Van Munster BC, et al: Changing perspectives on delirium care: the new Dutch guideline on delirium. J Psychosom Res 2014; $77: 240-241$ 
If it looks like a duck, swims like a duck,

and quacks like a duck, then it probably is a duck.

- The duck test for abductive reasoning (author unknown)

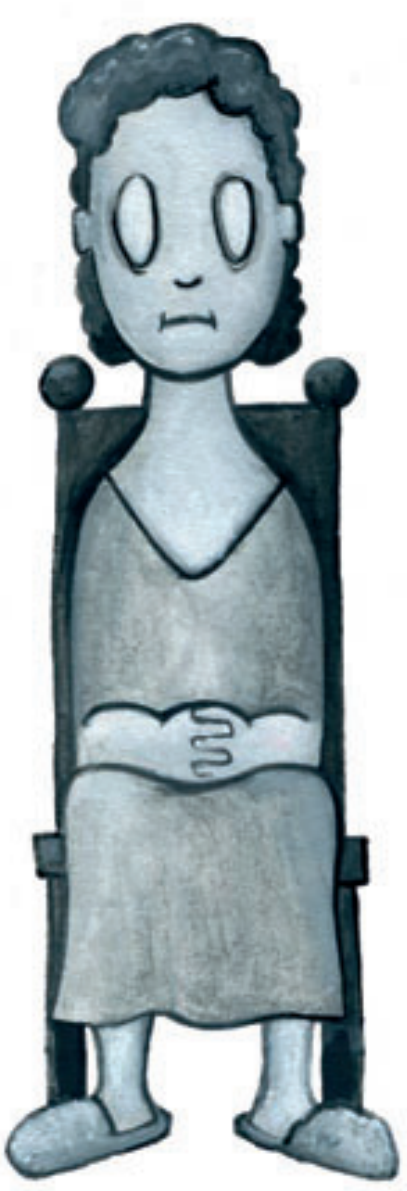




\section{CHAPTER 2 Psychometric properties and feasibility of instruments for the detection of delirium in older hospitalised patients: a systematic review}

This chapter was published as:

Eveline L van Velthuijsen, Sandra MG Zwakhalen, Ron MJ Warnier, Wubbo J Mulder, Frans RJ Verhey and Gertrudis IJM Kempen. Psychometric properties and feasibility of instruments for the detection of delirium in older hospitalized patients: a systematic review. International journal of geriatric psychiatry. 2016; 31(9) 


\begin{abstract}
Objective: Delirium is a serious and common complication among older hospitalised patients and is a predictor of many adverse outcomes. However, up to $72 \%$ of delirium incidents are unrecognised or misdiagnosed. The aim of this systematic review is to determine the validity, reliability and feasibility of instruments for the detection of delirium in older hospitalised patients.

Methods: A systematic literature search was conducted. The inclusion criteria were a mean or median age of 65+ years, the use of the DSM or ICD-10 as a reference standard, and publication in English. All included studies underwent a quality assessment (QUADAS-2). Results: Forty-three of the 3.790 identified studies were relevant to the review, describing 28 instruments. Quality assessment resulted in 37 studies with a positive quality assessment, describing 23 instruments. Five instruments (DOS, NuDESC, CAM, CAM-ICU and DRS-R-98) were described in three or more methodologically sound studies. The DOS and NuDESC are observational instruments with good psychometric properties, but the NuDESC is shorter and has been validated in more languages. The CAM, CAM-ICU, and DRS-R-98 are instruments with both observational and interactive components. The CAM is the most widely studied and demonstrates the best psychometric properties.

Conclusion: Timely detection of delirium might reduce the negative outcomes of delirium in the long term. The NuDESC and CAM appear to be the most adequate instruments for detecting delirium.
\end{abstract}




\section{Introduction}

Delirium is a reversible impairment of the metabolism in the brain with substantial neuropsychiatric symptoms, such as alterations in levels of consciousness, acute problems with memory and attention, orientation and language problems, and hallucinations. ${ }^{1}$ It is a serious complication and is common among older (i.e., those aged 65 years and over) hospitalised patients, with incidence rates of up to $50 \%{ }^{2}$ In addition, delirium is associated with several adverse outcomes including prolonged hospital stay and increased healthcare costs, increased risk of dementia and long-term institutionalisation, long-term cognitive and functional decline, and higher mortality rates within 12 months after hospital discharge. ${ }^{3}$ Early detection facilitates treatment and is therefore important for minimising the duration and adverse effects of the disorder. ${ }^{4}$ Although screening for delirium is a standard procedure in many hospitals, up to $72 \%$ of all delirium incidents are undetected or misdiagnosed. ${ }^{5}$ Possible reasons for this failure include a lack of time, a lack of knowledge of this condition, and a lack of feasible and reliable instruments for the detection of the disorder. The need for an effective detection system that utilises good and feasible instruments is high

In recent years, many instruments have been developed for detecting delirium, especially in older hospitalised patients, but the instruments are not equally valid or reliable, and some require an excessive amount of time to administer. ${ }^{6}$ Although previous reviews have been published, ${ }^{7-9}$ new instruments for the detection of delirium have been developed since, and new validation studies of existing instruments have been published. In addition, the current review takes into account the methodological quality of the validation studies, something that has not been done in previous reviews on this subject. Also, none have focused on detecting delirium specifically in older hospitalised patients, even though this group runs a very high risk of developing delirium and suffering from severe negative effects in the long term. The aim of the present review, therefore, is to provide an overview of the available instruments for the detection of delirium specifically in older hospitalised patients, including the instruments' psychometric properties and feasibility (is the instrument purely observational or does it require interaction with the patient, duration of the assessment, potential raters (i.e., nurses, psychiatrists, geriatricians or other trained hospital staff), simplicity of scoring, ability to determine severity or type of delirium, and costs of the instrument). In contrast to previous reviews, the current review assessed the methodological quality of all included studies and conducted a comprehensive search in four different databases. 


\section{Methods}

\section{Search strategy}

A systematic literature search was conducted on September $14^{\text {th }} 2015$ in the electronic databases PubMed, MEDLINE, PsycINFO and CINAHL for articles concerning instruments for the detection of delirium in older adults in a hospital setting. The search ranged from conception of the database till September $14^{\text {th }}$ 2015. The search combined MeSH terms in PubMed and MEDLINE and thesaurus terms in PsycINFO and CINAHL. A manual search of the reference lists was also performed. The full strategy is shown in Appendix 1.

\section{Inclusion criteria}

The inclusion criteria were the following: 1. reported the psychometric qualities of delirium detection instruments; 2 . aimed at the detection of delirium in older hospitalised patients (mean or median age 65+); and 3. the reference standard was a diagnosis made by an MD based on the criteria of the Diagnostic and Statistical Manual of Mental disorders (DSM, editions III, IV or V) or the International Classification of Diseases (ICD). Apart from age, no restrictions were set for study population or hospital wards. Only articles written in English were considered for inclusion.

\section{Article selection}

First, $10 \%$ of the titles were reviewed independently by authors ELvV and SMGZ. Prior to the review of the articles, it was decided that an agreement of $>95 \%$ between ELvV and SMGZ was acceptable for ELvV to individually complete the screening of the titles. If the disagreement between the two authors was $>5 \%$, the two authors would further specify the inclusion criteria until good agreement on the inclusion criteria was reached. Next, the screening of the abstracts and full-text articles was conducted in the same fashion. In all stages of the article selection, the agreement was $>95 \%$.

\section{Quality assessment}

The methodological quality of the included studies was assessed using the QUADAS-2, a tool that was developed by the Cochrane Review Group ${ }^{10}$ for the quality assessment of diagnostic test accuracy studies (DTAS). Studies are rated as having low, unclear or high risk of bias or concerns regarding applicability for the following four domains: 1. patient selection; 2. index test(s); 3. reference standard; and 4. flow and timing ("high" ratings indicate a high risk of bias and thus lower methodological quality). Authors ELvV and RMJW independently assessed the methodological quality of $50 \%$ of the included studies. As both raters agreed in $>95 \%$ of the evaluations, ELvV assessed the remaining $50 \%$ of the articles. Any 
disagreements between the reviewers were solved by comparing and discussing the findings. Studies were assessed as being of acceptable methodological quality if they scored unclear risk of bias or concern regarding applicability for only one or two of the four domains. Studies scoring a high risk of bias or concern regarding applicability for any domain were not found to be of satisfactory methodological quality.

\section{Classification of instruments}

All instruments were classified as "observational", "interactive", or "mixed", depending on how the instrument is administered. Observational instruments rely on observational data to determine the presence or absence of delirium, and do not require the rater to actively interact with the patient. Interactive instruments rely on information acquired though interaction with the patient, such as an interview or a cognitive test. Mixed instruments rely on both observational and interactive data.

\section{Data extraction}

Data (reference standard, study sample, location (i.e., specific hospital wards), psychometric properties, type (i.e. observational, interactive or mixed), and feasibility of the evaluated instrument) were extracted from the included articles by ELvV. The psychometric properties that were assessed in this review include validity (sensitivity, specificity) and reliability (inter-rater reliability, internal consistency). The feasibility (type of instrument, possible raters, time needed, ability to determine severity or type of delirium, costs involved, necessary training) with respect to daily use in a hospital practice was additionally assessed for each instrument. Data on feasibility is based only on data found in the studies included in the current review, as these are validated specifically for older hospitalised patients.

\section{Results}

The systematic search identified 3.790 unique studies. After careful screening and revision of the titles, abstracts and full text, 43 studies, describing 28 different instruments, met the inclusion criteria for this review (see Figure 1 for reasons of exclusion).

A total of 28 instruments were identified in the included studies. The mean or median age in all studies was $>65$, although 12 studies included participants below 65 years of age (range $17^{11}$ to $100^{12}$ ). The prevalence of delirium ranged from $9 \%{ }^{13,14}$ to $61 \% .^{15}$ Study information such as validated instruments, study population and reference standards can be found in Table 1 . 


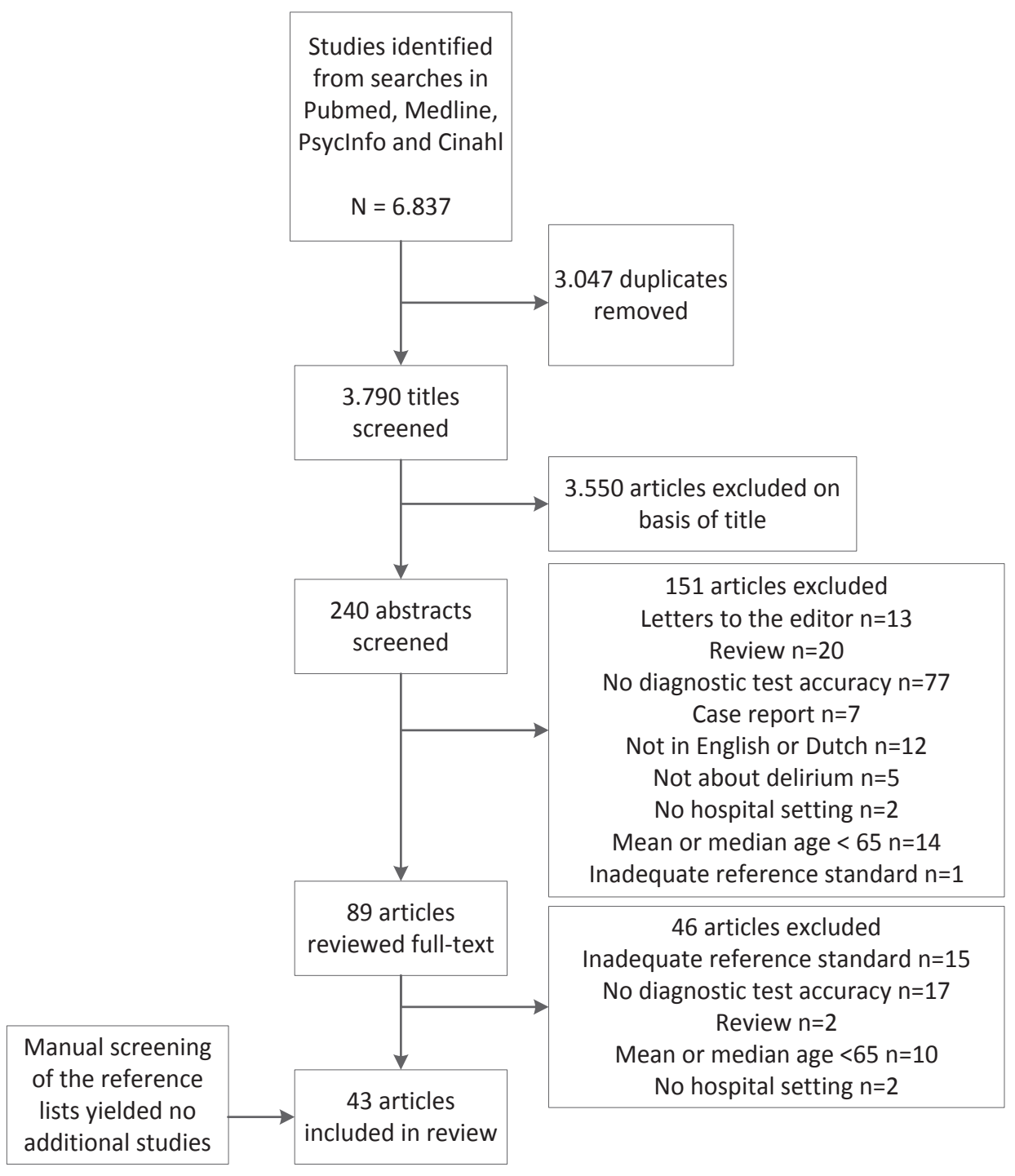

Figure 1. Flowchart of the inclusion process. 
DETECTING DELIRIUM: A SYSTEMATIC REVIEW

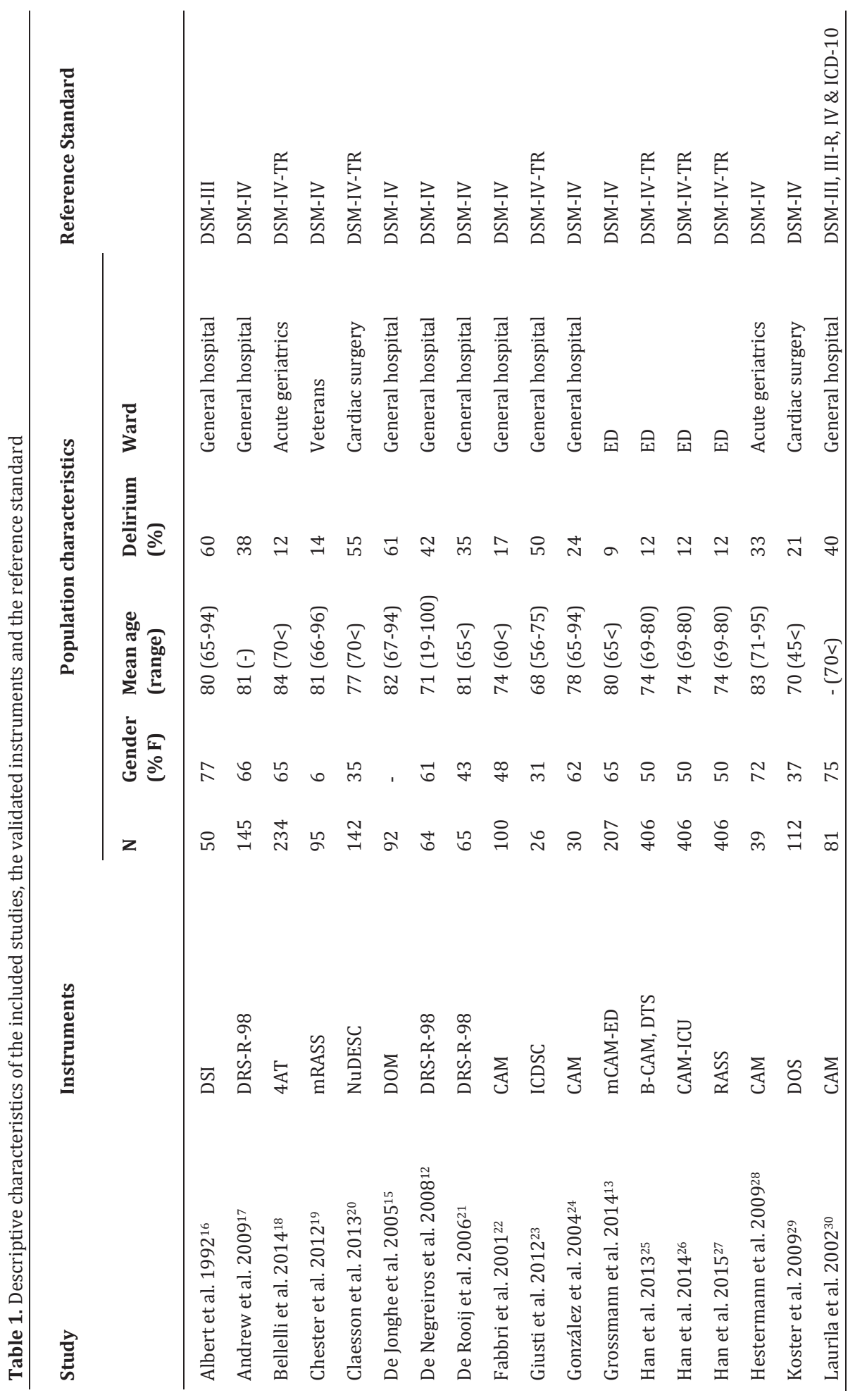




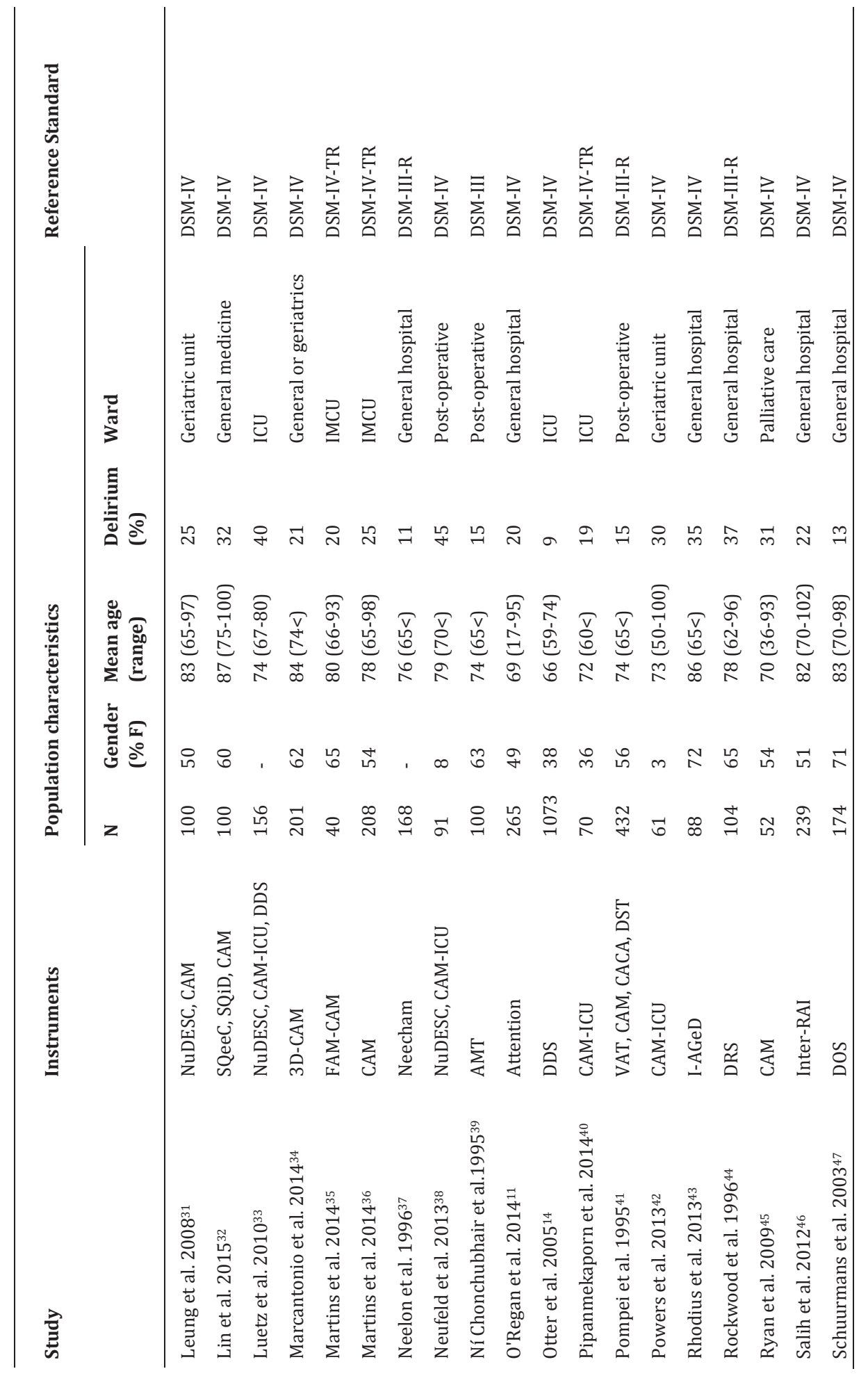




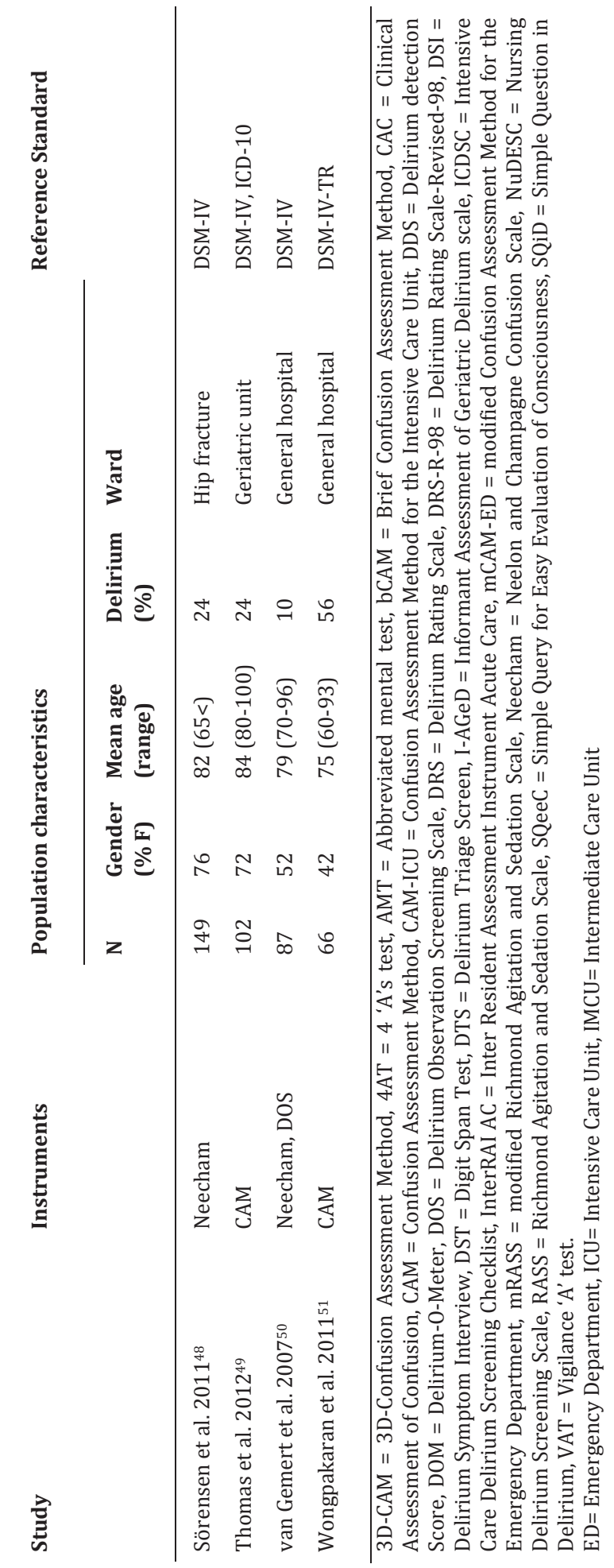




\section{Methodological quality}

The quality assessment assessed 37 out of the 43 studies as having an overall low risk of bias or concerns regarding applicability. The remaining six studies $^{11,13,14,37,41,51}$ were rated as having an overall unclear or high risk of bias or concerns regarding applicability. Table 2 provides an overview of the QUADAS-2 scores per study.

\section{Delirium detection instruments}

From the 43 identified studies, 28 different instruments were identified; 10 observational, 6 interactive and 12 mixed. In addition to the psychometric properties, some instruments can also be used for measuring symptom severity (e.g., DRS-R-98) or type of delirium, (e.g. the DOM). Two instruments were designed for use in the ICU (the CAM-ICU and the ICDSC), and one for use in the emergency department (ED) (e.g. mCAM-ED). Some studies included patients from specific hospital wards, such as the ICU, ${ }^{14,33}$ ED, ${ }^{13,25,52}$ or geriatric wards. ${ }^{18,28,31,42,49}$ Most instruments were designed for use by a trained professional, except the I-AGeD, FAM-CAM and the SQiD, which were designed for use by a family member or an informal carer. The section below summarises the five instruments that were evaluated in three or more studies that were rated as being of acceptable methodological quality. For the psychometric properties and feasibility of all identified instruments, including those not described in the section below, please refer to Tables 3 (psychometric properties) and 4 (feasibility).

Three studies were identified that validated the Delirium Observation Screening scale (DOS). This was originally a 25 -item observational scale, but it was later modified to a 13-item scale. It was developed for use by nurses. Two of the studies included in the current review assessed the original 25-item scale. A score of $>3$ indicates delirium. Sensitivity (89\% and 100\%) and specificity (87\% and 97\%) were calculated in two studies. ${ }^{29,50}$ The third study reported inter-rater reliability (Spearman $r=0.54$ ), and internal consistency (Cronbach's $\alpha=0.93-0.96)$. The DOS has only been validated in Dutch. The 13-item scale is observational, requires approximately five minutes to administer, ${ }^{50}$ and should ideally be administered during three shifts: morning, day and evening. The DOS is available for free, and no specific training for nurses is needed. 


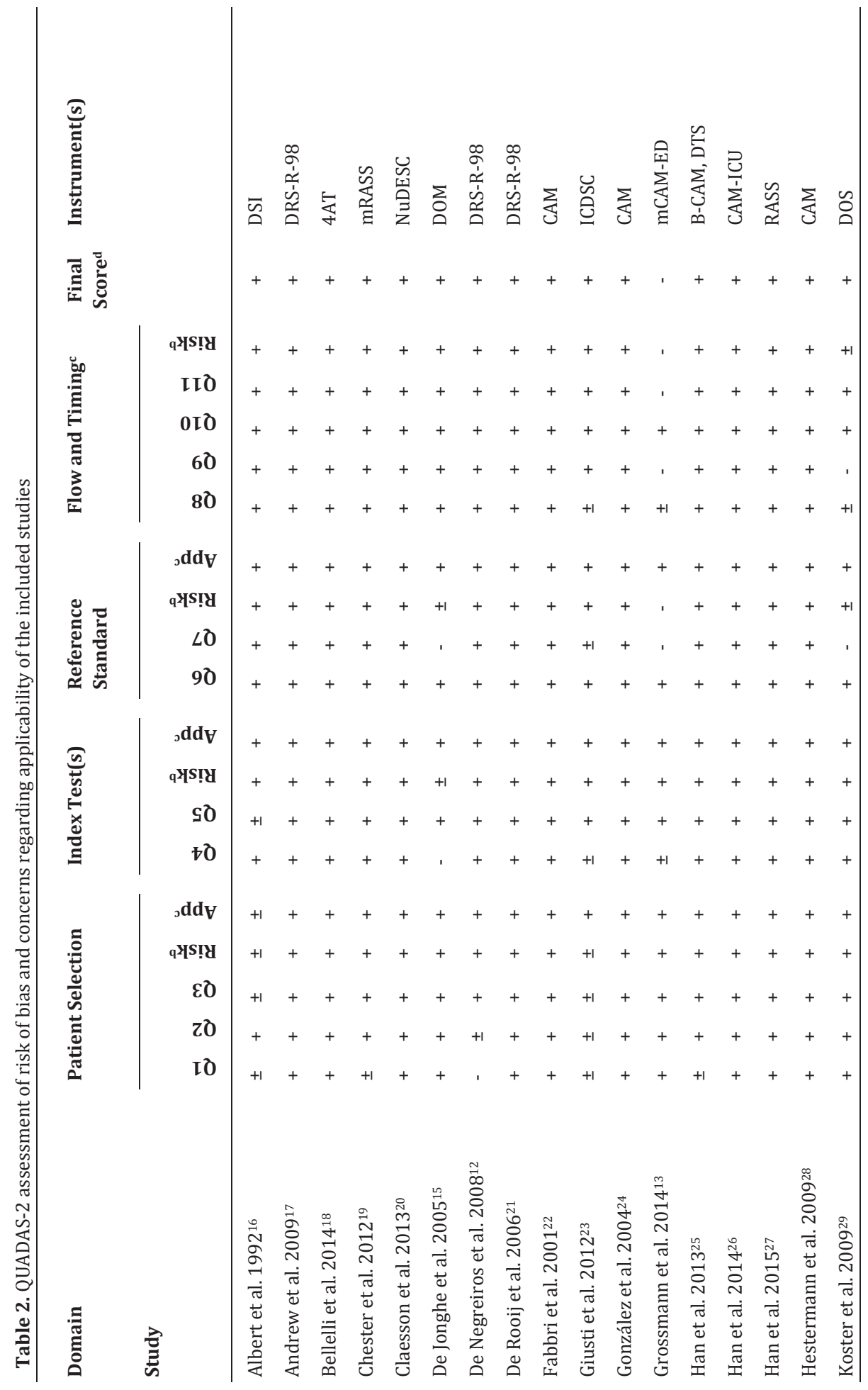




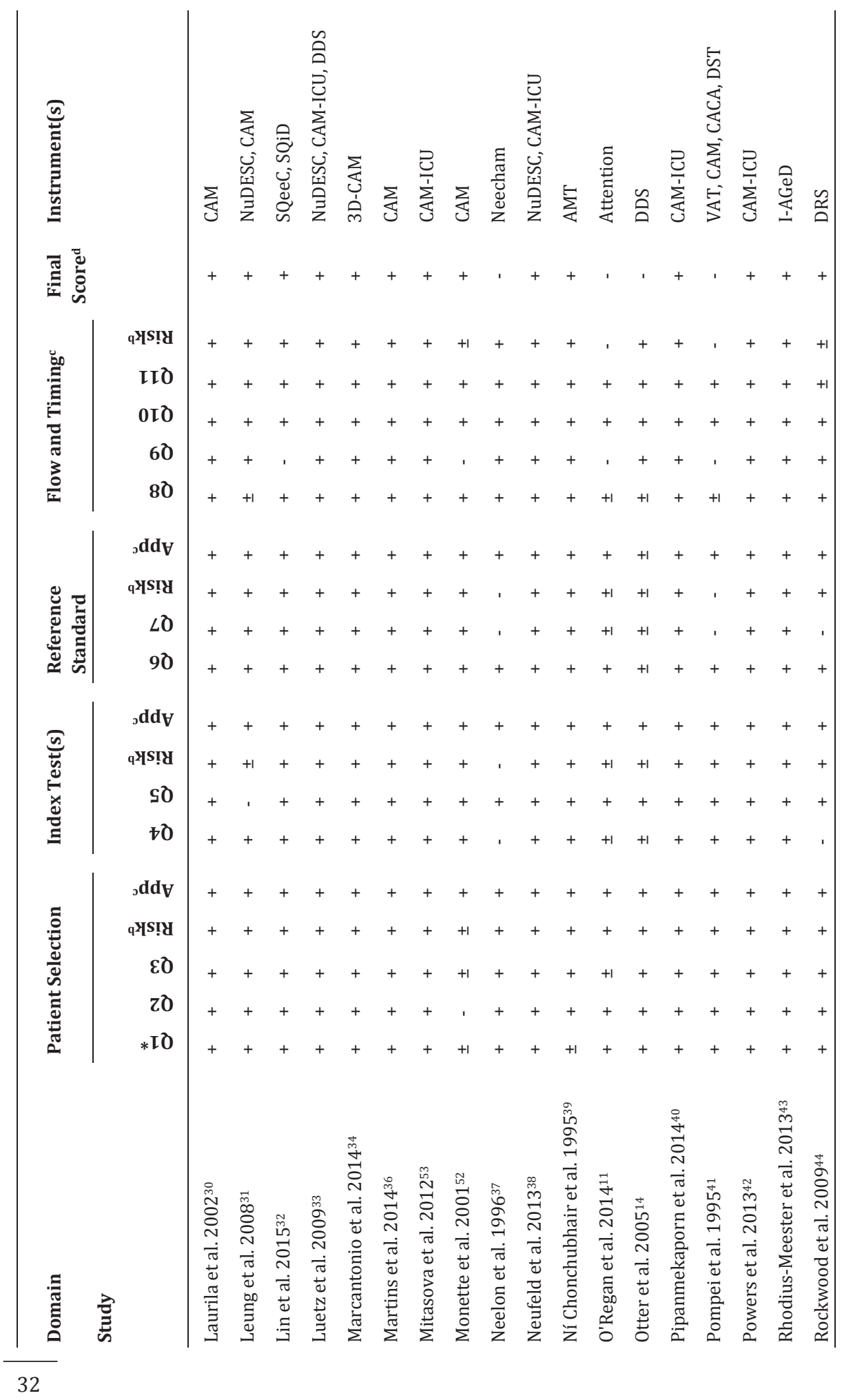




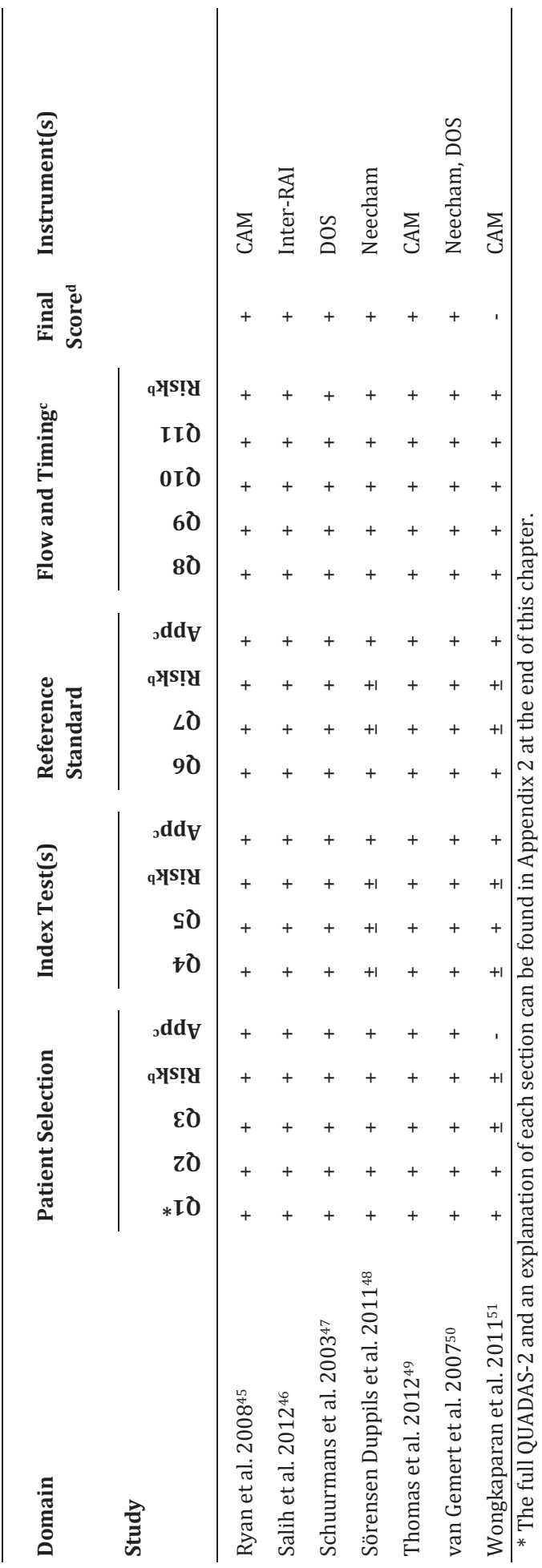




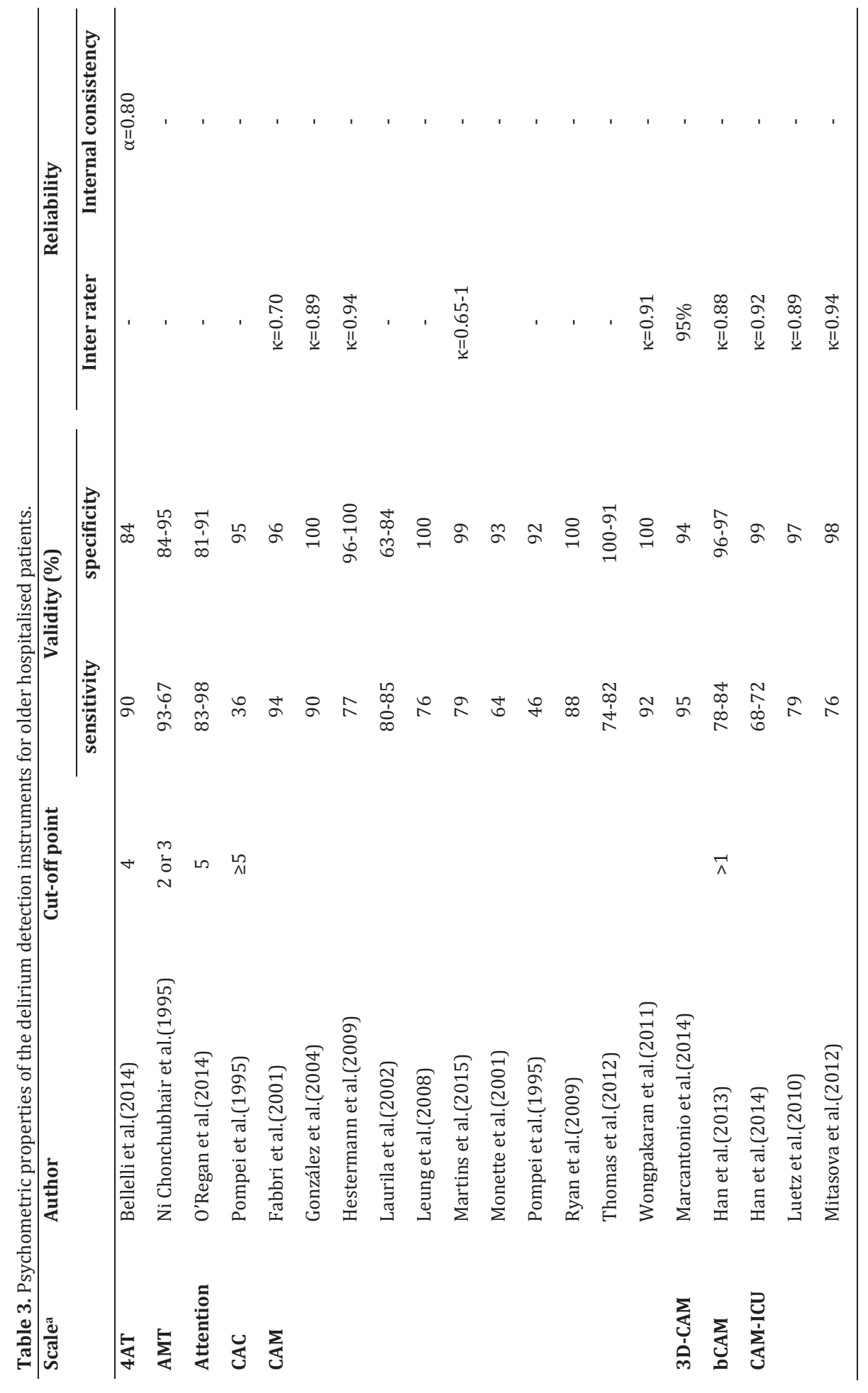




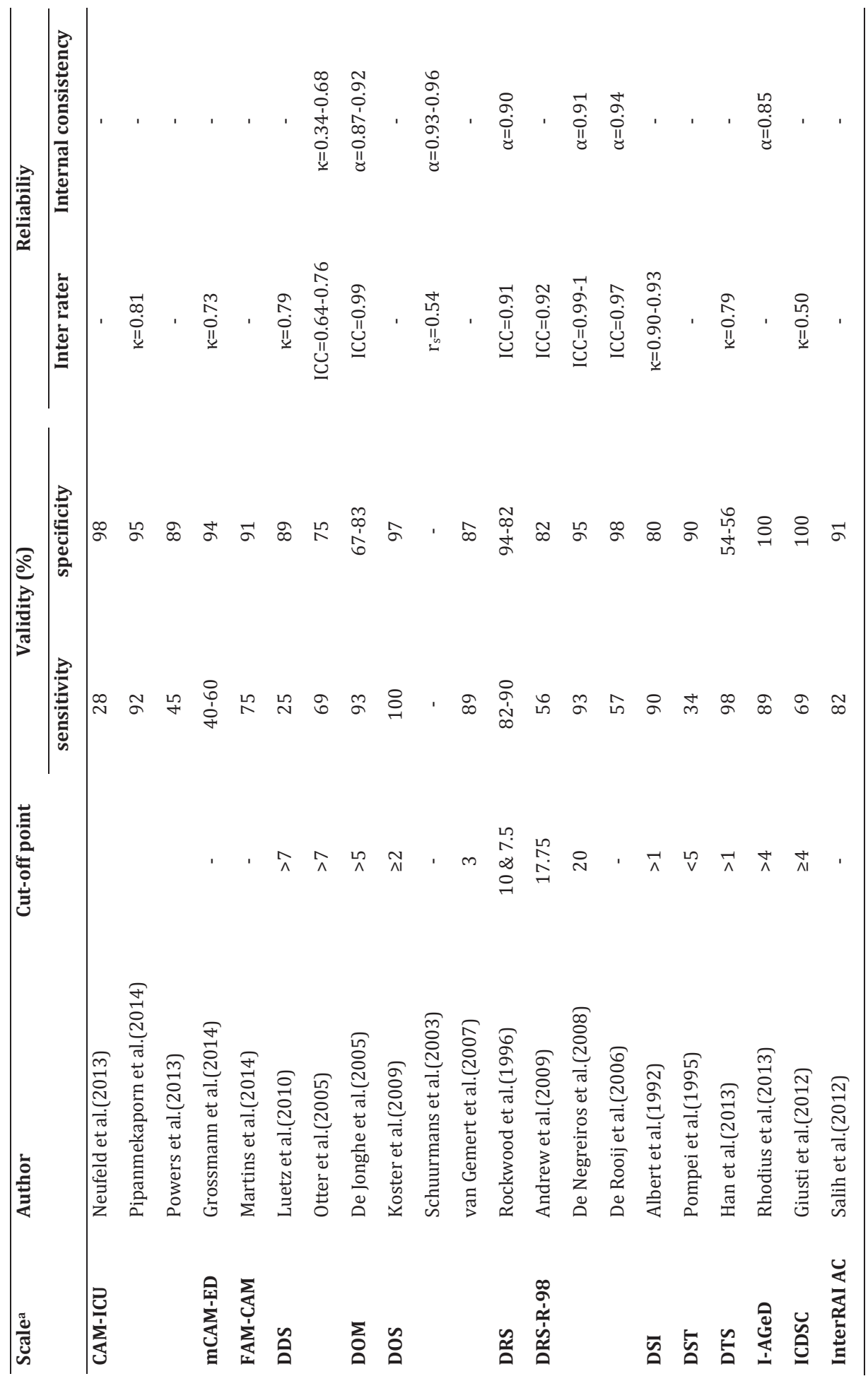




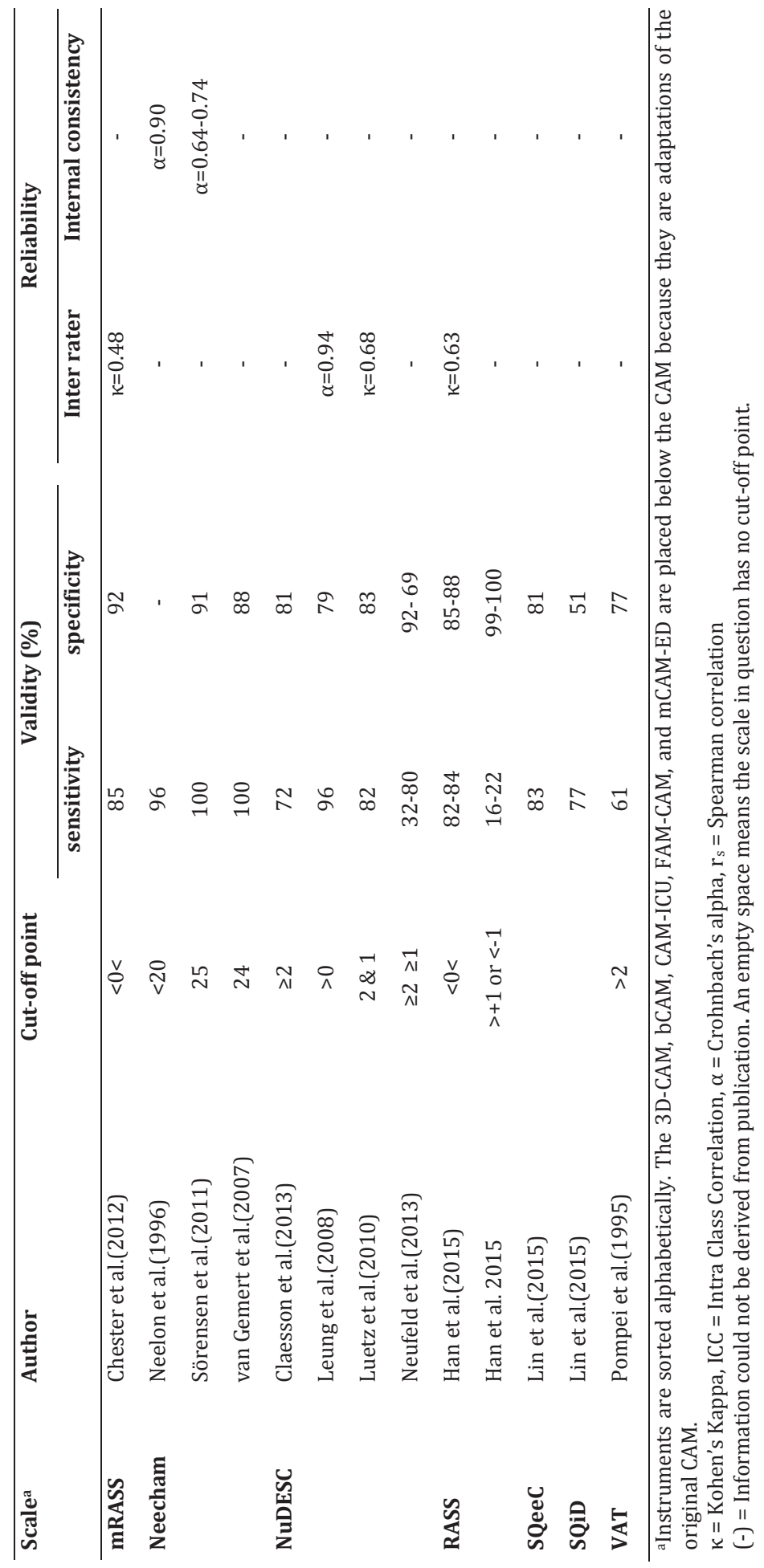




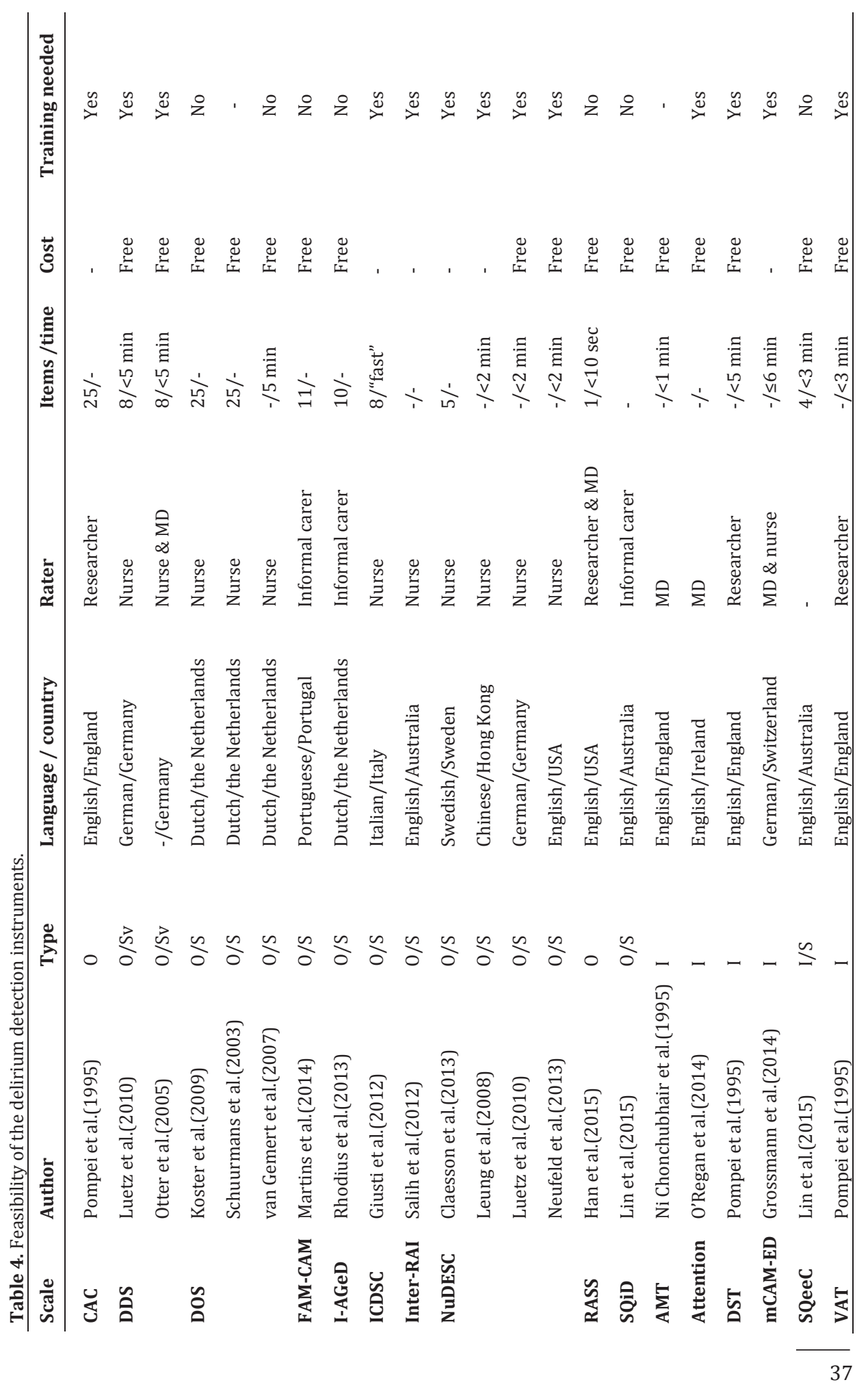




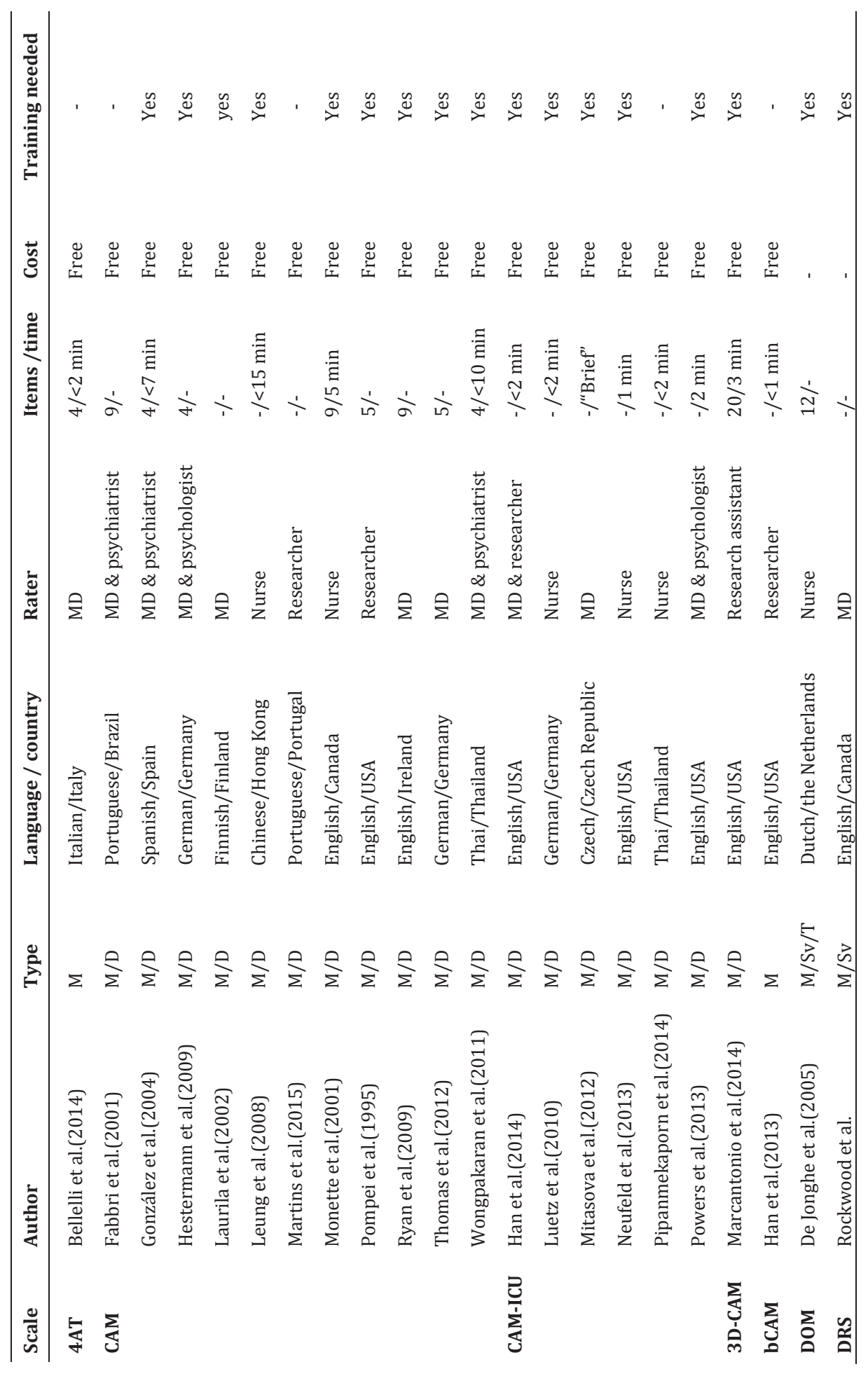




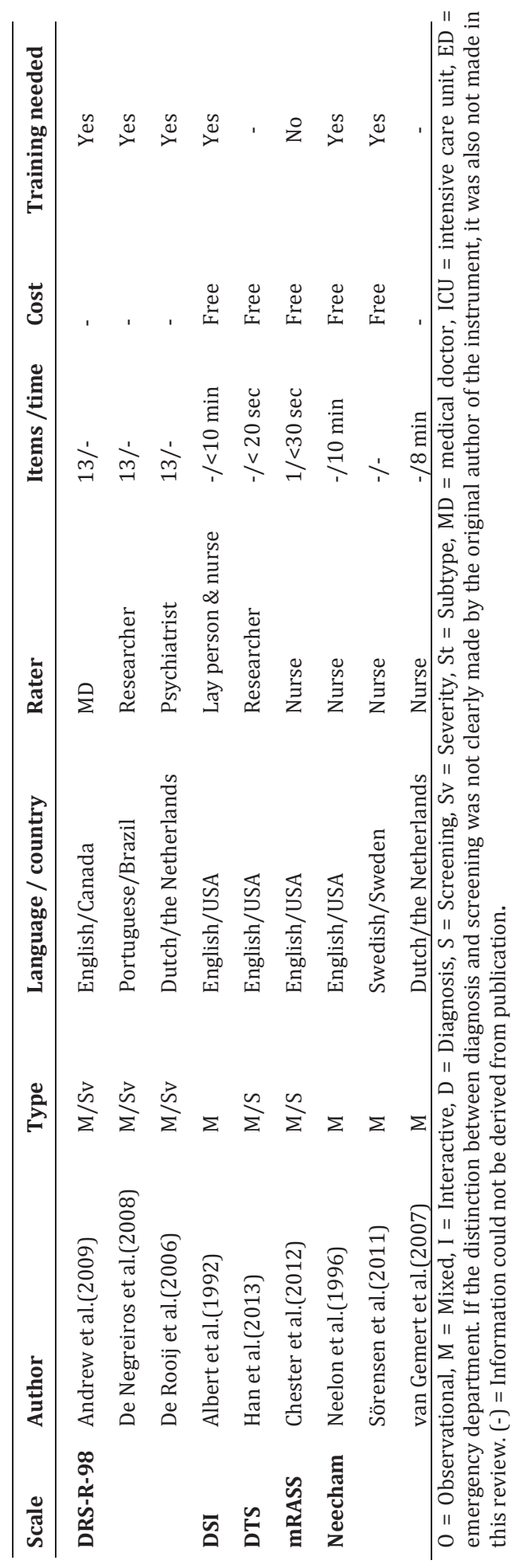


The Nursing Delirium Screening Scale (NuDESC) is an observational instrument that was designed especially for nurses. It has been validated in four studies. The scale has five items: orientation, behaviour, communication, hallucinations, and psychomotor retardation. These items are all rated on a two-point scale, the total score ranges between 0 and 10, where zero indicates no symptoms, and a score of $>2$ indicates delirium. Sensitivity (range 32\% to $96 \%$, with one score below 72\%) and specificity (range $69 \%$ to $92 \%$, with one score below $79 \%$ ) were given in all four studies concerning the NuDESC. However, it must be noted that the highest sensitivity (96\%), was obtained by Leung and colleagues ${ }^{31}$ using a cut-off score of $>0$, where the other studies all used a cut-off score of $>1$ or $>2$. An inter-rater reliability score was calculated in one study $31(\alpha=0.94)$. None of the studies reported scores for internal consistency. The NuDESC has been translated into and validated in four different languages. The scale is observational, and the items are ideally assessed three times per day during each shift (morning, day, and evening) and can be completed in less than two minutes. The scale is available for free, although training is necessary. Apart from uncertainty regarding the cut-off score in one study, ${ }^{31}$ no risks of bias were found.

The Confusion Assessment Method (CAM) was validated in 11 studies. It is a diagnostic tool that is based on the DSM criteria for delirium. It assesses nine different criteria, of which the following four are considered "cardinal": acute onset and fluctuating course; inattention; disorganised thinking; and an altered level of consciousness. Sensitivity (provided for all eleven studies) ranged from $46 \%$ to $94 \%$, although just two studies ${ }^{41,52}$ reported a sensitivity below $74 \%$ (46\% and $64 \%$ respectively). Specificity ranged from $63 \%$ to $100 \%$, though all studies except the one performed by Laurila and colleagues (2002) reported a specificity of $90 \%$ or higher. Inter-rater reliability was provided in four studies (kappa 0.65 to 1.00). Values for internal consistency were not reported. The CAM uses both observational and interactive methods to assess delirium, such as an interview and some cognitive testing. The time necessary to complete the CAM varies from $<7$ to $<15$ minutes. Although the instrument was originally developed for use by nonpsychiatrist clinicians, in two studies the CAM was administered by nurses. ${ }^{31,52}$ It is freely available in seven different languages.

The Confusion Assessment Method-Intensive Care Unit (CAM-ICU) was validated in six studies included in the current review. It is a diagnostic tool that is based on the same four cardinal criteria as the CAM. It was designed specifically for the ICU and can be used with intubated/non-verbal patients. All six studies reported sensitivity (range 28\% to 92\%) and specificity (range 89\% to 99\%). Four studies reported inter-rater reliability values (kappa range 0.81 to 0.94). ${ }^{26,38,40,53}$ Internal consistency was not reported. The CAM-ICU, like the CAM, is scored using both observations and data acquired from cognitive testing. The CAM-ICU has been validated in four different languages, is available for free, and requires two minutes or less to complete. 
The Delirium Rating Scale-Revised-98 (DRS-R-98) was validated in three different studies. It is an adaptation of the original Delirium Rating Scale (DRS), ${ }^{54}$ which was designed for detecting delirium and measuring symptom severity. The scale consists of 16 items, of which three items are intended for detecting delirium, and 13 items are designed for measuring severity. A score of $>18$ indicates delirium. Sensitivity was $56 \%, 57 \%$ and $93 \%$, and specificity was $82 \%, 95 \%$ and $98 \%$. Interrater reliability ranged from 0.92 to 1.00 . Internal consistency was measured in two studies $(\alpha=0.91$ and $\alpha=0.94)$. The DRS-R-98 uses information from both observations and patient interaction. It has been translated into and validated in three different languages. It is designed for use by clinicians (not nurses) and a short training for non-psychiatrists is recommended. No information on the time required for administration, or for costs involved in obtaining the DRS-R-98 was provided in any of the studies.

\section{Instruments for informal caregivers}

In addition to instruments for formal caregivers, three instruments for use by family or informal caregivers were identified: the I-AGeD, FAM-CAM and the SQiD. The I-AGeD is a ten-item yes/no questionnaire based on the DSM-IV criteria for delirium, with a sensitivity of $85 \%$ and a specificity of $100 \%$, though these values are lower when the patient has comorbid dementia. ${ }^{43}$ The FAM-CAM is based on the CAM, and consists of seven yes/no questions, space to provide details about the observed change in the patient, possible fluctuations of the symptoms, and a time frame in which the symptoms have been observed. It has a sensitivity of $75 \%$ and specificity 91\%. ${ }^{35}$ The SQiD ${ }^{32}$ consists of one yes/no question: "Has (name of patient) been more confused lately?" Its sensitivity is $77 \%$ and specificity is $51 \%$.

\section{Other instruments}

The 3D-CAM, 4AT, AMT, bCAM, mRASS, RASS and SQeeC all report a sensitivity and specificity of $80 \%$ or higher (Table 1 ), can be completed within four minutes, and are available for free (Table 2). Furthermore, the studies that included these instruments had a positive assessment on all domains of the QUADAS-2 (Table 3). However, the current review identified just one validation study per instrument. Therefore, these instruments were not described in detail.

\section{Discussion}

A careful review of the literature yielded 43 studies, describing a total of 28 instruments. Thirty-seven studies were of good methodological quality, describing 23 instruments in total. Five instruments (DOS, NuDESC, CAM, CAM-ICU, and DRSR-98) were described in three or more methodologically sound studies. All five instruments appear to be suitable for use in practice, although evidence suggests that the NUDESC seems to be the most appropriate instrument for daily use by 
nurses due to its quick and observation-based nature. The CAM seems to be the best instrument to diagnose and confirm possible cases of delirium due to its generally high sensitivity and excellent specificity. The combination of observations-based and interactive items makes it less feasible for use by nurses in daily practice, but makes it a good diagnostic tool for use by physicians.

The DOS and the NuDESC are both screening instruments based on observations. The DOS has both very high sensitivity and specificity. It was designed specifically for administration by nurses, and requires no extra training before use. It takes about five minutes to administer. However, the DOS has only been validated in Dutch, limiting its use. The NuDESC has equally good psychometric properties, has been more extensively validated, and was also specifically designed for use by nurses. Additionally, the administration of the NuDESC is very quick ( $<2$ minutes), and the instrument has been translated and validated into several different languages, making it more usable than the DOS. Thus, the findings of this review support the use of the NuDESC as a daily observations-based scale for use by nurses to detect delirium in older hospitalised patients.

Previous reviews focusing on delirium detection, 7,8 named the Neecham as the preferred delirium screening instrument. The current review did not find evidence to support that finding. This difference in findings can be explained as follows: 1 . the present review took into account the methodological quality of the included studies, and two out of the three studies validating the Neecham were not found to be of acceptable methodological quality. Thus the authors believe that insufficient evidence is present to support the use of the Neecham; 2. new studies regarding the NuDESC and DOS were published since publication of the previous reviews; and 3. the target population of the present review differs in comparison to previous studies.

The most widely known and validated instrument, the CAM, reports overall high sensitivity and specificity but moderate feasibility because of the amount of time that is required to complete it and because it is preferably administered by a physician. The CAM-ICU was designed specifically for ICU patients. It is also highly specific but has low sensitivity, which could be attributed to its use in ventilated patients. The instrument is based on both observations and patient-interactions, can be administered quickly ( $<2$ minutes) and by a nurse. However, as it was designed specifically for use in the ICU, it may not be generalisable for use in other hospital wards. Due to its high specificity, a positive score for delirium on the CAMICU is nearly diagnostic for delirium, and can be regarded as a good instrument for this specific hospital environment. Further studies would need to be done in nonICU settings to determine its psychometric properties and feasibility outside of the ICU. The DRS-R-98 has been less extensively studied, but studies have also reported good psychometric properties. However, the DRS-R-98 is also partly based on patient-interactions, and preferably administered by a physician. Additionally, no information is available about the time needed to complete the DRS-R-98. Overall, the CAM seems to be the best instrument for diagnosing delirium. The many 
adaptations for use in different settings, such as the CAM-ICU, mCAMed, and the 3DCAM, and possibilities to be used as a screening instrument demonstrate the versatility and robustness of the CAM. These results seem to be in line with previous reviews ${ }^{7-9}$, which all state the CAM as the most appropriate instrument for this purpose.

Several instruments (3D-CAM, 4AT, AMT, bCAM, mRASS, RASS and SQeeC) have not been further validated for the use of detecting delirium in older hospitalised patients following their original validation study, irrespective of their good psychometric properties. These instruments are brief and easy to use, making them more appropriate for integration into clinical care than some other, more complicated or lengthy assessments. However, the scarce amount of evidence currently available on the psychometric properties of these instruments makes them less likely to be used in clinical care. More validation studies assessing the sensitivity, specificity, inter-rater reliability and feasibility of these instruments should be performed to determine their usefulness in clinical practice.

In addition to regular detection instruments for health care professionals, several instruments were identified for use by informal carers: the I-AGeD, FAM-CAM, and SQiD. These instruments can be used alongside a nurse's or physician's assessment and can be used to obtain a clearer picture of the patient's cognitive performance prior to the hospitalisation, aiding the clinician's or nurse's assessment of delirium. Many instruments are designed and used specifically for either screening or diagnosing delirium, but this distinction is not always clear cut. Screening instruments should be highly sensitive, minimising the chance of false negative outcomes. Diagnostic instruments should be highly sensitive, minimise the chance of making a false positive diagnosis. However, the results show that some instruments, such as the DOS, are designed to be screening instruments but have the psychometric properties of a diagnostic instrument (i.e. higher specificity than sensitivity). Therefore, when choosing an instrument for use in a hospital environment, it may not be advisable to choose an instrument based on its intended use as a screening or diagnostic tool, but to look at the specific psychometric properties and feasibility of the individual instruments.

\section{Strengths and limitations}

Some aspects with regard to the methodology of the present review should be addressed. First, this review included only articles that were published in English thus possibly excluding relevant studies in other languages. Second, the concept of delirium underwent alterations since the publication of the DSM-III in 1980 and slightly differs across the diagnostic manuals (DSM-III, IIIR, IV, IV-TR, and the ICD10). Therefore, the operationalisation of the reference standard as specified in this review may differ depending on the reference standard that was used in the studies. This is illustrated by Thomas and colleagues, who found that the sensitivity and specificity of the CAM algorithm varied depending on the reference standard 
that was being used (DSM-IV-TR or the ICD-10). ${ }^{49}$ Third, no distinctions were made between studies including critically ill patients or patients with dementia. As the aim of the current review is to provide an overview of instruments for the screening and diagnosing of older patients in a hospital setting, instruments validated on patients with dementia, and those in the ED or ICU were also included, despite the fact that these patients are notably different from non- demented or non-critically ill patients. Finally, the inclusion criterion for age was a mean or median age of at least 65 years. This led to the inclusion of 12 studies that also studied younger adults. A mean or median age of 65 was chosen to avoid excluding studies that included some "younger" adults but still had a predominantly older patient population. It should also be noted that the administration times reported in the literature may deviate from those in clinical practice. Experienced clinicians and nurses may be quicker and more efficient in completing the instruments in clinical practice than researchers or less experienced clinical personnel in research settings. Therefore the reported time may be overestimated.

The present study has several strengths as well. First, this study provides an up to date overview of the available instruments for detection of delirium, as several new instruments, such as the 3D-CAM, SQiD, and SQeeC have been published recently. Second, an extensive quality check of the methodology of all the included studies was carried out. This quality check allowed the authors to put the results of the validations into perspective and take into account any possible bias that could have affected the psychometric properties of the instruments. Third, through combining psychometric properties, feasibility and methodological quality, a comprehensive assessment of the different instruments was provided.

\section{Conclusion}

Detecting delirium in older hospitalised patients can be challenging but is essential for further treatment and recovery. A sound detection strategy using valid, reliable and feasible instruments such as the CAM or NuDESC might greatly improve recognition rates and, thus, improve delirium care. Several promising new delirium detection instruments have been identified, such as the 3D-CAM, SQiD, and SQeeC, though they still need additional validation. Instruments for use by proxies were also identified, of which the I-AGeD seems the most promising, but also needs further validation to establish their use in clinical practice. 


\section{References}

1. American Psychiatric Association: Diagnostic and Statistical Manual of Mental Disorders (4th ed.), 4. Washington, DC, American Psychiatric Association, 2000

2. Inouye SK, Westendorp RG,Saczynski JS: Delirium in elderly people. Lancet 2014; 383:911-922

3. Eeles EM, Hubbard RE, White SV, et al: Hospital use, institutionalisation and mortality associated with delirium. Age Ageing 2010; 39:470-475

4. Milisen K, Lemiengre J, Braes T, et al: Multicomponent intervention strategies for managing delirium in hospitalized older people: Systematic review. J Adv Nurs 2005; 52:79-90

5. Collins N, Blanchard MR, Tookman A, et al: Detection of delirium in the acute hospital. Age Ageing 2010; 39:131-135

6. Robertsson B, Karlsson I, Styrud E, et al: Confusional State Evaluation (CSE): an instrument for measuring severity of delirium in the elderly. Br J Psychiatry 1997; 170:565-570

7. Adamis D, Sharma N, Whelan PJP, et al: Delirium scales: A review of current evidence. Aging Ment Health 2010; 14:543-555

8. Grover S,Kate N: Assessment scales for delirium: A review. World J Psychiatry 2012; 2:58-70

9. Wong CL, Holroyd-Leduc J, Simel DL, et al: Does this patient have delirium? Value of bedside instruments. JAMA 2010; 304:779-786

10. Whiting PF, Rutjes AW, Westwood ME, et al: QUADAS-2: a revised tool for the quality assessment of diagnostic accuracy studies. Ann Intern Med 2011; 155:529-536

11. O'Regan NA, Ryan DJ, Boland E, et al: Attention! A good bedside test for delirium? J Neurol Neurosurg Psychiatry 2014; 0:1-10

12. de Negreiros DP, Meleiro AMA, Furlanetto LM, et al: Portuguese version of the Delirium Rating Scale-Revised-98: reliability and validity. Int J Geriatr Psychiatry 2008; 23:472-477

13. Grossmann FF, Hasemann W, Graber A, et al: Screening, detection and management of delirium in the emergency department - a pilot study on the feasibility of a new algorithm for use in older emergency department patients: the modified Confusion Assessment Method for the Emergency Department (mCAM-ED). Scand J Trauma Resusc Emerg Med 2014; 22:1-9

14. Otter $\mathrm{H}$, Martin J, Basell K, et al: Validity and reliability of the DDS for severity of delirium in the ICU. Neurocrit Care 2005; 2:150-158

15. de Jonghe JF, Kalisvaart KJ, Timmers JF, et al: Delirium-O-Meter: a nurses' rating scale for monitoring delirium severity in geriatric patients. Int J Geriatr Psychiatry 2005; 20:1158-1166

16. Albert MS, Levkoff SE, Reilly $C$, et al: The delirium symptom interview: an interview for the detection of delirium symptoms in hospitalized patients. J Geriatr Psychiatry Neurol 1992; 5:14-21

17. Andrew MK, Bhat $\mathrm{R}$, Clarke $\mathrm{B}$, et al: Inter-rater reliability of the DRS-R-98 in detecting delirium in frail elderly patients. Age Ageing 2009; 38:241-244 
18. Bellelli G, Morandi A, Davis DH, et al: Validation of the 4AT, a new instrument for rapid delirium screening: a study in 234 hospitalised older people. Age Ageing 2014; 43:496-502

19. Chester JG, Harrington MB,Rudolph JL: Serial administration of a modified Richmond Agitation and Sedation Scale for delirium screening. J Hosp Med 2012; 7:450-453

20. Claesson Lingehall H, Smulter N, Engström KG, et al: Validation of the Swedish version of the Nursing Delirium Screening Scale used in patients 70 years and older undergoing cardiac surgery. J Clin Nurs 2013; 22:2858-2866

21. de Rooij SE, van Munster BC, Korevaar JC, et al: Delirium subtype identification and the validation of the Delirium Rating Scale-Revised-98 (Dutch version) in hospitalized elderly patients. Int J Geriatr Psychiatry 2006; 21:876-882

22. Fabbri RMA, Moreira MA, Garrido R, et al: Validity and reliability of the Portuguese version of the Confusion Assessment Method (CAM) for the detection of delirium in the elderly. Arq Neuropsiquiatr 2001; 59:175-179

23. Giusti GD,Piergentili F: Cultural and Linguistic Validation of the Italian Version of the Intensive Care Delirium Screening Checklist. Dimens Crit Care Nurs 2012; 31:246-251

24. González M, de Pablo J, Fuente E, et al: Instrument for detection of delirium in general hospitals: adaptation of the confusion assessment method. Psychosomatics 2004; 45:426-431

25. Han JH, Wilson A, Vasilevskis EE, et al: Diagnosing delirium in older emergency department patients: validity and reliability of the delirium triage screen and the brief confusion assessment method. Ann Emerg Med 2013; 62:457-465

26. Han JH, Wilson A, Graves AJ, et al: Validation of the Confusion Assessment Method for the Intensive Care Unit in older emergency department patients. Acad Emerg Med 2014; 21:180-187

27. Han JH, Vasilevskis EE, Schnelle JF, et al: The Diagnostic Performance of the Richmond Agitation Sedation Scale for Detecting Delirium in Older Emergency Department Patients. Acad Emerg Med 2015; 22:878-882

28. Hestermann U, Backenstrass M, Gekle I, et al: Validation of a German version of the Confusion Assessment Method for Delirium Detection in a sample of Acute Geriatric patients with High Prevalence of Dementia. Psychopathology 2009; 42:270-276

29. Koster S, Hensens AG, Oosterveld FG, et al: The delirium observation screening scale recognizes delirium early after cardiac surgery. Eur J Cardiovasc Nurs 2009; 8:309-314

30. Laurila JV, Pitkala KH, Strandberg TE, et al: Confusion Assessment Method in the diagnostics of delirium among aged hospital patients: Would it serve better in screening than as a diagnostic instrument? Int J Geriatr Psychiatry 2002; 17:1112-1119

31. Leung Jl, Leung Vc, Leung CM, et al: Clinical utility and validation of two instruments (the Confusion Assessment Method Algorithm and the Chinese version of Nursing Delirium Screening Scale) to detect delirium in geriatric inpatients. Gen Hosp Psychiatry 2008; 30:171-176 
32. Lin HS, Eeles E, Pandy S, et al: Screening in delirium: A pilot study of two screening tools, the Simple Query for Easy Evaluation of Consciousness and Simple Question in Delirium. Australas J Ageing 2015; 34:259-264

33. Luetz A, Heymann A, Radtke FM, et al: Different assessment tools for intensive care unit delirium: which score to use? Crit Care Med 2010; 38:409-418

34. Marcantonio ER, Ngo LH, O'Connor M, et al: 3D-CAM: derivation and validation of a 3-minute diagnostic interview for CAM-defined delirium: a cross-sectional diagnostic test study. Ann Intern Med 2014; 161:554-561

35. Martins S, Conceicao F, Paiva JA, et al: Delirium recognition by family: European Portuguese validation study of the family confusion assessment method. J Am Geriatr Soc 2014; 62:1748-1752

36. Martins S, Lourenco C, Pinto-de-Sousa J, et al: Validation study of the European Portuguese version of the Confusion Assessment Method (CAM). Int Psychogeriatr 2015; 27:777-784

37. Neelon VJ, Champagne MT, Carlson JR, et al: The NEECHAM Confusion Scale: construction, validation, and clinical testing. Nurs Res 1996; 45:324-330

38. Neufeld KJ, Leoutsakos JS, Sieber FE, et al: Evaluation of two delirium screening tools for detecting post-operative delirium in the elderly. Brit J Anaesth 2013; 111:612-618

39. Ni Chonchubhair A, Valacio R, Kelly J, et al: Use of the abbreviated mental test to detect postoperative delirium in elderly people. Br J Anaesth 1995; 75:481482

40. Pipanmekaporn T, Wongpakaran N, Mueankwan S, et al: Validity and reliability of the Thai version of the Confusion Assessment Method for the Intensive Care Unit (CAM-ICU). Clin Interv Aging 2014; 9:879-885

41. Pompei P, Foreman M, Cassel CK, et al: Detecting delirium among hospitalized older patients. Arch Intern Med 1995; 155:301-307

42. Powers JS, Doering T, Gordon S, et al: Exploring the utility of ultra-brief delirium assessments in a nonintensive care geriatric population: the GEM study. Gerontologist 2013; 53:1051-1055

43. Rhodius-Meester HFM, Campen van JPCM, Fung W, et al: Development and Validation of the Informant Assessment of Geriatric Delirium Scale (1-AGeD). Recognition of delirium in geriatric patients. Eur Geriatr Med 2013; 4:73-77

44. Rockwood K, Goodman J, Flynn M, et al: Cross-validation of the Delirium Rating Scale in Older Patients. J Am Geriatr Soc 1996; 44:839-842

45. Ryan K, Leonard M, Guerin S, et al: Validation of the confusion assessment method in the palliative care setting. Palliat Med 2009; 23:40-45

46. Salih SA, Paul S, Klein K, et al: Screening for delirium within the interRAI acute care assessment system. J Nutr Health Aging 2012; 16:695-700

47. Schuurmans MJ, Shortridge-Baggett LM,Duursma SA: The Delirium Observation Screening Scale: a screening instrument for delirium. Res Th Nurs Prac 2003; 17:31-50

48. Sörensen Duppils G,Johansson I: Predictive value and validation of the NEECHAM Confusion Scale using DSM-IV criteria for delirium as gold standard. Int J Older People Nurs 2011; 6:133-142

49. Thomas C, Kreisel SH, Oster P, et al: Diagnosing delirium in older hospitalized adults with dementia: Adapting the confusion assessment method to 
International Classification of Diseases, Tenth Revision, diagnostic criteria. J Am Geriatr Soc 2012; 60:1471-1477

50. van Gemert LA,Schuurmans MJ: The Neecham Confusion Scale and the Delirium Observation Screening Scale: capacity to discriminate and ease of use in clinical practice. BMC Nurs 2007; 6:1-3

51. Wongpakaran N, Wongpakaran T, Bookamana $\mathrm{P}$, et al: Diagnosing delirium in elderly Thai patients: utilization of the CAM algorithm. BMC Fam Prac 2011; 12:1-5

52. Monette J, Galbaud du Fort G, Fung SH, et al: Evaluation of the Confusion Assessment Method (CAM) as a screening tool for delirium in the emergency room. Gen Hosp Psychiatry 2001; 23:20-25

53. Mitasova A, Kostalova M, Bednarik J, et al: Poststroke delirium incidence and outcomes: validation of the Confusion Assessment Method for the Intensive Care Unit (CAM-ICU). Crit Care Med 2012; 40:484-490

54. Trzepacz PT, Baker RW,Greenhouse J: A symptom rating scale for delirium. Psychiatry Res 1988; 23:89-97 


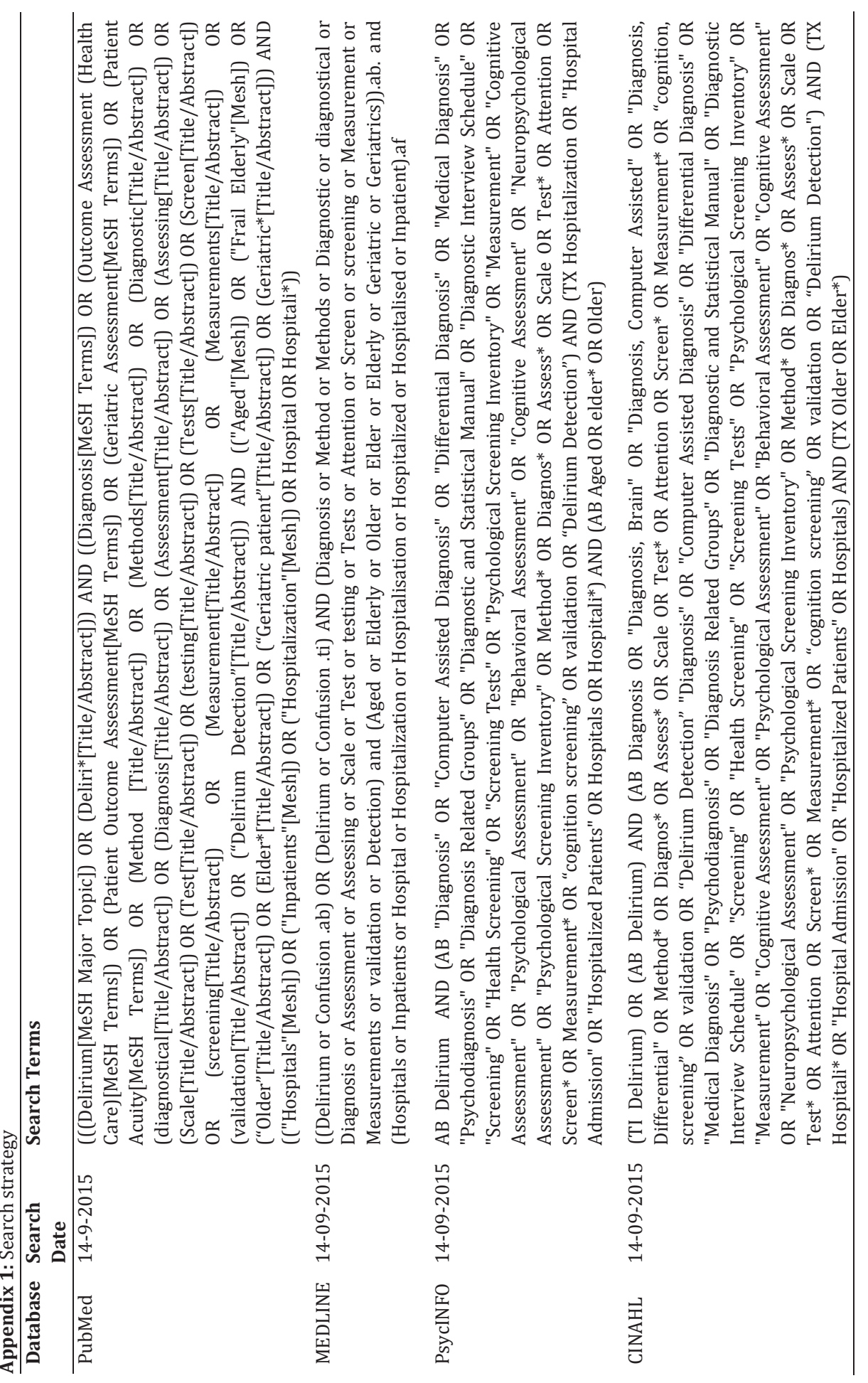


CHAPTER 2

Appendix 2: Risk of bias and applicability judgments (Quadas-2)

\section{Phase 1: State the review question:}

Patients (setting, intended use of the index test, presentation, prior testing):

Index tests:

Reference standard and target condition

Phase 2: Draw a flow diagram for the primary study

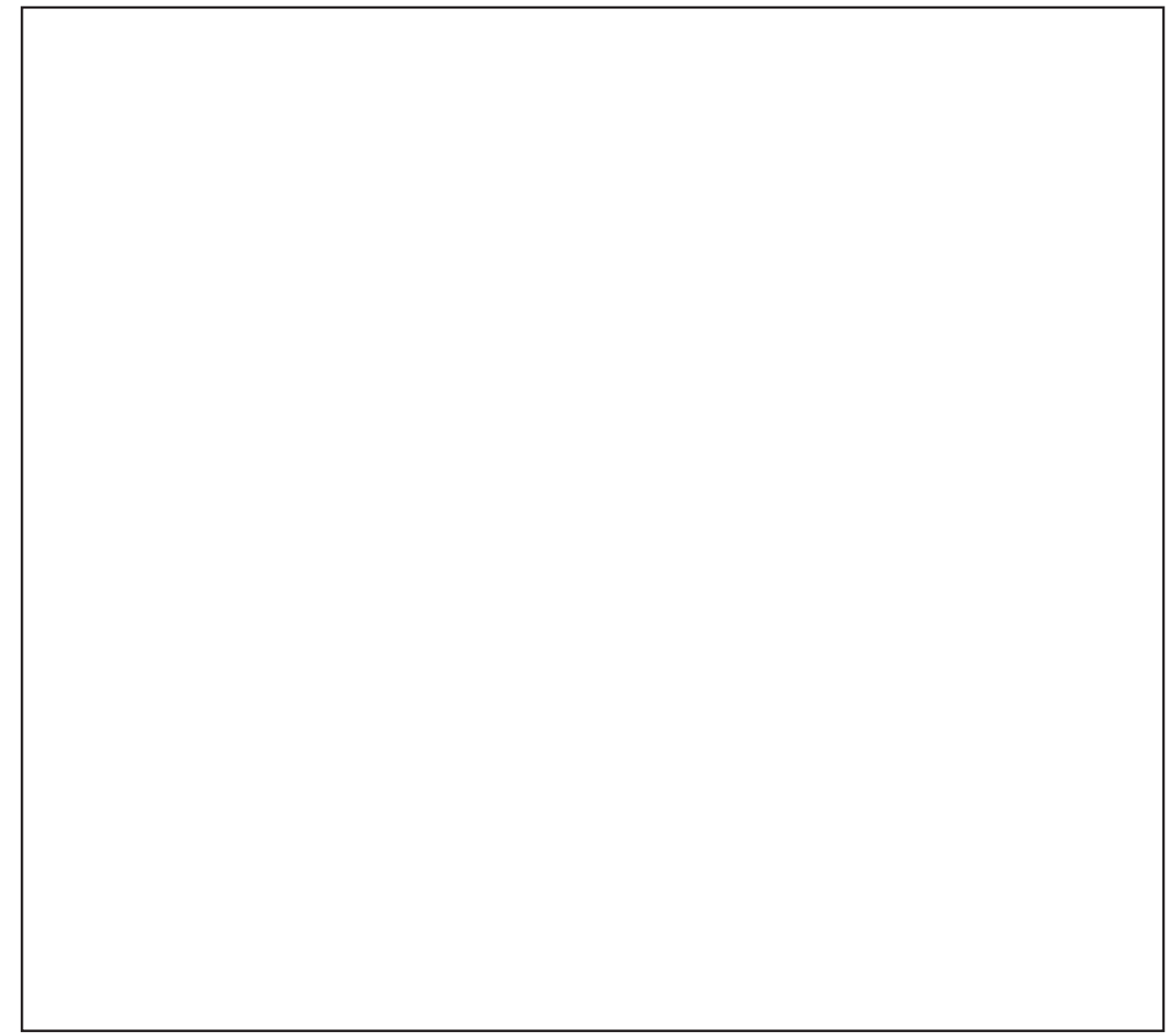




\begin{tabular}{|l|l|}
\hline Domain 1: Patient selection \\
\hline Describe methods of patient selection: \\
\hline Q1: Was a consecutive or random sample of patients enrolled? & Yes/No/Unclear \\
\hline Q2: Was a case-control design avoided? & Yes/No/Unclear \\
\hline Q3: Did the study avoid inappropriate exclusions? & Yes/No/Unclear \\
\hline Risk of bias: Could the selection of patients have introduced bias? & LOW/HIGH/UNCLEAR \\
\hline $\begin{array}{l}\text { Applicability: Is there concern that the included patients do not } \\
\text { match the review question? }\end{array}$ & LOW/HIGH/UNCLEAR \\
\hline
\end{tabular}

\begin{tabular}{|l|l|}
\hline Domain 2: Index test(s) (if more than 1 index test was used, please complete for each test) \\
\hline Describe the index test and how it was conducted and interpreted: \\
\hline $\begin{array}{l}\text { Q4: Were the index test results interpreted without knowledge of } \\
\text { the results of the reference standard? }\end{array}$ & Yes/No/Unclear \\
\hline $\begin{array}{l}\text { Q5: If a threshold was used, was it pre-specified? } \\
\text { Risk of bias: Could the conduct or interpretation of the index test } \\
\text { have introduced bias? }\end{array}$ & Yes/No/Unclear \\
\hline $\begin{array}{l}\text { Applicability: Is there concern that the index test, its conduct, or } \\
\text { interpretation differ from the review question? }\end{array}$ & LOW/HIGH/UNCLEAR \\
\hline
\end{tabular}




\begin{tabular}{|l|l|}
\hline \multicolumn{2}{|l|}{ Domain 3: Reference standard } \\
\hline \multicolumn{2}{|l|}{ Describe the reference standard and how it was conducted and interpreted: } \\
\hline $\begin{array}{l}\text { Q6: Is the reference standard likely to correctly classify the target } \\
\text { condition? }\end{array}$ & Yes/No/Unclear \\
\hline $\begin{array}{l}\text { Q7: Were the reference standard results interpreted without } \\
\text { knowledge of the results of the index test? }\end{array}$ & Yes/No/Unclear \\
\hline $\begin{array}{l}\text { Risk of bias: Could the reference standard, its conduct, or its } \\
\text { interpretation have introduced bias? }\end{array}$ & LOW/HIGH/UNCLEAR \\
\hline $\begin{array}{l}\text { Applicability: Is there concern that the target condition as defined by } \\
\text { the reference standard does not match the review question? }\end{array}$ & LOW/HIGH/UNCLEAR \\
\hline
\end{tabular}

\section{Domain 4: Flow and timing}

Describe any patients who did not receive the index test(s) and/or reference standard or who were excluded from the $2 \times 2$ table (refer to flow diagram):

Describe the time interval and any interventions between index test(s) and reference standard:

\begin{tabular}{|l|l|}
\hline $\begin{array}{l}\text { Q8: Was there an appropriate interval between index test(s) and } \\
\text { reference standard? }\end{array}$ & Yes/No/Unclear \\
\hline Q9: Did all patients receive a reference standard? & Yes/No/Unclear \\
\hline Q10: Did patients receive the same reference standard? & Yes/No/Unclear \\
\hline Q11: Were all patients included in the analysis? & Yes/No/Unclear \\
\hline Risk of bias: Could the patient flow have introduced bias? & LOW/HIGH/UNCLEAR \\
\hline
\end{tabular}



It is easy to be wise after the event.

- Sir A.C. Doyle (Sherlock Holmes, 1886)

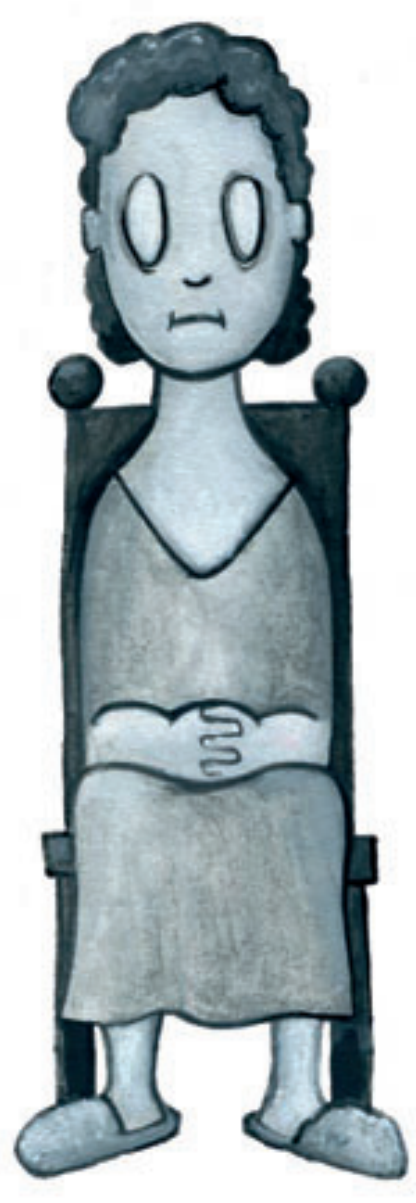


CHAPTER 3

Detection and management of hyperactive and hypoactive delirium in older patients during hospitalisation; a retrospective cohort study evaluating daily practice

This chapter was published as:

Eveline L van Velthuijsen, Sandra MG Zwakhalen, Wubbo J Mulder, Frans RJ Verhey and Gertrudis IJM Kempen. Detection and management of hyperactive and hypoactive delirium in older patients during hospitalisation: a retrospective cohort study evaluating daily practice. International Journal of Geriatric Psychiatry. 2017; doi: 10.1002/gps.4690 


\begin{abstract}
Objectives: To study daily hospital practice regarding detection and management and of hyperactive and hypoactive delirium of older patients during their hospitalisation.

Methods: A retrospective cohort study evaluating care as usual for older hospitalised patients with a delirium at the Maastricht University Medical Centre (MUMC+), a university hospital in the Netherlands. Inclusion criteria were older hospitalised patients (65+), diagnosed with delirium between January $1^{\text {st }}$ and December $31^{\text {st }}$ 2014. Data was retrieved from the patients' medical files. Delirium was categorised as hyperactive or hypoactive. Primary outcome measures were prevalence and management (pharmacological, reorientation, screening for delirium and delirium consultations, and physical restraints). Secondary outcomes were short-term adverse outcomes.

Results: Prevalence of delirium was 5\% (N=401), of which 77\% ( $n=307)$ was hyperactive and 23\% (n=94) was hypoactive. Significantly more patients with a hyperactive delirium received medication to manage the delirium than patients with a hypoactive delirium ( $89 \%$ versus $77 \%$ respectively, $\mathrm{p}=0.004)$. No other significant differences between the subtypes were found.

Conclusion: There was probably a strong under-recognition of delirium. Drugs were the main intervention of choice, especially for patients with a hyperactive delirium. The two subtypes did not differ on non-pharmacological management. The retrospective nature of this study sheds light on the status quo of recognition, management, and care as usual for the different delirium subtypes in daily hospital practice, which may help in forming new guidelines and protocols for the detection and treatment of delirium for older patients in hospitals.
\end{abstract}




\section{Introduction}

The number of older people admitted to hospitals has increased substantially over the past two decades. In 2010 more than half (55\%) of all people admitted to hospitals in the Netherlands were aged 65 years or over, compared to $31 \%$ in 1995. ${ }^{1}$ Age is an important risk factor for developing a delirium, and evidence suggests that between $29 \%$ and $64 \%$ of all older patients experience a delirium during hospitalisation. ${ }^{2}$ Delirium is found to be associated with many adverse outcomes, such as increased mortality rates, prolonged hospital stay, less and slower physical recovery, ${ }^{3}$ and increased risk of developing dementia, ${ }^{4,5}$ Despite this knowledge, delirium is often missed or misdiagnosed. ${ }^{6}$

Four different subtypes of delirium have been identified based on the motor symptoms exhibited by the patient: hyperactive, hypoactive, mixed, and without motor symptoms. ${ }^{7}$ Hyperactive delirium is characterised by (motor) agitation, restlessness, and sometimes aggressiveness. Hypoactive delirium is characterised by motor retardation, apathy, slowing of speech, and patients can appear to be sedated. ${ }^{8,9}$ Mixed delirium is a combination of hyperactive and hypoactive delirium. Delirium with no motor symptoms indicates that patients only experience cognitive symptoms of a delirium. The hypoactive subtype seems to be more common than the hyperactive subtype, $7,10,11$ though it is less likely to be discovered or reported, ${ }^{7}$ as these patients exhibit fewer behavioural problems and are often perceived as cooperative. ${ }^{12,13}$

The effects of the different subtypes on patient outcomes have been studied, but the results remain inconclusive. Some studies found that patient prognosis is worse after a delirium with hyperactive symptoms, ${ }^{14,15}$ while others found the prognosis to be poorer after a delirium with hypoactive symptoms. ${ }^{16,17}$ The hyperactive and hypoactive delirium also vary in the way they are managed: treatment with antipsychotic medication and the use of physical restraints is generally prompted by motor agitation and behavioural problems often present in patients with hyperactive delirium, ${ }^{18,19}$ whereas the use of antipsychotic medication in patients with the hypoactive subtype is generally avoided. ${ }^{20,21}$

Almost all of the abovementioned studies used a prospective study design; i.e. patients adhering to specific inclusion criteria were screened for delirium, and the required data was subsequently collected and analysed. A retrospective study design implies that the events being studied have already occurred, allowing researchers to study the status quo regarding recognition and management of delirium in daily, regular practice. However, it seems that only two studies used such a retrospective design to study the management of the different types of delirium among older people in a hospital setting, ${ }^{15,22}$ and neither studied adverse outcomes after discharge. Moreover, new Dutch hospital guidelines on how to diagnose and manage delirium in hospitalised adults were published in 2013, a year before the start of this study. This gives the authors the opportunity to see if implementing new guidelines improves the recognition and management of 
delirium, as we can compare our results to previous retrospective studies. The current retrospective cohort study therefore primarily aims to: 1 . analyse how often delirium in older patients, and specifically the hyperactive and hypoactive subtypes, are recognised and reported in daily hospital practice; 2. identify potential differences in management and care as usual between older hospitalised patients with a hyperactive or hypoactive delirium. As a secondary aim, the shortterm adverse outcomes of both patient groups are reported.

\section{Methods}

\section{Design}

A retrospective cohort study, analysing care as usual in daily hospital practice, was conducted at Maastricht University Medical Centre+ (MUMC+), a 715 bed university teaching hospital in the southern part of the Netherlands.

\section{Inclusion criteria and identification of the relevant patient files}

Patient files were included if: 1 . the patient was 65 years or older at the time of hospital admission; 2 . patients were admitted to the hospital between 1 January and 31 December 2014; 3. Patients were diagnosed with a delirium by a geriatrician, geriatric nurse practitioner (GNP) or a psychiatrist, or a delirium was mentioned in the patient's discharge letter; and 4. the patient consented to the use of his/her digital medical records for research.

Delirious patients were identified by going through the files of all older patients who had been looked at by a geriatrician, GNP or a psychiatrist during the study period. If a delirium was diagnosed and the patient adhered to the inclusion criteria, the patient file was included in the study. Additionally, all the discharge letters of patients 65 years or older were scanned for the presence of delirium using the words "delirium", "delier", "delirant", "verward" and "verwardheid" (Dutch for delirium, delirious, confused, and confusion, respectively), and for the ICD-10 codes of delirium: F050, F051, F058, and F059. If a patient was admitted to the hospital multiple times during the study period, only the first admission where the patient experienced a delirium was included in the study.

\section{Delirium subtypes}

At the MUMC+, delirium is divided into two subtypes: hyperactive, where patients experience motor agitation; and hypoactive, where patients experience motor retardation or only the cognitive symptoms without any motor symptoms. Symptoms of motor agitation include fidgeting, picking or pulling at medical equipment, and walking or wandering around the wards. For this study, patients with a mixed delirium are also considered to be hyperactive. The type of delirium is specified during the delirium consultation with the geriatrician, GNP or 
psychiatrist, based on clinical judgment and the Delirium Observation Screening Score (DOS) ${ }^{23}$ (See Table 1 for a description). In case a delirium was identified through the discharge letter, and no subtype was mentioned, the classification was made by author EvV based on the behaviour of the patient as described in the patient's file. The subtyping criteria were discussed with the GNPs and authors FRJV (head of the geriatric psychiatry department) and WJM (head of the geriatrics department) before the classification of the subtypes took place.

\section{Management of delirium}

The management of delirium in the MUMC+ can be either pharmacological or nonpharmacological. The main pharmacological treatment is the administration of haloperidol, though other antipsychotics or benzodiazepines are also sometimes used. Non-pharmacological management can be divided into three types: nursing interventions aimed at reorientation of the patient, psychosocial management, and physical restraint. Table 1 provides an overview and explanation of the nonpharmacological interventions.

Table 1. Non-pharmacological delirium management at the MUMC+, aimed at reorientation of the patient, and monitoring and managing the delirium

\begin{tabular}{|c|c|c|}
\hline Intervention & Type & Description \\
\hline Livingroom & Reorientation & $\begin{array}{l}\text { A living room for the older patients, run by volunteers } \\
\text { and an occupational therapist. The living room offers } \\
\text { interaction with other patients, a daily routine, and } \\
\text { activities such as music or art. }\end{array}$ \\
\hline Orientation box & Reorientation & $\begin{array}{l}\text { Contains a clock, calendar, diary, an information leaflet, } \\
\text { and a radio with CDs. }\end{array}$ \\
\hline Circadian rhythm & Reorientation & $\begin{array}{l}\text { To maintain a healthy sleep-wake cycle, or to avoid its } \\
\text { disruption. Physical therapists and the living room are } \\
\text { used to activate the patient during the day, and sedatives } \\
\text { in the morning are avoided where possible, and given in } \\
\text { the evening instead. }\end{array}$ \\
\hline Family participation & Reorientation & $\begin{array}{l}\text { Family have the opportunity to stay the night with the } \\
\text { patient, and to bring photos, pillows, and bedsheets from } \\
\text { home to make the patient feel more at ease in the } \\
\text { hospital. }\end{array}$ \\
\hline Delirium Consultation & Psychosocial & $\begin{array}{l}\text { A consultation done by a nurse practitioner specialised in } \\
\text { delirium, a geriatrician, or by a psychiatrist. If a patient is } \\
\text { diagnosed with delirium, advice is given on the best } \\
\text { treatment and interventions. }\end{array}$ \\
\hline Screening & Psychosocial & $\begin{array}{l}\text { The Delirium Observation Screening Score }{ }^{23} \text { is used to } \\
\text { screen for delirium and measuring delirium severity. It } \\
\text { consists of } 13 \text { observations which can be scored as } \\
\text { present ( } 1 \text { point) or not present ( } 0 \text { points). The maximum } \\
\text { amount of points is } 13 \text {, and the cut-off score is three. It is } \\
\text { administered three times a day: during the morning, day } \\
\text { and evening nursing shifts. }\end{array}$ \\
\hline Physical Restraints & Restraint & $\begin{array}{l}\text { Physical restraints are used to prevent a patient from } \\
\text { harming themselves or others. The main mode of } \\
\text { restraining is an enclosed bed canopy system. }\end{array}$ \\
\hline
\end{tabular}




\section{Data extraction}

The following data was extracted from the digital patient files by author EvV:

Demographic and baseline data: age at hospital admission, sex, living conditions prior to hospital admission (i.e. was the patient living at home or in a nursing home), reason for admission, comorbidities, presence of dementia, number and type of medications used at time of delirium diagnosis, ward of admission and ward where the delirium had been diagnosed.

Information related to the delirious episode: duration and cause of the delirium. The duration was measured from the date on which the diagnosis of delirium was first confirmed in the digital patient file, till, in order of importance: 1. an MD or a GNP noted in the patient files that the delirium was in remission or had passed; 2 . pharmacological treatment for the delirium was ceased because of abating symptoms; 3. the DOS remained below three points for three consecutive measurements; 4 . the patient had died during hospital admission; or 5. the patient had been discharged from the hospital.

Delirium management: The pharmacological, non-pharmacological, and psychosocial management of delirium (see Table 1).

Adverse outcomes: length of hospital stay, mortality during hospital stay, and discharge destination (back home, to a nursing home, or to a rehabilitation facility). Discharge destination was only measured for those patients who were living at their own home prior to admission, as patients living in a nursing home are always discharged back to the nursing home.

\section{Ethics \& consent}

The study was approved by the Medical Ethics Committee of Maastricht University and MUMC+ (project number 144169), and by the board of directors of MUMC+.

\section{Statistical methods}

Differences between groups for baseline characteristics were calculated using a two-tailed independent samples t-test for age, number of medications used, and number of comorbidities. A chi $^{2}$ was used for sex, living conditions prior to admission, and presence of dementia. Differences in management and discharge destination between the delirium subtypes were tested using logistic regression analyses. A generalised linear mixed regression on a negative binomial distribution was performed to examine the relationship between type of delirium on the length of stay and duration of the delirium, while controlling for age, sex, presence of dementia, and hospital ward where the patient was admitted when the delirium was diagnosed. Due to the skewed distribution of length of stay and duration of the delirium, the mode and range were given for these outcomes, instead of the mean and standard deviation. A conservative cut-off of $p \leq 0.01$ was chosen to minimise the chance of a type I error after multiple testing, with a confidence interval of $99 \%$. 
Missing data were defined as such, and were not taken into account in the analyses. Data were analysed using SPSS version 22 (IBM Corp., Armonk, NY).

\section{Results}

\section{Delirium recognition and subtypes}

No patients objected to the use of their patient files.

Between January 12014 and December 31 2014, a total of 7.907 patients aged 65 years or older were admitted to the MUMC+. Delirium was confirmed in 401 older patients (5\%); 307 (77\%) were of the hyperactive subtype, and 94 (23\%) were of the hypoactive subtype.

\section{Sample characteristics}

Patient files $(\mathrm{N}=401)$ were identified through the logs of the psychiatry and geriatric wards $(n=267)$ and through scanning discharge letters $(n=274) ; 140$ patients were identified through both pathways. Patients who suffered from a hyperactive delirium did not differ significantly from patients who suffered a hypoactive delirium on any of the baseline characteristics. An overview of the baseline characteristics for the total group and per subtype is presented in Table 2.The main reasons for hospital admission were cardiovascular problems (20\%; $n=79)$, infections (19\%; $n=75)$, and hip or femur fractures $(15 \% ; n=61)$. Twenty four people $(6 \%)$ were admitted because of a delirium or confusion. Table 3 presents an overview of the reasons for admission, and classification of the individual problems into the different categories can be found in Appendix A. Most patients were admitted through the surgical wards (including the cardio-thoracic surgery ward) (15\%; $n=61)$, through the emergency department (13\%; $n=52)$, and through the department of internal medicine (13\%; $n=51)$.

Table 2. Demographic characteristics and baseline data of the total sample and of hyperactive and hypoactive subgroups.

\begin{tabular}{lllll} 
& $\begin{array}{l}\text { Total } \\
\mathbf{N = 4 0 1}\end{array}$ & $\begin{array}{l}\text { Hyperactive } \\
\mathbf{N = 3 0 7}(\mathbf{7 7 \%} \%\end{array}$ & $\begin{array}{l}\text { Hypoactive } \\
\mathbf{N = 9 4}(\mathbf{2 3} \%)\end{array}$ & $\mathbf{p}$-value \\
\hline Female n (\%) & $167(42 \%)$ & $122(40 \%)$ & $45(48 \%)$ & $\mathrm{p}=0.16$ \\
Age m \pm sd (range) & $81 \pm 7(65-99)$ & $81 \pm 7(65-99)$ & $80 \pm 7(65-93)$ & $\mathrm{p}=0.49$ \\
Living at home before admission $\mathrm{n}(\%)$ & $314(78 \%)$ & $243(79 \%)$ & $71(76 \%)$ & $\mathrm{p}=0.46$ \\
Presence of dementia n (\%) & $96(24 \%)$ & $71(23 \%)$ & $25(27 \%)$ & $\mathrm{p}=0.49$ \\
\#Comorbidities m \pm sd (range) & $4 \pm 2(1-12)$ & $4 \pm 2(1-12)$ & $4 \pm 2(1-9)$ & $\mathrm{p}=0.75$ \\
\#Medications m \pm sd (range) & $8 \pm 4(0-20)$ & $8 \pm 4(0-20)$ & $8 \pm 4(0-19)$ & $\mathrm{p}=0.59$ \\
\hline
\end{tabular}

A $\chi^{2}$ was used to check for statistical differences between the subtypes for sex, living at home before admission, and presence of dementia. An independent samples t-test was used to check for statistical differences between the subtypes on number of comorbidities and number of medications used 
Table 3. Primary reasons for admission of the patient cohort

\begin{tabular}{ll}
\hline${\text { Reasons for } \text { admission }^{\mathbf{a}}}$ & Total (N=401) \\
\hline Cardiovascular problems & $79(20 \%)$ \\
Infections & $75(19 \%)$ \\
Hip or femur fractures & $61(15 \%)$ \\
General downturn/decay & $32(8 \%)$ \\
Oncological causes & $29(7 \%)$ \\
CVA/trauma capitis & $27(7 \%)$ \\
Delirium or confusion & $24(6 \%)$ \\
Pulmonary causes (other than infections) & $23(6 \%)$ \\
Gastro-intestinal and intra-abdominal issues & $23(6 \%)$ \\
Other & $28(7 \%)$
\end{tabular}

a Reasons for admission have been classified into the categories as mentioned in the table. The individual reasons for admission in each category can be found in Appendix A.

The most commonly reported cause of delirium was an infection (urinary tract infection, pneumonia, or other infections) (38\%; $n=154)$ and surgery $(24 \% ; n=95)$. Other causes of delirium were medication use $(5 \% ; n=19)$, and falls $(3 \% ; n=14)$. For the remaining patients $(30 \% ; n=119)$, no direct cause of the delirium could be established.

\section{Delirium management}

Most patients (86\%; $n=346)$ received medication (mainly Haloperidol) to manage their delirium. Patients with a hyperactive delirium received medication significantly more often than patients with a hypoactive delirium (89\% versus $77 \%$ respectively; $\mathrm{p}=.004)$. There were no significant differences between the groups on any of the other interventions. The results of the regression models for the effect of delirium type on used interventions can be found in Table 4.

\section{Adverse outcomes}

Table 5 describes the length of delirium, length of stay, in-hospital mortality, and discharge destination for the patients included in this study. Less than half (47\%) of the patients from our sample could return back home after the hospital stay, and 15\% died during hospitalisation. 
Table 4. Pharmacological, non-pharmacological, and psychosocial interventions which were employed for managing the delirium subtypes

\begin{tabular}{|c|c|c|c|c|c|c|c|}
\hline \multirow{2}{*}{$\begin{array}{l}\text { Management } \\
\text { type }\end{array}$} & \multirow{2}{*}{$\begin{array}{l}\text { Total } \\
(N=401)\end{array}$} & \multirow{2}{*}{$\begin{array}{l}\text { Hyperactive } \\
(\mathrm{N}=307)\end{array}$} & \multirow{2}{*}{$\begin{array}{l}\text { Hypoactive } \\
(\mathrm{N}=94)\end{array}$} & \multirow[t]{2}{*}{ OR } & \multicolumn{2}{|c|}{$99 \% \mathrm{CI}^{\mathrm{a}}$} & \multirow[t]{2}{*}{ p-value ${ }^{a}$} \\
\hline & & & & & Lower & upper & \\
\hline Medication & $346(86 \%)$ & $274(89 \%)$ & $72(77 \%)$ & 2.44 & 1.09 & 5.45 & $\mathrm{p}=.004$ \\
\hline DOS-scores & $300(75 \%)$ & $232(76 \%)$ & $68(72 \%)$ & 1.23 & 0.59 & 2.56 & $\mathrm{p}=.47$ \\
\hline Consultation & 267 (67\%) & $210(68 \%)$ & 57 (61\%) & 1.46 & 0.74 & 2.88 & $\mathrm{p}=.15$ \\
\hline Reorientation ${ }^{b}$ & 278 (69\%) & 213 (69\%) & $65(69 \%)$ & 1.07 & 0.52 & 2.21 & $\mathrm{p}=.81$ \\
\hline Physical restraint & $121(30 \%)$ & $101(33 \%)$ & $20(21 \%)$ & 1.86 & 0.87 & 3.96 & $\mathrm{p}=.03$ \\
\hline Combined $^{c}$ & $239(60 \%)$ & $189(62 \%)$ & $50(53 \%)$ & 1.47 & 0.76 & 2.85 & $\mathrm{p}=.13$ \\
\hline No interventions & $16(4 \%)$ & $9(3 \%)$ & $7(7 \%)$ & 0.28 & 0.06 & 1.20 & $\mathrm{p}=.02$ \\
\hline
\end{tabular}

Differences in management between the subtypes were calculated using a logistic regression. The model was corrected for age, sex, dementia, length of delirium, and ward where the delirium was diagnosed. $\mathrm{OR}=$ odds ratio, $\mathrm{CI}=$ confidence interval

a Statistical significance levels are set at 0.01 to minimise the chance of a type 1 error after multiple testing and confidence intervals at $99 \%$.

${ }^{\mathrm{b}}$ See Table 1 for interventions aimed at reorientation.

c The row "combined": these patients received both pharmacological and non-pharmacological interventions during the delirious episode. There is overlap between the number is this row and those in the row "medication" and "reorientation".

Table 5. Short-term adverse outcomes for all patients with a delirium, and according to subtype.

\begin{tabular}{|c|c|c|c|c|c|c|c|}
\hline \multirow{2}{*}{$\begin{array}{l}\text { Adverse } \\
\text { outcomes }\end{array}$} & \multirow{2}{*}{$\begin{array}{l}\text { Total } \\
(\mathrm{N}=401)\end{array}$} & \multirow{2}{*}{$\begin{array}{l}\text { Hyperactive } \\
(\mathrm{N}=307)\end{array}$} & \multirow{2}{*}{$\begin{array}{l}\text { Hypoactive } \\
(\mathrm{N}=94)\end{array}$} & \multirow{2}{*}{$\begin{array}{l}\text { B or } \\
\text { OR }^{\text {a }}\end{array}$} & \multicolumn{2}{|c|}{ 99\% CI } & \multirow[t]{2}{*}{ p-value ${ }^{b}$} \\
\hline & & & & & lower & upper & \\
\hline LoS m (range) & $8(1-160)$ & 7 (1-126) & $8(2-160)$ & $B=3.44$ & 1.85 & 5.04 & $\mathrm{p}=.58$ \\
\hline LoD m (range) & $3(1-99)$ & $3(1-91)$ & $6(1-99)$ & $B=0.02$ & -0.35 & 0.31 & $\mathrm{p}=.88$ \\
\hline $\begin{array}{l}\text { Discharged } \\
\text { homec }^{c}, \mathrm{n}\end{array}$ & $124(47 \%)$ & 97 (46\%) & 27 (51\%) & $\mathrm{OR}=0.80$ & 0.33 & 1.92 & $\mathrm{p}=.51$ \\
\hline Died $^{\mathrm{d}}, \mathrm{n}$ & 59 (15\%) & 38 (12\%) & $21(22 \%)$ & $\mathrm{OR}=0.45$ & 0.20 & 1.02 & $\mathrm{p}=.012$ \\
\hline
\end{tabular}

A generalised linear mixed negative binomial regression was used to check the differences on length of stay and length of delirium between the subtypes, and a logistic regression was used for "discharged back home" and "died in hospital". OR=odds ratio, CI=confidence interval, m=mode, LoS=Length of hospital stay measured in days, LoD=Length of delirium measured in days.

a $\mathrm{B}$ is the coefficient provided for generalised linear mixed negative binomial regressions, and Odds Ratio (OR) is provided for logistic regression.

b Statistical significance levels are set at 0.01 , and confidence intervals at $99 \%$ to minimise the chance of a type I error after multiple testing.

${ }^{c} \mathrm{~N}=265$ because only people who were living in their own home before admission, and did not die during hospital stay were taken into account for this outcome.

The model was corrected for age, sex, dementia, length of delirium, and ward where the delirium was diagnosed.

${ }^{\mathrm{d}}$ Only patients who died during their hospital stay. 


\section{Discussion}

In this retrospective cohort study, we examined the differences in management and in short and long-term adverse outcomes between hyperactive and hypoactive subtypes of delirium among older hospitalised patients. Significantly more patients with a hyperactive delirium received antipsychotic medication (Haloperidol or other) compared to patients with a hypoactive delirium. No significant differences were found for any of the other interventions or adverse outcomes, though a trend was found for higher in-hospital mortality among patients with a hypoactive delirium.

Previous retrospective studies found a prevalence rate of delirium of $2 \%$ in an Irish hospital ${ }^{22}$ and $2,8 \%$ in a US hospital setting. ${ }^{24}$ However, prospective studies showed that the prevalence of delirium in older hospitalised patients varies from $29 \%$ to $64 \%$, depending on the hospital ward. ${ }^{2}$ Our results indicate that there is probably a substantial under-recognition and/or underreporting of delirium in the MUMC+, despite new Dutch guidelines for the recognition and treatment of delirium in older hospitalised patients being published in 2013.25 Moreover, only 25\% of the identified patients with delirium in our study were classified as being of the hypoactive subtype, even though various studies have found that the hypoactive subtype is the most prevalent one with prevalence rates up to $56 \%{ }^{7,10,11}$ The retrospective nature of this study reflecting regular, daily practice is probably the reason for this under-recognition. Medical staff may often overlook patients with a hypoactive delirium because they are mostly passive and quiet, and are perceived as cooperative. ${ }^{12,13}$ Furthermore, delirium is considered by many physicians to be a harmless side-effect of hospitalisation. ${ }^{26}$ This could mean that for some patients the delirium, and especially the hypoactive subtype, may have been recognised by a nurse or physician, but not reported in the patient file or discharge letter, and subsequently, not included in this study.

Medication, mostly Haloperidol, was preferred over non-pharmacological interventions for the management of both delirium subtypes, despite the guidelines advocating the use of non-pharmacological interventions before resorting to medication. ${ }^{25,27}$ Almost $90 \%$ of the patients with a hyperactive delirium, and $77 \%$ of the patients with a hypoactive delirium received antipsychotic medication to treat the delirium. In particular, the high percentage of patients with a hypoactive delirium receiving medication is noticeable, as the NICE and Dutch guidelines advise physicians to be sparing in the prescription of antipsychotics for the treatment of these patients. ${ }^{25,27}$ Moreover, two recent systematic reviews have concluded that there is little evidence for the efficacy of antipsychotics in the treatment of delirium, and that the available evidence is generally weak or circumstantial.28,29 In addition, the use of antipsychotics in older patients, in particular those with dementia, is a cause for concern, as they can increase the risk of mortality. ${ }^{30,31}$ Non-pharmacological interventions, aimed at reorientation, however, have been proven to be (cost)effective in many different international 
studies, ${ }^{32}$ and should - in accordance with international guidelines $-25,27$ be considered first, followed by medication only if non-pharmacological management seems insufficient. Also, just two thirds of the delirious patients were referred to a geriatric consultation liaison, even though their expertise can provide nurses and physicians with the necessary information and tools to adequately detect and manage delirium.

The two patient groups in our cohort did not differ significantly on any of the adverse outcomes, though in-hospital mortality had a tendency to be higher among patients with a hypoactive delirium compared to patients with a hyperactive delirium. Previous studies found that patients with a hypoactive delirium had a longer length of hospital stay and higher mortality rates compared to patients with a hyperactive delirium. ${ }^{16,17,19}$ One study reported higher mortality rates for patients with a hyperactive delirium, and also found that these patients were more likely to be admitted to a nursing home after discharge. ${ }^{14}$ However, in light of the low overall prevalence of delirium, and the high relative prevalence of the hyperactive subtype, results on adverse outcomes should be interpreted with caution.

\section{Strengths and limitations}

The retrospective, descriptive nature of this study has both strengths and limitations. It let us examine how often delirium is reported, and what the care as usual is in a regular hospital setting. In addition, prospective studies can be difficult to perform as the temporary or permanent decrease of mental competence resulting from the delirious episode makes it more difficult to receive informed consent from the patients. However, there is a bias in the retrospective design, as especially patients with a more severe or hyperactive delirium may have been recognised or reported, thus excluding patients with a less severe or hypoactive delirium. Also, in this study we were not able to differentiate between the hyperactive and mixed subtype, as in the MUMC+ a delirium is classified as being either with motor agitation (hyperactive) or without motor agitation (hypoactive). As such, all patients experiencing a delirium with motor agitation were classified as being hyperactive, and all patients experiencing a delirium with motor retardation or without any motor symptoms were classified as being hypoactive. This may have affected our results, as the contrast between the two subtypes may have become less. Lastly, the low prevalence of delirium in this study, and the relatively high prevalence of the hyperactive subtype may have affected the adverse outcome results. Therefore, no final conclusions can be made from the adverse outcome results, and these should be interpreted with caution. Retrospective designs, however, also have a considerable strength: they enable us to study the situation as it is in reality, without the inherent focus on delirium of a prospective design. This study, therefore, has shed light on the current recognition rates and practices regarding delirium management, which is vital information for the development of guidelines and plans for improving delirium care. 


\section{Conclusions}

Our findings indicate that delirium in older hospitalised patients is probably substantially under-recognised and/or under-reported, particularly the hypoactive subtype, despite the introduction of new guidelines on delirium in hospitalised adults. Furthermore, almost $90 \%$ of all delirious patients received medication. Considering the weak or circumstantial evidence for pharmacological treatment of delirium, and the wealth of evidence in favour of non-pharmacological interventions aimed at reorientation, the latter need to be used more often, and should be promoted among nursing staff. Also, physicians and nurses should not only be taught how to recognise and manage a delirium, but they should be made aware of the different subtypes and their corresponding treatments, the severe adverse effects of a delirium, and the (unnecessary) high costs involved with this disorder. Considering the wealth of evidence on the negative effects of delirium and the availability of hospital guidelines on the detection and management of delirium, it is difficult to understand that delirium in older hospital patients is still so strongly under-recognised. Future research should focus on the differences between the delirium subtypes, the underlying causes, aetiology, and strategies to improve detection, prevention and treatment of delirium by the medical staff. 


\section{References}

1. Central Agency for Statistics (CBS): Gezondheid en zorg in cijfers, Den Haag, Centraal Bureau voor de Statistiek, 2015

2. Inouye SK, Westendorp RGJ,Saczynski JS: Delirium in elderly people. Lancet 2014; 383:911-922

3. Witlox J, Eurelings LS, de Jonghe JF, et al: Delirium in elderly patients and the risk of postdischarge mortality, institutionalization, and dementia: a metaanalysis. JAMA 2010; 304:443-451

4. Davis DH, Muniz Terrera G, Keage H, et al: Delirium is a strong risk factor for dementia in the oldest-old: a population-based cohort study. Brain 2012; 135:2809-2816

5. Krogseth M, Watne LO, Juliebo V, et al: Delirium is a risk factor for further cognitive decline in cognitively impaired hip fracture patients. Arch Gerontol Geriatr 2016; 64:38-44

6. Siddiqi N, House A0,Holmes JD: Occurrence and outcome of delirium in medical in-patients: a systematic literature review. Age ageing 2006; 35:350364

7. Albrecht JS, Marcantonio ER, Roffey DM, et al: Stability of Postoperative Delirium Psychomotor Subtypes in Individuals with Hip Fracture. J Am Geriatr Soc 2015; 65:970-976

8. Lipowski ZJ: Transient cognitive disorders (delirium, acute confusional states) in the elderly. Am J Psychiat 1983; 140:1426-1436

9. Meagher DJ: Motor subtypes of delirium: past, present and future. Int Rev Psychiatr 2009; 21:59-73

10. Boettger S,Breitbart W: Phenomenology of the subtypes of delirium: phenomenological differences between hyperactive and hypoactive delirium. Palliat Support Care 2011; 9:129-135

11. Meagher DJ, Leonard M, Donnelly S, et al: A longitudinal study of motor subtypes in delirium: frequency and stability during episodes. J Psychosom Res 2012; 72:236-241

12. Inouye SK, Foreman MD, Mion LC, et al: Nurses' recognition of delirium and its symptoms: comparison of nurse and researcher ratings. Arch Intern Med 2001; 161:2467-2473

13. Rice KL, Bennett M, Gomez M, et al: Nurses' recognition of delirium in the hospitalized older adult. Clin Nurse Spec 2011; 25:299-311

14. Marcantonio E, Ta T, Duthie E, et al: Delirium severity and psychomotor types: their relationship with outcomes after hip fracture repair. J Am Geriatr Soc 2002; 50:850-857

15. Kobayashi K, Takeuchi 0 , Suzuki M, et al: A retrospective study on delirium type. Jpn J Psychiatry Neurol 1992; 46:911-917

16. Meagher DJ, Leonard M, Donnelly S, et al: A longitudinal study of motor subtypes in delirium: relationship with other phenomenology, etiology, medication exposure and prognosis. J Psychosom Res 2011; 71:395-403

17. Robinson TN, Raeburn CD, Tran ZV, et al: Motor subtypes of postoperative delirium in older adults. Arch Surg 2011; 146:295-300 
18. Freeman S, Hallett C, McHugh G: Physical restraint: experiences, attitudes and opinions of adult intensive care unit nurses. Nurs Crit Care 2015;

19. O'Keeffe ST, Lavan JN: Clinical significance of delirium subtypes in older people. Age Ageing 1999; 28:115-119

20. Inouye SK: Delirium in older persons. New Engl J Med 2006; 354:1157-1165

21. British Geriatrics Society and Royal College of Physicians: Guidelines for the prevention, diagnosis and management of delirium in older people in hospital, 2006

22. Rooney S, Qadir M, Adamis D, et al: Diagnostic and treatment practices of delirium in a general hospital. Aging Clin Exp Res 2014; 26:625-633

23. Schuurmans MJ, Shortridge-Baggett LM, Duursma SA: The Delirium Observation Screening Scale: a screening instrument for delirium. Res Theory Nurs Pract 2003; 17:31-50

24. McCoy TH Jr., Snapper L, Stern TA, et al: Underreporting of Delirium in Statewide Claims Data: Implications for Clinical Care and Predictive Modeling. Psychosomatics 2016;

25. Nederlandse Vereniging voor Klinische Geriatrie: Richtlijn Delier Volwassenen. 2013;

26. Leslie DL, Inouye SK: The importance of delirium: economic and societal costs. J Am Geriatr Soc 2011; 59:S241-S243

27. National Institute for Care and Health Excellence: Delirium: prevention, diagnosis and management (NICE guideline CG103), 2010

28. Schrijver EJ, de Graaf K, de Vries OJ, et al: Efficacy and safety of haloperidol for in-hospital delirium prevention and treatment: A systematic review of current evidence. Eur J Intern Med 2016; 27:14-23

29. Neufeld KJ, Yue J, Robinson TN, et al: Antipsychotic Medication for Prevention and Treatment of Delirium in Hospitalized Adults: A Systematic Review and Meta-Analysis. J Am Geriatr Soc 2016;

30. Jeste DV, Blazer D, Casey D, et al: ACNP White Paper: update on use of antipsychotic drugs in elderly persons with dementia. Neuropsychopharmacol 2008; 33:957-970

31. Schneider LS, Dagerman KS, Insel P: Risk of death with atypical antipsychotic drug treatment for dementia: meta-analysis of randomized placebo-controlled trials. J Am Med Assoc 2005; 294:1934-1943

32. Inouye S, Robinson T, Blaum C: Postoperative delirium in older adults: best practice statement from the American Geriatrics Society. J Am Coll Surg 2015; 220:136-148 e131 

Frisse beweging in de buitenlucht, zei die dokter tegen me.

Ach, wat weet zo'n arts

van de tere gezondheid van een heer?

- Marten Toonder (Olivier B. Bommel, 1973)

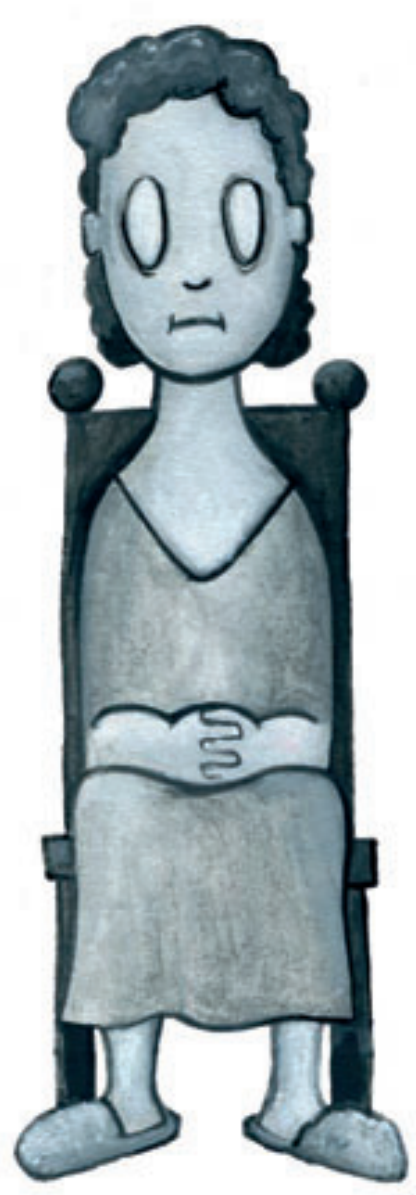




\section{CHAPTER 4 Effects of a medication review on delirium in older hospitalised patients: a comparative retrospective cohort study}

This chapter has been published as:

Eveline L van Velthuijsen, Sandra MG Zwakhalen, Evelien Pijpers, Liesbeth I van de Ven, Ton Ambergen, Wubbo J Mulder, Frans RJ Verhey and Gertrudis IJM Kempen. Effects of a medication review on delirium in older hospitalised patients: a comparative retrospective cohort study. Drugs \& Aging. 2018;35(2):153-161 
Abstract

Background: Delirium in older hospitalised patients is a common and serious disorder. Polypharmacy and certain medications are risk factors for developing a delirium. A medication review could benefit delirious older hospitalised patients.

Objectives: 1. Evaluate the effects of the review on length of delirium, length of hospital stay, mortality, and discharge destination; and 2. Describe and analyse the proposed changes to medication and its implementation by the treating physician. Setting: Maastricht University Medical Centre+.

Methods: We compared two cohorts of older patients with delirium: the first cohort from before introducing the medication review, and a second cohort five months after introduction of the medication review. Data was extracted from the patients' digital medical records.

Results: A significant interaction effect of cohort and number of medications taken by the patient was found for duration of delirium: patients from the second cohort taking between zero and six medications had significantly shorter delirious episodes than patients in the first cohort. This effect bordered on significance for patients taking between seven and 11 medications, but disappeared for patients taking 12 or more medications. No other statistically significant differences were found between the cohorts. The proposed changes in medication were implemented for $71 \%$ of the patients.

Conclusion: A medication review seems to significantly decrease the length of an older patient's delirious episode. Given the clinical relevance of these findings, we advise medication reviews for all older patients who are delirious or are at risk of developing a delirium. 


\section{Introduction}

Delirium is a serious neuropsychiatric syndrome characterised by an acute decline of cognitive functions and disorientation ${ }^{1}$ affecting between 29 and $64 \%$ of older hospitalised patients. ${ }^{2}$ It is associated with many negative outcomes, including increased mortality rates, prolonged hospital stay, decreased physical recovery, and higher rates of institutionalisation. ${ }^{3-5}$ These negative consequences not only affect patients, but also greatly increase healthcare costs. ${ }^{6-9}$ There is substantial evidence suggesting that delirium is preventable in $30 \%$ to $40 \%$ of cases, mainly by avoiding iatrogenic risk factors which could trigger a delirium, such as immobility, dehydration, malnutrition, infections (e.g. urinary tract infection as a result of an unnecessary urinary catheter) and polypharmacy. ${ }^{10,11}$

One method of reducing delirium induced by iatrogenic risk factors is the creation of a multidisciplinary geriatric team, which focuses on optimising care for frail older patients at risk for developing a delirium. These teams, often focussing on risk factors such as immobility, dehydration, malnutrition and pain, have been found to be effective in reducing delirium incidence and severity in previous studies. ${ }^{11-16}$ Additionally, non-pharmacological interventions can be used to prevent delirium, such as the use of a clock and calendar for orientation, bringing pictures from home to provide a point of recognition for a patient and making sure patients use their visual and/or hearing aids adequately. ${ }^{2}$ However, these multicomponent deliriumprevention groups are often nurse-led and do not focus specifically on medicationrelated problems, such as polypharmacy, unnecessary medications, or harmful medication-interactions, even though polypharmacy is one of the main iatrogenic risk factors for delirium in older hospitalised patients. ${ }^{17}$

Moreover, the evaluation of these teams has always been conducted under controlled conditions in a research setting, and not in a daily-practice setting. The aims of the present study, therefore, are 1. to describe the type of changes in medication proposed as a result of the medication review, and analyse the implementation of these proposed changes by the treating physician; and 2 . to evaluate the benefits of the medication review on the length of delirium, length of hospital stay, in-hospital mortality, and discharge destination in older hospitalised patients who suffered a delirium. We hypothesise that patients who received a medication review, and especially those patients with polypharmacy, will benefit the most, resulting in shorter duration of delirium and length of stay, that there will be less in-hospital mortality and that patients will be discharged back home instead of to a nursing home or alternative living environment more often compared to peers who did not receive a medication review. 


\section{Methods}

\section{Design}

A retrospective comparative cohort study using information from digital medical records was conducted at the Maastricht University Medical Centre+ (MUMC+), a 715 bed university teaching hospital in the south of the Netherlands.

\section{The medication review process}

The MUMC+ does not have a separate geriatric department, but geriatricians and geriatric nurse practitioners (GNPs) operate throughout the hospital in a liaisonconsultation role for older patients (aged 70 years or over). Since 2014, all older patients admitted to the MUMC+ are screened for frailty using the Maastricht Frailty Screening Tool for Hospitalised Patients (MFST-HP). ${ }^{18}$ Patients with a score of eight or more on the MFST-HP are consulted by a GNP. Additionally, a consultation from a geriatrician or GNP can be requested by a nurse or physician. During these consultations delirium risk is assessed and preventive measures are discussed with the nursing staff of the patient's ward. If a patient is delirious at the time of the consultation, delirium management is discussed with the nursing staff. In addition to these consultations, a weekly geriatric meeting was set up in February 2015, consisting of a geriatrician, a geriatrician in training, two GNPs, and a clinical pharmacist. The goal of this additional geriatric meeting was to provide a medication review for all patients who received a geriatric consult. Every week, one day before the weekly geriatric meeting, the clinical pharmacist (author LvdV) receives a list of all the patients who received a consultation from the geriatrician or GNP and were still hospitalised at time of the weekly geriatric meeting. The clinical pharmacist then uses the STOPP (Screening Tool of Older Person's Prescriptions) and START (Screening Tool to Alert doctors to Right Treatment) criteria ${ }^{19}$ to assess the indication, necessity and adequacy of every medication on a patient's medication list, taking into account age and comorbidities of the patient, as well as interactions with other medications and possible negative side effects of the medication. During the geriatric meeting the clinical pharmacist discusses the medication review with the geriatrician and GNPs, who provide extra information about the patient where needed. Based on the medication review performed by the clinical pharmacist and the discussion with the geriatrician and GNPs, a medication plan is formulated and the proposed changes are recorded in the patient's medical file. Figure 1 provides a flowchart of eligibility for the medication review. 


\section{Patients}

For this study, the medical records of two patient cohorts were compared: one control cohort of patients who did not receive a medication review, and a cohort of patients who did receive a medication review. The inclusion criteria for both cohorts were: 1 . the patients suffered a delirium during hospital stay; 2 . the patients were 70 years or older on the day of admission; and 3. the patients received a consultation from either a geriatrician or GNP. Additionally, patients in the review cohort received a medication review. The control cohort consisted of the medical records of patients admitted to the MUMC+ between 1 January 2014 and 31 December 2014 (before introduction of the medication review), and the review cohort consisted of the medical records of patients admitted to the MUMC+ between 1 July 2015 and 31 December 2016 (after introduction of the medication review). The inclusion of medical records for the review cohort started five months after initiation of the weekly medication review meeting, to avoid start-up problems that could have influenced the study outcomes. To set up comparable group numbers, the length of data collection in both cohorts varies.

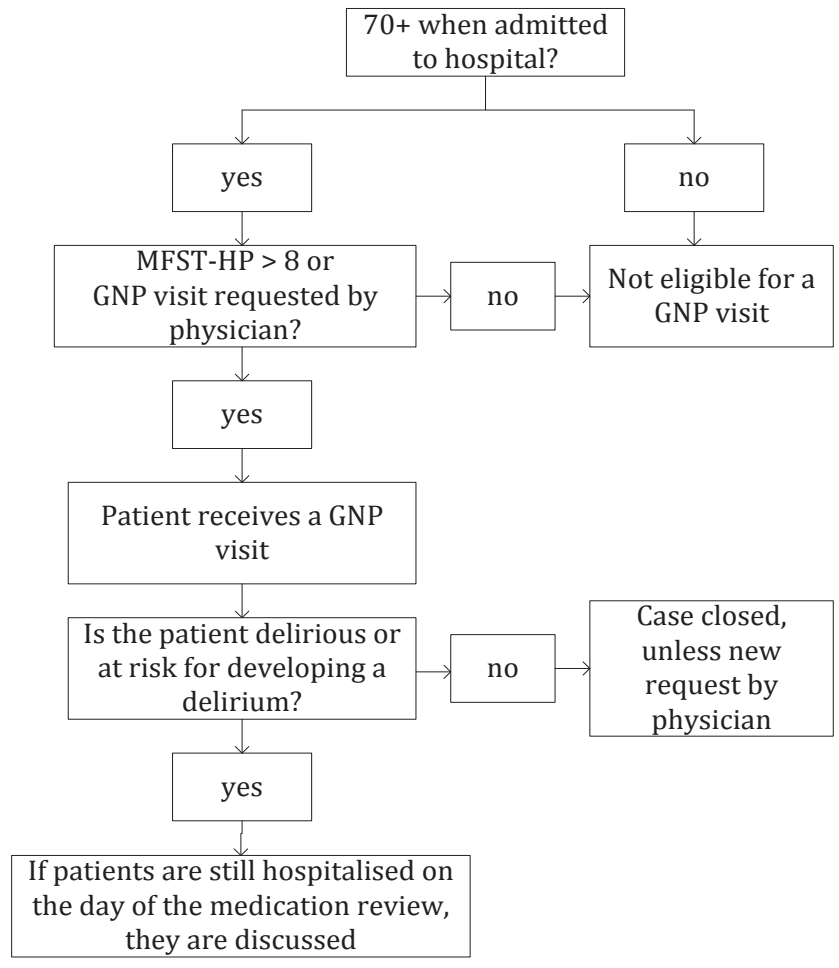

Figure 1. Flowchart of patient eligibility for the medication review. GNP = geriatric nurse practitioner MFST-HP = Maastricht Frailty Screening Tool for Hospitalised Patients 


\section{Data extraction}

\section{Patient data}

The following data/ variables were extracted from the digital medical records by a researcher (author EvV): 1. demographic data: age at hospital admission, sex, living conditions prior to hospital admission (i.e. own home or a nursing home), and reason for admission; 2. baseline data: Charlson Comorbidities Index (CCI), and number and type of medications used at time of the GNP consultation (before the medication review); 3 . the proposed changes in medication, and if and to what extent the proposed changes had been implemented by the patient's treating physician (see section 2.4.2); and 4. adverse outcomes: duration of the delirium, length of hospital stay, in-hospital mortality and discharge destination (back home, to a nursing home, or to a rehabilitation facility).

Duration of the delirium was set as follows: the beginning of the delirium was the date on which the diagnosis of delirium was first mentioned in the digital medical record. The end date of the delirium could be determined by several factors, in decreasing order of importance: 1 . when a physician or GNP noted in the medical record that the delirium was in remission or had passed; 2 . when the Delirium Observation Screening scores (DOS; a tool to screen for delirium and monitor its course and severity ${ }^{20}$ ) remained below three points for three consecutive measurements; 3 . when the patient had died during the delirious episode; or 4 . when the patient had been discharged from the hospital.

\section{Data concerning the medication review}

Authors EP (geriatrician) and LvdV (clinical pharmacist) both independently and blinded to the conclusions of the other assessed all of the proposed medication changes in terms of type of change, and whether or not the proposed change was implemented by the treating physician. After completion, the results of both authors were compared. In case of discrepancy, the patient was re-assessed by authors EP and LvdV together, until consensus was reached. The proposed changes were categorised into "start", "stop", or "change", meaning starting new medication, stopping currently used medication, or changing the dosage, administration mode, or time of administration of a medication currently used by the patient.

Analysis of the implementation of the proposed changes was done on two levels: for each unique proposed change, and per patient, as multiple changes were proposed for most patients. Implementation of the proposed change by the treating physician was categorised either as "yes", "no" or "unknown" (e.g. if the proposed change included medication to be given after discharge, the researchers had no access to this data). On the patient level, the implementation was assessed as "yes" if at least one of the proposed changes for a patient's medication was implemented. If none of the proposed changes were implemented, it was assessed as "no", and if this was unclear, it was assessed as "unknown". The implementation of the 
proposed changes on the patient level was categorised as "yes" if at least one of the proposed changes was implemented, because the authors believe this means the treating physician read and considered the proposed changes, and proceeded to implement what she/he found beneficial for the patient.

\section{Ethics}

The study was approved by the Medical Ethics Committee of Maastricht University / Maastricht University Medical Centre (\#16-4-108).

\section{Statistics}

Baseline characteristics for both cohorts were compared using a two-tailed t-test for continuous data such as age and the $\mathrm{CCI}$, and a chi-square test for nominal data such as sex, living conditions and reasons for admission. The alpha for reason for admission was set to 0.01 to correct for multiple testing. A generalised linear regression model with a negative binomial distribution was used to analyse the differences between the two cohorts for duration of delirium and length of hospital stay as outcome to correct for skewness of the data. A logistic regression was used for in-hospital mortality and discharge destination. All regression models included sex, age, CCI, and number of medications used by the patients. Non-significant covariates were removed from the model stepwise. For the adverse outcomes, an interaction term for cohort and number of medications used was added, as it is expected that especially patients who take a high number of medications may benefit from the medication review. Data were analysed using SPSS version 22 (IBM Corp., Armonk, NY).

\section{Results}

\section{Sample Characteristics}

The inclusion criteria were fulfilled by 125 patients in the control cohort, and 93 patients in the review cohort. Table 1 presents an overview of the reasons for admission of both cohorts. Patients in the review cohort were admitted more often with a hip or femur fracture than patients in the control cohort $(\mathrm{p}=0.01)$. There were no significant differences in other reasons for admission between the two cohorts (alpha for reasons of admission was set to 0.01 to correct for multiple testing). The two cohorts were comparable with respect to age, sex, and CCI. However, patients in the control cohort used on average significantly fewer medications compared to the patients in the review cohort ( 8 vs 10 medications, $\mathrm{p}<0.001$ ) (see Table 2). 
Table 1. Primary reasons for admission ${ }^{a}$ of the patients in both cohorts

\begin{tabular}{lllll}
\hline Reasons for admission & $\begin{array}{l}\text { Total } \\
\mathbf{( N = 2 1 8 )}\end{array}$ & $\begin{array}{l}\text { Control } \\
\text { cohort } \\
\text { (N=125) }\end{array}$ & $\begin{array}{l}\text { Review } \\
\text { cohort } \\
\text { (N=93) }\end{array}$ & p-value \\
\hline Cardiovascular problems & $35(16 \%)$ & $17(14 \%)$ & $18(19 \%)$ & 0.25 \\
Infections & $32(15 \%)$ & $16(13 \%)$ & $16(17 \%)$ & 0.36 \\
Hip or femur fractures & $60(27 \%)$ & $26(21 \%)$ & $34(37 \%)$ & 0.01 \\
General downturn & $15(7 \%)$ & $8(6 \%)$ & $7(8 \%)$ & 0.75 \\
Oncological causes & $8(4 \%)$ & $6(5 \%)$ & $2(2 \%)$ & 0.30 \\
CVA/trauma capitis & $9(4 \%)$ & $8(6 \%)$ & $1(1 \%)$ & 0.05 \\
Delirium or confusion & $17(8 \%)$ & $13(10 \%)$ & $4(4 \%)$ & 0.10 \\
Pulmonary causes (other than infections) & $9(4 \%)$ & $9(7 \%)$ & $0(0 \%)$ & 0.03 \\
Gastro-intestinal and intra-abdominal issues & $17(8 \%)$ & $13(10 \%)$ & $4(4 \%)$ & 0.10 \\
Other & $16(7 \%)$ & $9(7 \%)$ & $7(8 \%)$ & 0.93 \\
\hline
\end{tabular}

aClassification of the reasons for admission into the tabulated categories can be found in Appendix 1 . Differences between the cohorts were calculated using a chi-square test, alpha was set to 0.01 to correct for multiple testing. $\mathrm{CVA}=$ cerebrovascular accident

Table 2. Demographic characteristics and baseline data of the total sample and of the both cohorts (control and review cohorts)

\begin{tabular}{lllll}
\hline & $\begin{array}{l}\text { Total } \\
\mathbf{( N = 2 1 8 )}\end{array}$ & $\begin{array}{l}\text { Control } \\
\text { cohort } \\
(\mathbf{N}=125)\end{array}$ & $\begin{array}{l}\text { Review } \\
\text { cohort } \\
(\mathbf{N}=93)\end{array}$ & p-value \\
\hline Female, n (\%) & $98(45 \%)$ & $58(46 \%)$ & $40(43 \%)$ & $\mathrm{p}=0.62$ \\
Age m, \pm sd (range) & $83 \pm 6(70-98)$ & $82 \pm 6(70-97)$ & $83 \pm 8(70-98)$ & $\mathrm{p}=0.67$ \\
Home-dwellinga, n (\%) & $179(82 \%)$ & $104(83 \%)$ & $75(81 \%)$ & $\mathrm{p}=0.72$ \\
CCI, m \pm sd (range) & $7 \pm 2(3-16)$ & $7 \pm 2(3-16)$ & $7 \pm 2(3-14)$ & $\mathrm{p}=0.82$ \\
Medications ${ }^{\mathrm{b}}, \mathrm{m} \pm$ sd (range) & $9 \pm 4(0-24)$ & $8 \pm 4(1-19)$ & $10 \pm 5(0-24)$ & $\mathrm{p}<0.001$ \\
\hline
\end{tabular}

$\mathrm{CCI}=$ Charlson Comorbidity Index, $\mathrm{m}=$ mean

a Living at home before hospital admission

${ }^{\mathrm{b}}$ Number of medications taken by a patient before the medication review 


\section{Medication review}

All 93 patients in the review cohort received a medication review by the clinical pharmacist and geriatrician. For 75 patients (81\%), changes regarding the medication were proposed, while for 18 patients (19\%) no changes were proposed. Changes were proposed on 180 accounts, with a mean of 1.9 changes per patient (range 0-7). On the level of each unique proposed change, independent of the patient, most changes (62\%) were implemented by the treating physician, $31 \%$ was not implemented, and $7 \%$ is unknown. The proposed changes consisted of starting a new medication in $17 \%$, stopping certain medications in $40 \%$, and changing either the dosage, administration mode, or time of administration in $43 \%$. The majority (40\%), of the proposed changes concerned psychotropic medication, especially stopping or changing (see Table 3 for the details). The proposed changes were implemented for 58 out of the 75 patients $(77 \%)$.

\section{Adverse outcomes}

The duration of delirium in the review cohort was on average 6.91 days shorter compared to the control cohort (8.56 vs 15.47 days respectively, showing a difference of almost 50\%). A significant interaction effect between cohort and number of medications was found for the duration of delirium. Patients in the review cohort taking up to six medications had significantly shorter delirious episodes compared to patients taking up to six medications in the control cohort (mean difference 15.46 days, $\mathrm{p}<0.001$ ). For patients taking between seven and 11 medications the mean difference in duration of delirium between the two cohorts was 3.74 days $(\mathrm{p}=0.07)$. For patients taking 12 or more medications, the mean difference in duration of delirium between the cohorts was 1.81 days $(\mathrm{p}=0.55)$ (see Table 4 for the interaction effect). The other variables in the model (age, sex, CCI) were not significant and thus removed from the model. No significant differences were found for length of stay, in-hospital mortality, or discharge destination. 

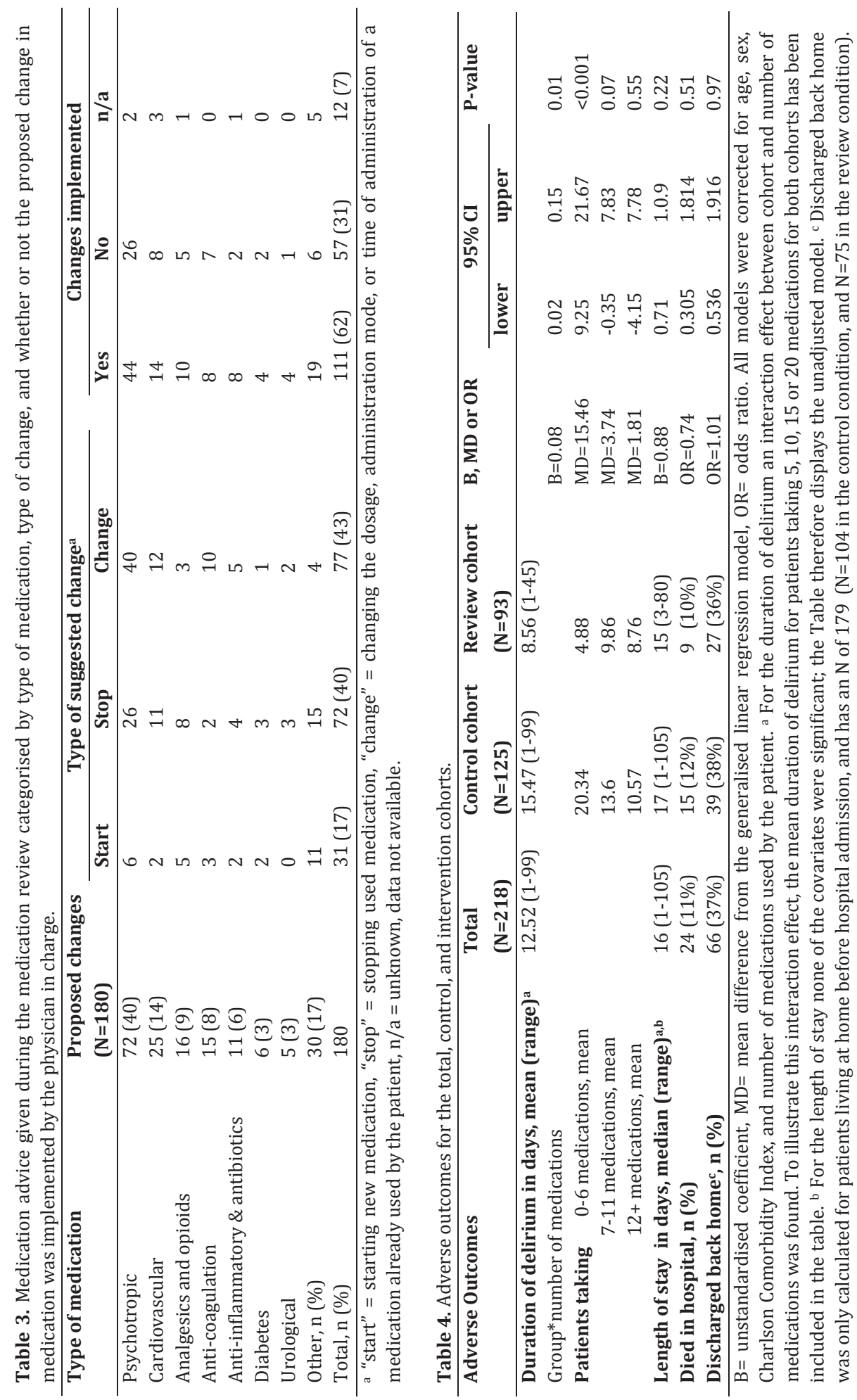


\section{Discussion}

This study examined the benefit of a medication review in addition to standard geriatric care concerning delirium. Several adverse outcomes for patients with delirium (length of stay, duration of delirium, in-hospital mortality and discharge destination) were studied. Furthermore, the medication review and proposed changes to a patient's medication were described in detail: what types of medications were subject to change, and whether or not the proposed changes were implemented by the physicians. An interaction effect of cohort and the amount of medications a patient was taking was found for the duration of delirium: patients taking few medications in the review cohort had significantly shorter delirious episodes than their peers in the control cohort. This effect disappeared when the amount of medications taken by a patient increased to seven or more. No significant differences were found for length of stay, in-hospital mortality or discharge destination.

The initial hypothesis was that the medication review would benefit patients on all the negative outcomes of delirium. It was also hypothesised that this effect would be especially noticeable for patients taking more than six medications, as the chance of drug interactions and negative side effects increases with every additional drug. However, contrary to the hypothesis, the duration of the delirium in the review cohort was reduced significantly for patients taking between zero and six medications. For patients taking between seven and 11 medications, the difference in duration of delirium bordered significance, and for patients taking twelve or more medications there was no significant difference. This indicates that patients with fewer medications might have benefitted more from the medication review. A possible explanation for this could be that the relative impact of a change decreases when the number of medications increases. Another possibility is that patients taking more medications may have more morbidities and may therefore have a delirium which is caused by several factors, which may be more difficult to detect and treat.

The shorter duration of delirium for patients discussed in the review cohort is in line with previous studies that found that geriatric consultation teams focusing on risk factors such as medication and polypharmacy can prevent or shorten a delirium. ${ }^{15}$ Moreover, in addition to being statistically significant, a shorter delirious episode is also highly clinically relevant: a systematic review has shown that longer or persistent delirium is associated with increased delirium severity ${ }^{21}$ and with slower or no functional recovery. ${ }^{22}$ One study found that functional recovery for each day of admission without delirium was inversely correlated with duration of the delirium (i.e. the shorter the duration of the delirium, the better the functional recovery). ${ }^{23}$ Better and swifter functional recovery is not only beneficial for the patient, but could also lead to lower healthcare costs as healthier patients would be more independent, less likely to be admitted to a long-term care facility or readmitted to hospital. 
Despite the shorter duration of delirium among patients taking fewer medications in the intervention cohort, no effects of the medication review were found on the other outcomes (length of stay, mortality and discharge destination). It could be argued that a shorter delirious episode leads to a faster functional recovery and thus a shorter length of hospital stay. However, we did not find any significant differences in length of hospital stay between the two cohorts in the current study. With regards to mortality, we only included data about in-hospital mortality and did not examine mortality post-discharge. Delirium can lead to increased risk of death within 12 months after the delirium. ${ }^{5}$ It could be assumed that patients with a shorter delirious episode had lower mortality rates post discharge, but those conclusions are beyond the scope of the present study. Discharge destination was also similar in both cohorts. One may assume that patients who took fewer medications (1-6) were the cohort's healthier patients, of whom most were able to return to their previous living arrangements after hospital discharge.

In addition to the clinical effects, the costs of delirium have been estimated to be $\$ 164$ billion in the USA on a yearly basis. ${ }^{8}$ Caring for delirious patients can be burdening for nurses ${ }^{24}$ and may be challenging. ${ }^{25} \mathrm{~A}$ German study showed that nurses spend an average of 240 minutes extra and physicians 66 minutes extra on the care of delirious patients compared to non-delirious controls, and the costs were estimated to be about $€ 1200$ extra per day for each patient with hyperactive delirium. ${ }^{9}$ Taking this information into account, and considering that between $29 \%$ and $64 \%$ of older hospitalised patients suffer a delirium during their hospital stay, ${ }^{2}$ reducing the duration of a patient's delirium could decrease health care costs. Although a cost-effectiveness analysis was beyond the scope of this study, it is not improbable that this might be a cost-effective intervention, considering the shorter delirious episodes.

The majority of the changes (40\%) in medication were made for psychotropic medication, followed by cardiovascular medication (14\%). Most of the suggested changes for all types of medication were either to stop the medication or to change it; only a few changes (17\%) involved starting a new medication. This suggests that many patients take unnecessary medication, especially psychotropic medication, such as benzodiazepines, which can be highly addictive, lose effectiveness after prolonged use, and may increase the risk of falling in older people due to their sedative effects. About two thirds of all proposed changes were implemented by the treating physicians. At least one or more of the proposed changes were implemented for about $70 \%$ of the patients. This indicates that in some cases physicians may have deliberately made the choice to implement some of the proposed changes and disregard others, possibly for clinical or treatment reasons. Another reason for not implementing a proposed change may have been that the treating physician did not read the recommendations made by the review team. However, we could not uncover what the reasons were for not implementing the proposed changes. 
Medication reviews are part of the national and international guidelines for the prevention and management of delirium in hospital patients. However, these guidelines are not common practice for all older patients: in our study, medication review was only done for those patients who were identified as frail using a frailty index, or patients for whom a physician requested a review. This means that many patients, including delirious patients, did (and do) not receive a medication review, despite the guidelines. The results from this study show that a medication review for all patients can be beneficial with regard to the duration of a delirium. These results could be used as evidence of best practice and might help to show the added value of a hospital-wide medication review, thus creating more awareness of the importance of a medication review for all older patients. This awareness could eventually also lead to a change in prescribing practice throughout the hospital, where physicians, policy makers, other health care workers, and patients and family members become more aware of possible dangers of medications and their interaction with other medications.

\section{Limitations and Strengths}

Some issues regarding the methodology of the present study need to be considered. A retrospective cohort study was performed, instead of a randomised controlled trial (RCT). Although this choice was mainly made for practical reasons -the medication review had already been introduced at the start of the study-it could also be argued that an RCT in this case may not have been ethical, as this would mean withholding patients from care that may benefit them without causing any negative side-effects. A downside to the retrospective design of the current study was that delirium diagnosis could not be standardised using a diagnostic instrument. Instead, delirium was diagnosed by two GNPs based on the Diagnostic and statistical manual of mental disorders $4^{\text {th }}$ edition (DSM-IV) ${ }^{1}$ criteria for delirium. Although the GNPs are trained to diagnose delirium and patients are discussed with a geriatrician, the quality of the study could have been improved if delirium diagnosis was standardised beforehand. Moreover, causality cannot be implied when using a retrospective design. Furthermore, the cohorts were different in size: the control cohort was larger than the medication review cohort (125 vs 93 patients, respectively). This was because not all patients who had suffered a delirium during their hospitalisation could be included in the medication review cohort. Medication reviews were only conducted on Thursdays, thus patients who were discharged back home before the medication review could take place were not included in the review cohort. Moreover, due to the retrospective nature of this study, power calculations were not performed. It would be advisable, therefore, to replicate the study in an RCT and with cohort sizes based on power calculations.

Strengths of the study are several. First, the retrospective nature of this study let us examine an intervention in daily practice, outside of a controlled research setting. Our results are therefore not influenced by a potential bias of the physicians to 
implement the suggested changes to the medication. It also proves that such medical interventions are viable and can be effective outside a research setting. Second, there was a relatively short period (five months) between the end of the control cohort and the start of the review cohort. This minimises the chance that a part of the effects could be attributed to external factors, as there were no changes in national guidelines or standard procedures within the hospital during that period. Third, relevant medical records in both cohorts were identified in the same manner and had the same inclusion criteria, minimising the chances of selection bias.

\section{Conclusion}

Our findings indicate that a medication review, carried out by a clinical pharmacist and a geriatrician, may significantly decrease the duration of the delirium during hospitalisation, especially for patients taking few medications. A clinical pharmacist, therefore, is a valuable addition to a geriatric team, as they have a deep understanding of how medications work and interact, and how to best review a patient's medication list. Based on the clinical benefits of a shorter delirious episode, we recommend that a team consisting of at least a medical pharmacist and a geriatrician perform medication reviews for all older patients in a hospital. This could possibly also contribute to the prevention of delirium. 


\section{References}

1. American Psychiatric Association: Diagnostic and Statistical Manual of Mental Disorders (4th ed.), 4. Washington, DC, American Psychiatric Association, 2000

2. Inouye SK, Westendorp RGJ,Saczynski JS: Delirium in elderly people. Lancet 2014; 383:911-922

3. Davis DH, Muniz Terrera G, Keage H, et al: Delirium is a strong risk factor for dementia in the oldest-old: a population-based cohort study. Brain 2012; 135:2809-2816

4. Krogseth M, Watne LO, Juliebo V, et al: Delirium is a risk factor for further cognitive decline in cognitively impaired hip fracture patients. Arch Gerontol Geriatr 2016; 64:38-44

5. Witlox J, Eurelings LS, de Jonghe JF, et al: Delirium in elderly patients and the risk of postdischarge mortality, institutionalization, and dementia: a metaanalysis. JAMA 2010; 304:443-451

6. Bail K, Goss J, Draper B, et al: The cost of hospital-acquired complications for older people with and without dementia; a retrospective cohort study. BMC Health Serv Res 2015; 15:91

7. Leslie DL,Inouye SK: The importance of delirium: economic and societal costs. J Am Geriatr Soc 2011; 59:S241-S243

8. Leslie DL, Marcantonio ER, Zhang Y, et al: One-year health care costs associated with delirium in the elderly population. Arch Intern Med 2008; 168:27-32

9. Weinrebe W, Johannsdottir E, Karaman M, et al: What does delirium cost? : An economic evaluation of hyperactive delirium. Z Gerontol Geriatr 2015;

10. Inouye SK, Bogardus ST, Charpentier PA, et al: A multicomponent intervention to prevent delirium in hospitalized older patients. N Engl J Med 1999; 340:669-676

11. Marcantonio ER, Flacker JM, Wright RJ, et al: Reducing delirium after hip fracture: a randomized trial. J Am Geriatr Soc 2001; 49:516-522

12. Bjorkelund $\mathrm{KB}$, Hommel A, Thorngren $\mathrm{KG}$, et al: Reducing delirium in elderly patients with hip fracture: a multi-factorial intervention study. Acta Anaesthesiol Scand 2010; 54:678-688

13. Inouye S, Robinson T,Blaum C: Postoperative delirium in older adults: best practice statement from the American Geriatrics Society. J Am Coll Surg 2015; 220:136-148 e131

14. Milisen K, Foreman MD, Abraham IL, et al: A nurse-led interdisciplinary intervention program for delirium in elderly hip-fracture patients. J Am Geriatr Soc 2001; 49:523-532

15. Rizk P, Morris W, Oladeji $\mathrm{P}$, et al: Review of postoperative delirium in geriatric patients undergoing hip surgery. Geriatr Orthop Surg Rehabil 2016; 7:100-105

16. Vidan M, Serra JA, Moreno C, et al: Efficacy of a comprehensive geriatric intervention in older patients hospitalized for hip fracture: a randomized, controlled trial. J Am Geriatr Soc 2005; 53:1476-1482

17. Ahmed S, Leurent B,Sampson EL: Risk factors for incident delirium among older people in acute hospital medical units: a systematic review and metaanalysis. Age Ageing 2014; 0:1-8 
18. Warnier R, van Rossum E, van Leendert J, et al: Screening for frailty in hospitalized older adults: reliability and feasibility of the Maastricht Frailty Screening Tool for Hospitalized Patients (MFST-HP). Res Gerontol Nurs 2016; 9:243-251

19. Gallagher P, Ryan C, Byrne S, et al: STOPP (Screening Tool of Older Person's Prescriptions) and START (Screening Tool to Alert doctors to Right Treatment). Consensus validation. Int J Clin Pharmacol Ther 2008; 46:72-83

20. Schuurmans MJ, Shortridge-Baggett LM,Duursma SA: The Delirium Observation Screening Scale: a screening instrument for delirium. Res Theory Nurs Pract 2003; 17:31-50

21. Dasgupta M,Hillier LM: Factors associated with prolonged delirium: a systematic review. Int Psychogeriatr 2010; 22:373-394

22. Kiely DK, Jones RN, Bergmann MA, et al: Association between delirium resolution and functional recovery among newly admitted postacute facility patients. J Gerontol A Biol Sci Med Sci 2006; 61:204-208

23. Speciale $S$, Bellelli G, Lucchi E, et al: Delirium and functional recovery in elderly patients. J Gerontol A Biol Sci Med Sci 2007; 62:107-108; author reply 108

24. Mc Donnell S, Timmins F: A quantitative exploration of the subjective burden experienced by nurses when caring for patients with delirium. J Clin Nurs 2012; 21:2488-2498

25. Day J, Higgins I, Keatinge D: Orientation strategies during delirium: are they helpful? J Clin Nurs 2011; 20:3285-3294 


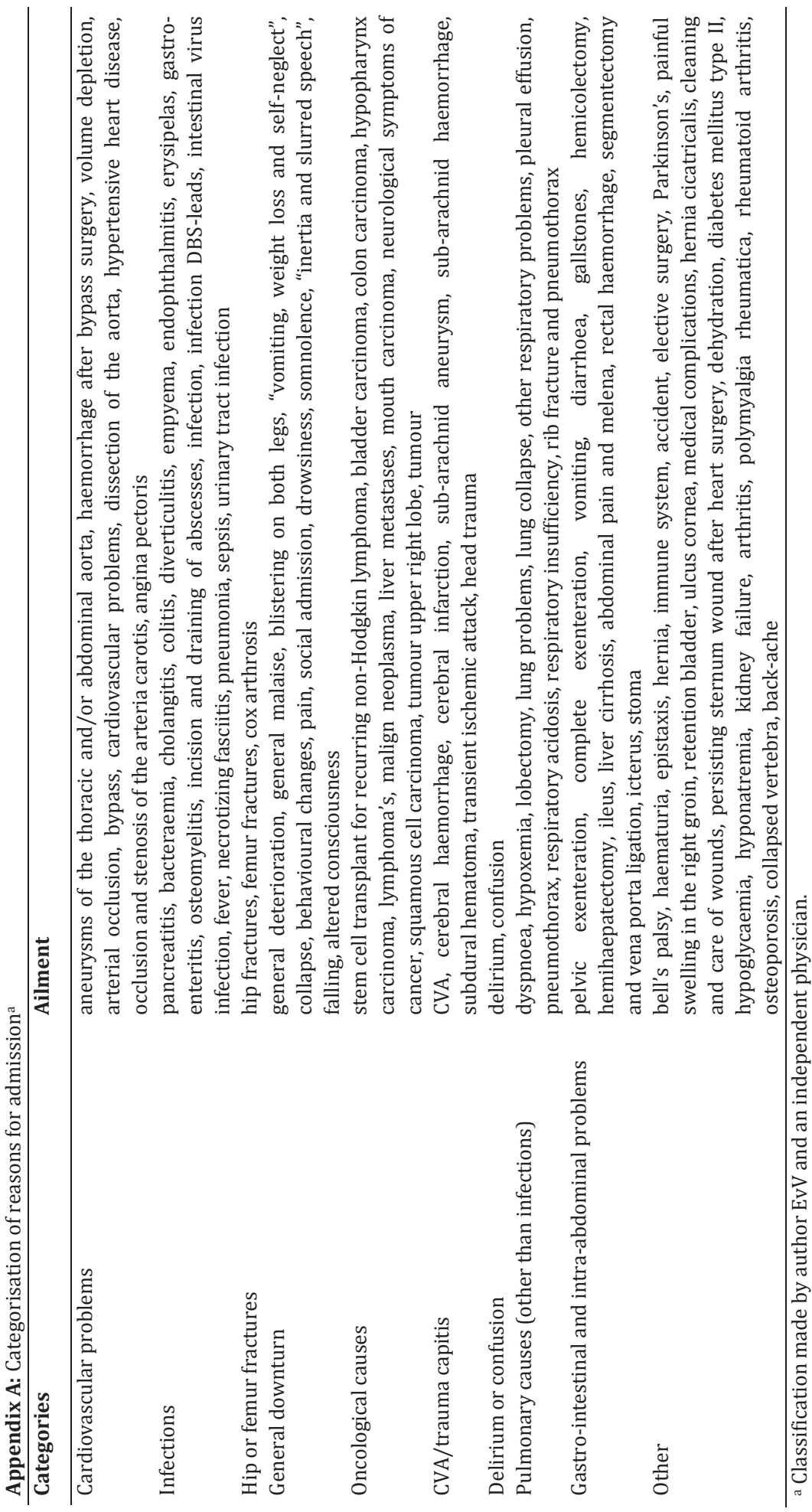


One day we shall die.

But on all the other days we shall be alive

- P. O. Enquist (The Royal Physician's Visit, 1999)

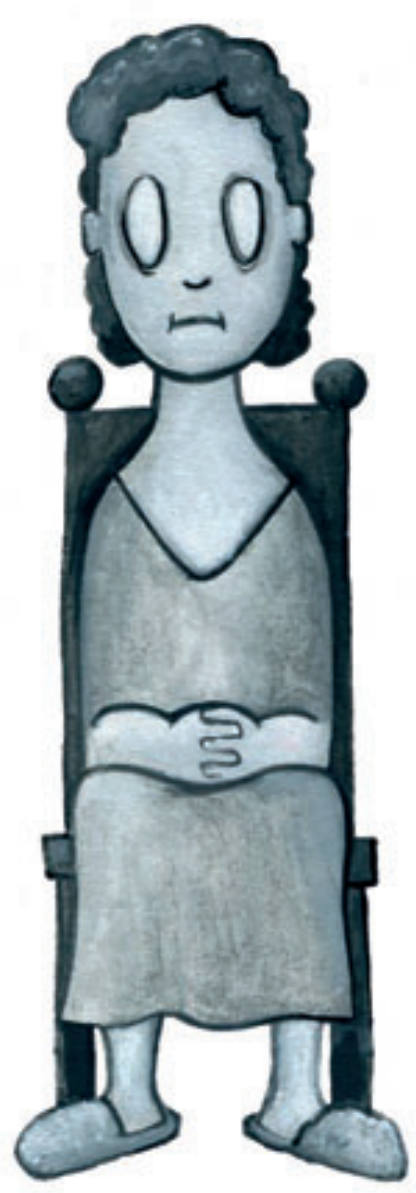




\section{CHAPTER 5 \\ Can education improve clinical practice concerning delirium in older hospitalised patients? Results of a pre- test post-test study on an educational intervention for nursing staff}

This chapter has been published as:

Eveline L van Velthuijsen, Sandra MG Zwakhalen, Ron Warnier, Ton Ambergen, Wubbo J Mulder, Frans RJ Verhey and Gertrudis IJM Kempen. Can education improve clinical practice concerning delirium in older hospitalised patients? Results of a pre-test post-test study on an educational intervention for nursing staff. BMC Medical Education. 2018; 18(1):59 


\begin{abstract}
Background: Delirium is a common and serious complication of hospitalisation in older adults. It can lead to prolonged hospital stay, institutionalisation, and even death. However, it often remains unrecognised or is not managed adequately. The aim of this study was to evaluate the effects of an educational intervention for nursing staff on three aspects of clinical practice concerning delirium in older hospitalised patients: the frequency and quality of screening for delirium using the 13-item Delirium Observation Screening score (DOS), and the frequency of geriatric consultations requested for older patients. The a priori expectations were that there would be an increase in all three of these outcomes.

Methods: We designed an educational intervention and implemented this on two inpatient hospital units. Before providing the educational session, the nursing staff was asked to fill out two questionnaires about delirium in older hospitalised patients. The educational session was then tailored to each unit based on the results of these questionnaires. Additionally, posters and flyers with information on the screening and management of delirium were provided and participants were shown where to find additional information. Relevant data (outcomes, demographics and background patient data) were collected retrospectively from digital medical files. Data was retrospectively collected for four different time points: three pre-test and one post-test.

Results: There was a significant increase in frequency of delirium screening $(\mathrm{P}=0.001)$, and both units showed an increase in the quality of the screening. No significant effect of the educational intervention was found for the proportion of patients who received a geriatric consultation $(\mathrm{P}=0.083)$.

Conclusion: The educational intervention was successful in making positive changes in clinical practice: after the educational session an improvement in the frequency and quality of screening for delirium was observed. A trend towards an increase in the proportion of geriatric consultations for older hospitalised patients was also observed.
\end{abstract}




\section{Background}

Delirium was first described by Hippocrates about 2.500 years ago. However, despite its long history, the disorder still remains greatly unrecognised in older hospitalised patients, and management is often not done in accordance to the national or international guidelines.1,2 This failure to recognise or adequately manage a delirium is partially attributable to a lack of the necessary knowledge and skills among the medical and nursing staff. ${ }^{3-5}$ Indeed, medical and nursing schools often do not put sufficient emphasis on the education of delirium in their curriculum. ${ }^{6}$

Delirium is a serious and common disorder, affecting up to $29-64 \%$ of older (65+) hospitalised patients. It is an acute brain failure characterised by an acute change in consciousness and a decline in cognitive functions with reduced ability to focus, sustain or shift attention. The disorder is also often accompanied by perceptual disturbances, such as audio-visual hallucinations and delusions, and motor symptoms, such as agitation or slowing of movement and apathy. ${ }^{7}$ It is associated with increased mortality, longer hospital stay, slower functional recovery, more readmissions, and increased chances of developing dementia. ${ }^{1}$ Timely recognition and adequate management should therefore be an important part of hospital care, especially for older hospital patients at risk of developing a delirium. The nursing staff is especially important in this process of recognition and management: they spend more time with patients than physicians do, and are often more likely to see sudden changes in behaviour or cognition. Educational interventions could possibly have, therefore, the best results when aimed at the nursing staff.

Educational interventions aimed at increasing delirium knowledge in nursing staff have been previously developed and studied 8 , including e-learning, ${ }^{9,10}$ where the main outcomes were mostly related to an increase in knowledge measured with a questionnaire. ${ }^{8-10}$ However, if one wants to bring about a change in clinical practice, it may be not enough to just increase knowledge on a certain topic. ${ }^{8}$ According to the PRECEDE model (Predisposing, Reinforcing and Enabling Constructs in Educational Diagnosis and Evaluation) developed by Green and colleagues, an educational intervention may only become effective (i.e. bring about a change in clinical practice) when it should not only provide information, but also should reinforce and enable participants to use the acquired knowledge (i.e. by using reminders and feedback, providing flowcharts, guidelines etc.). ${ }^{11}$

The goal for the present study was to evaluate the effects of an educational intervention (based on the PRECEDE model) for nursing staff on the proportion of patients screened for delirium, the appropriate use of this screening and the proportion of patients being referred for a geriatric consultation. The a priori expectations were that there will be an increase in proportion of patients screened for delirium, an increase in the quality of the screening, and an increase in geriatric consultations. 


\section{Methods}

\section{Design, setting and participants}

A pre-test post-test study was carried out at two inpatient medical units of the Maastricht University Medical Centre+ (MUMC+), a 715 bed university teaching hospital in the south of the Netherlands.

The nursing staff from two inpatient units participated in the educational intervention: a combined unit (including dermatology, plastic surgery, nuclear medicine and urology, henceforth referred to as unit A) and a cardiology unit (henceforth referred to as unit B). Twenty six participants worked at unit A, and 33 participants at unit B. No inclusion criteria were set for the nursing staff to participate.

\section{Educational intervention}

The educational intervention consisted of five different steps, in which the three components of the PRECEDE model (predisposing, reinforcing, and enabling) are incorporated. Figure 1 provides an overview of the educational intervention, including the different components of the PRECEDE model and a timeline of the individual steps. In step 1, the nursing staff of the two units were invited to complete two questionnaires: the Delirium Knowledge Questionnaire (DKQ) and the Strain in Care for Delirium Index (SCDI). The DKQ was designed by Detroyer and colleagues ${ }^{10}$ and was used to assess existing levels of knowledge on delirium of the nursing staff. The SCDI was developed by Milisen and colleagues, ${ }^{12}$ and was used to assess the perceived burden for caring for delirious patients. In step 2, the educational session was given. The educational session was tailored to each unit according to the knowledge gaps made apparent by the questionnaires from step 1. The session included information on risk factors, precipitating factors, preventive measures, and delirium management. It was presented using slides, videos of patients with a hyperactive or hypoactive delirium, examples from clinical practice, and an interactive discussion. In step 3, which occurred at the end of the educational session, the Geriatric Nurse Practitioner (GNP), specialised in delirium, providing the educational session (author RMJW) showed the participants how and where to find the guidelines and more information about delirium on the hospital's intranet. The GNP also gave his contact number so the nursing staff could always contact him concerning questions or doubts related to delirium in older patients. In step 4, directly after the educational session, posters about screening and management of delirium were placed in the nurses' office. Additionally, informational flyers for informal caregivers were placed on the units. In step 5, two weeks after attending the educational session, the nursing staff was asked to fill out the DKQ and SCDI again. 


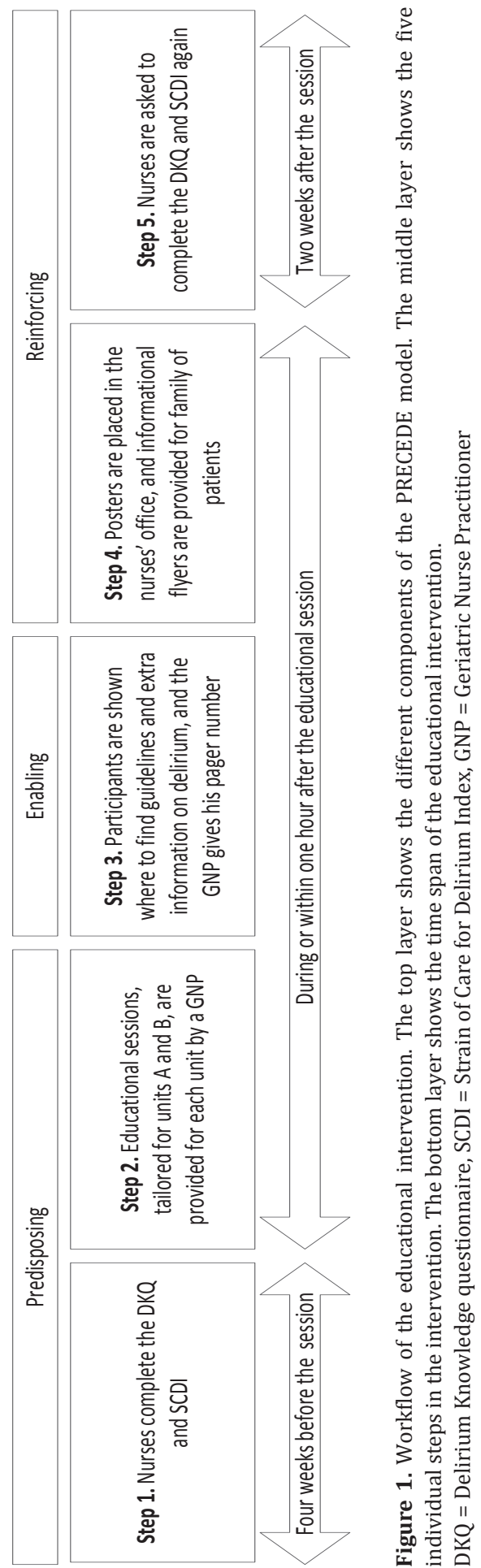




\section{Providing the educational sessions}

Before planning the educational sessions, authors ELvV and RMJW consulted the individual unit leaders about when and where the educational session could best be held to maximise the reach of the educational session. For unit A, the unit leader decided it was best to incorporate the educational session in a monthly mandatory unit meeting. For unit B, it was decided to hold the session twice on two different dates, and to make participation voluntary. Thus, one session was held for unit A and two sessions were held for unit B. All participants attended the session only once. The educational sessions were delivered in March and April 2017.

\section{Data collection}

\section{Procedure}

All data was collected by author ELvV. The data collected from patient files (i.e. three outcomes and patient demographics) was collected retrospectively in June 2017.

Data was collected for four different time points: three pre-test (February 2016, May 2016 and February 2017) and one post-test (May 2017). We included three pre-test measurements spread across a longer time period to be able to identify any pre-existing trends or seasonal differences in the outcome measures. Patient files were included for data collection if the patients were 70 years or older at time of hospital admission and were admitted to either unit A or unit B.

\section{Main outcomes}

The main outcomes of the study were: 1 . the proportion of older patients on the unit screened for delirium using the 13-item Delirium Observation Screening score (DOS); 2. the proportion of DOS scores completed correctly; and 3. the proportion of older patients receiving a geriatric consultation.

All DOS scores and the date and time at which the DOS was filled out are all recorded in the patients' medical file. The DOS score is initiated either by the nursing staff, a physician or by a GNP after a geriatric consultation. The criteria for keeping DOS scores is when patients are delirious or at risk of developing a delirium. A DOS score was considered correct if it was completed three times a day for at least three consecutive days. ${ }^{13}$ The third outcome was the proportion of patients receiving a geriatric consultation. Nursing staff can request a geriatric consultation with a GNP whenever they feel this is necessary. During a geriatric consultation, a GNP visits the patient for an assessment of frailty and risk of delirium. After the consultation, advice on all factors concerning geriatric care (hydration, nutrition, physical therapy, fall prevention etc.) is given to the nursing staff. In addition, a geriatrician and a clinical pharmacist provide a medication 
review for the patient. This geriatric consultation is also recorded in the patients' digital medial file.

\section{Demographic and background data and feedback}

Demographic and background data from the nursing staff (gender, age, years of experience, and any previous delirium education) were obtained through a list of questions attached to the questionnaires. Demographic and background data for the patients (age, sex, length of stay (LoS, in days)), proportion of patients with a delirium, and duration of the delirium (DoD, in days)) were obtained through the patients' digital medical files. LoS was counted as the number of days between the day of admission and the day of hospital discharge. DoD was counted from the date on which the diagnosis of delirium was first mentioned in the digital patient file, till, in order of importance: 1. a physician or a GNP noted in the patient files that the delirium was in remission or had passed; 2. pharmacological treatment for the delirium was ceased because of abating symptoms; 3. the DOS remained below three points for three consecutive measurements; 4. the patient had died during hospital admission; or 5 . the patient had been discharged from the hospital.

Lastly, we collected feedback from the nursing staff about the educational intervention during the second round of questionnaires on an extra page.

\section{Ethics and Consent}

The study was assessed and approved by the Medical Ethical Committee of the MUMC+ (\#16-4-212). No consent was deemed necessary for the nursing staff, as the delirium training was considered as additional internal education the MUMC+ offers to its staff. No written consent for the patient data was needed, as in the Netherlands all university teaching hospitals have an opt-out system for the use of anonymous patient data for scientific research (i.e. patients automatically consent to the use of their anonymous data for research upon admission, unless they explicitly object).

\section{Analyses}

Statistical analyses were carried out for two main outcome measures: proportion of patients screened using the DOS, and proportion of patients who received a geriatric consultation. No statistical tests were carried out for the proportion of patient with correct DOS scores due to the very low number of correct DOS scores pre-test (0-1). Demographic and background data of the patients were also not subjected to statistical testing, as these data were not expected to influence the main outcomes.

First data from the three pre-test time points (February 2016, May 2016 and February 2017) were compared to each other to check for any significant differences between these three time points to establish a baseline. If the pre-test 
data did not differ significantly between each other, we assumed that any significant changes found between the pre- and post-test situation could be attributed to the educational intervention. In this case, the data from all three pretest moments were combined to form one pre-test group. In case significant differences would be found between the three pre-test time points, a stable baseline could not be assumed, and any differences between the pre-test and posttest conditions could not be automatically attributed to the intervention. The effect of the educational intervention on the proportion of patients screened using the DOS and proportion of patients who received a geriatric consultation were analysed using logistic regression. The basic model included time point (i.e. pre-test vs post-test), the hospital unit (i.e. A and B) and an interaction between time point and unit. If any of the variables or the interaction proved to be non-significant, the analysis was done again with only the significant variables in the model. Alpha was set at 0.05. Data was analysed using SPSS (IBM corp.) version 24.

\section{Results}

\section{Nursing staff}

A total of 34 nursing staff members participated in the- educational intervention: $16(62 \%)$ staff members from unit A and 18 (55\%) from unit B. Most of the participants were female $(\mathrm{N}=31)$. The mean age was 34 (range 20-60), with an average work experience of 11 years (range 1-39). Five nursing staff members had had some delirium education in the last five years.

\section{Patients}

The files of 544 patients were included: 385 in the pre-test condition and 159 in the post-test condition. The three pre-test time points did not differ significantly from each other, thus the three pre-test time points were grouped together to form one pre-test group. See Table 1 for all demographic and background patient data.

Table 1. Demographic and background data of the patients

\begin{tabular}{|c|c|c|c|c|}
\hline \multirow{2}{*}{$\begin{array}{l}\text { Time } \\
\text { Unit }\end{array}$} & \multicolumn{2}{|c|}{ Pre-test $(\mathrm{N}=385)$} & \multicolumn{2}{|c|}{ Post-test $(\mathrm{N}=159)$} \\
\hline & $A(n=164)$ & $B(n=221)$ & $A(n=69)$ & $B(n=90)$ \\
\hline Sex, \%f & 39.0 & 44.3 & 26.1 & 45.6 \\
\hline $\begin{array}{l}\text { Age, } m \pm s d \\
\text { (range) }\end{array}$ & $\begin{array}{l}78.2 \pm 5.9 \\
(70-95)\end{array}$ & $\begin{array}{l}79.5 \pm 6.4 \\
(70-99)\end{array}$ & $\begin{array}{l}79.6 \pm 5.6 \\
(70-91)\end{array}$ & $\begin{array}{l}80.3 \pm 7.3 \\
(70-101)\end{array}$ \\
\hline $\begin{array}{l}\text { LoS, } m \pm s d \\
\text { (range) }\end{array}$ & $\begin{array}{l}6.9 \pm 7.8 \\
(1-64)\end{array}$ & $\begin{array}{l}9.3 \pm 10.2 \\
(1-90)\end{array}$ & $\begin{array}{l}7 \pm 6.6 \\
(1-37)\end{array}$ & $\begin{array}{l}14.1 \pm 12.8 \\
(1-60)\end{array}$ \\
\hline Delirium, n(\%) & $19(11.6)$ & $31(14.0)$ & $8(11.6)$ & $13(14.4)$ \\
\hline $\begin{array}{l}\text { DoD, n; m } \pm s d \\
\text { (range) }\end{array}$ & $\begin{array}{l}4.79 \pm 2.3 \\
(1-9)\end{array}$ & $\begin{array}{l}8.5 \pm 12.1 \\
(1-68)\end{array}$ & $\begin{array}{l}3.9 \pm 2.9 \\
(1-9)\end{array}$ & $\begin{array}{l}16 \pm 14.4 \\
(1-49)\end{array}$ \\
\hline
\end{tabular}

LoS = Length of hospital stay, DoD = Duration of delirium (in days), sd = standard deviation, $\mathrm{m}=$ mean. 


\section{Main outcomes}

The three outcomes were: 1 . the proportion of older patients being screened using the DOS; 2. the number of DOS assessments being performed correctly; and 3. the proportion of patients being referred for a geriatric consultation. A logistic regression was carried out to ascertain the effects of the educational intervention on the proportion of patients who were screened for delirium using the DOS. The variables "unit" and the interaction "unit x intervention" were deleted from the model after the first analysis because neither had a significant effect on the outcomes. Also, the main outcomes did not differ significantly between the timepoints; therefore, the pre-test data was combined to form one pre-test group. Thus, the final regression model comprised only the intervention as independent variable.

The proportion of patients who were screened for delirium increased significantly in both units: from $7.9 \%$ to $14.5 \%$ in unit A and from $9.0 \%$ to $22.2 \%$ in unit B: for both units combined the odd's ratio post-test versus pre-test $=2.481(95 \%$ confidence interval $=1.454-4.231) P=.001$ (see Table 2 and Figure 2.1), indicating patients were about 2.5 times more likely to be screened for delirium after the educational intervention compared to before the educational intervention. Moreover, an increase in the number of patients for whom the DOS was performed correctly (i.e. three times a day for at least three consecutive days) on both units is noticed after the educational sessions, as shown in Table 2 and Figure 2.2.

A similar logistic regression model was used to analyse the effect of the intervention on the proportion of patients who received a geriatric consultation. This final model solely contained the variable of time (i.e. pre-test vs post-test). There was a slight though non-significant trend toward an increase in geriatric consultations after the educational intervention: odd's ratio $=1.623$ (95\% confidence interval $=0.938-2.809$ ) $P=.083$ (see Table 2 and Figure 2.3).

Table 2. Changes in clinical practice

\begin{tabular}{|c|c|c|c|c|}
\hline \multirow{2}{*}{$\begin{array}{l}\text { Time } \\
\text { Unit }\end{array}$} & \multicolumn{2}{|c|}{ Pre-test $(\mathrm{N}=385)$} & \multicolumn{2}{|c|}{ Post-test $(\mathrm{N}=137)$} \\
\hline & $A(n=164)$ & $B(n=221)$ & $A(n=69)$ & $B(n=90)$ \\
\hline Using DOSa, n (\%) & $13(7.9)$ & $20(9.0)$ & $10(14.5)$ & $20(22.2)$ \\
\hline DOS correctly used ${ }^{b}, \mathbf{n}$ & 1 & 1 & 5 & 6 \\
\hline Geriatric consultation, n (\%) & $15(9.1)$ & $23(10.4)$ & $7(10.1)$ & $17(18.9)$ \\
\hline
\end{tabular}




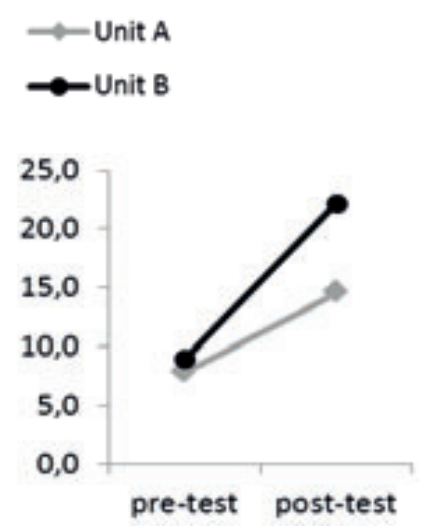

Figure 2.1. Proportion of older patients screened using the Delirium Observation Screening (DOS), separated per unit (A and B).

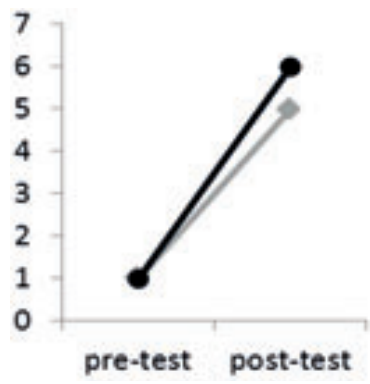

Figure 2.2. Number of DOS scores performed correctly (three times a day for at least three consecutive days) separated per unit (A and B).

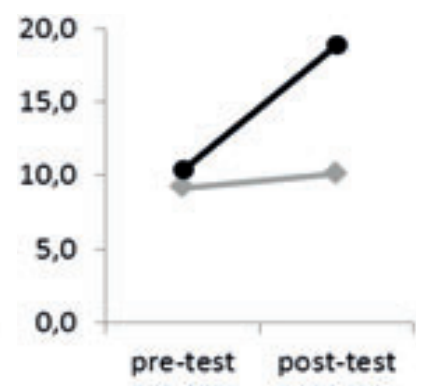

Figure 2.3. Proportion of older patients who received a geriatric consultation per unit (A and B).

\section{Feedback from the participants}

Overall, the educational intervention was considered to be useful and insightful by the nursing staff; especially the use of videos, examples from clinical practice, and the lively discussion. Some participants missed practical guidelines on how to handle aggressive or very hyperactive patients.

\section{Discussion}

The aim of this study was to assess the impact of an educational intervention for nursing staff on the clinical practice regarding screening and management of delirium in older hospitalised patients. We found a statistically significant increase in the proportion of patients screened for delirium using the Delirium Observation Screening score (DOS) in both studied units; patients were almost 2.5 times more likely to be screened for delirium with the DOS after the educational intervention had been delivered. Additionally, the number of patients for whom the DOS was performed correctly (i.e. three times a day for at least three consecutive days) increased notably in both units (from one correct DOS pre-test, to five or six posttest). The proportion of patients who received a geriatric consultation did not increase significantly after the educational intervention, though a trend towards more geriatric consultations was observed.

It is difficult to compare our outcomes to previous studies, as very few studies on the education of nursing staff also examined changes in clinical practice. To our knowledge, just one study, a delirium e-learning programme for nurses conducted 
by Van de Steeg and colleagues, examined the proportion of older patients screened for delirium using the DOS. ${ }^{14}$ This study included participants from 18 hospitals in the Netherlands, and also found a significant increase in the usage of the DOS and screening for frailty in older patients, which is consistent with our findings. Most other studies of educational interventions that have examined the implications for clinical practice were mostly interprofessional educational interventions for physicians and nursing staff,, ${ }^{15-17}$ or were part of a multicomponent intervention, 18,19 and were not aimed only at the nursing staff. These studies showed that education combined with some reform of the regular care system (e.g. the introduction of a geriatric nurse or a care pathway) may increase delirium detection and improvement of delirium management. However, interprofessional education for physicians and nursing staff, including reorganisations or changes in care guidelines may be difficult to implement throughout a hospital.

Although the proportion and quality of the screenings increased significantly after the educational intervention, we did not find a similarly positive result for the proportion of patients receiving a geriatric consultation. An important part of screening is the correct interpretation and evaluation of the DOS scores and consequently a correct course of action. In case of a delirium or suspected delirium, a geriatric consultation should be requested. As can be observed from Figure 2.2, the proportion of geriatric consultations increased more in unit B compared to Unit A. It might be the case that the sense of urgency for delirium was slightly less present for the nursing staff in unit A compared to unit B, or that the nursing staff in unit A was more reluctant to request a geriatric consultation for their patients, which may have led to the trend in increased geriatric consultations instead of a significant increase. Alternatively, it could be that although more patients were screened, this did not result in an increase of delirium diagnoses and consequently no increase in geriatric consultations.

Looking at the results, we noticed that the improvements in unit $B$ were a bit higher than in unit A, though the results did not differ significantly from each other. These small differences could possibly be attributed to two things: the difference in patient population - unit A is a combination of many specialties, while unit B is a cardiology unit- and a difference in attitude or "culture" among the nursing staff in the two units. Anecdotally, during the educational sessions we perceived a greater willingness to cooperate from the staff on unit B, compared to the staff on unit A: more questions were asked, more patient situations and experiences were shared, and more feedback was provided during the educational sessions on unit B compared to unit A. Additionally, the leader of unit B did not make the delirium education mandatory, but the nursing staff was free to participate, while for unit A, the unit leader planned the delirium education as part of a mandatory staff meeting. The willingness to cooperate and the openness for change could be considered very important aspects and possibly overlooked in educational interventions, including the present one. A change in behaviour is likely more prone to crystallise in an environment where this is encouraged and facilitated or driven by intrinsic 
motivation (staff members are free to attend the sessions), compared to externally enforced behaviour (a mandatory staff meeting). As such, it is important to take into account the attitude of the people for whom the education was designed, and, where possible, make sure the unit leaders understand the importance and added value of an educational intervention and openly support and encourage participation. Another option could be to appoint delirium "champions" or "key nurses", who receive more rigorous delirium training, and can provide support to the rest of the nursing staff when dealing with delirious patients. Previous studies incorporating such champions have been found to bring about change in delirium detection and management, ${ }^{20}$ or can play a pivotal role in adapting new ways to work with older patients. ${ }^{21}$

\section{Shortcomings and strong points of the study}

We did not measure willingness to cooperate or attitudes towards delirium, which, in hindsight, might have given us more insight into why participants attended the educational sessions, and how well the learnt material is implemented in practice. Another important aspect to consider is the planning of the educational sessions. To facilitate participation of the nursing staff, we left it to each unit leader to plan the educational sessions. The staff members on unit B were free to attend the session if they wanted, though it was made clear that participation was greatly appreciated; the unit leader at unit A planned the educational session during a mandatory staff meeting, which had as a consequence that the educational session was also mandatory. These differences may have influenced the results of our study. Lastly, although we collected data from three different time points pre-test, we collected data for just one time point post-test. This was done for practical reasons, but it limits the conclusions we can make, as it might be possible that any differences we see after the intervention might not have consolidated on the long term, or the effect may have been the result of another external factor. We do not know, therefore, if the proportion and quality of the screenings remained high several months after the educational intervention had taken place.

Our study also has several strong points. First, our primary outcomes were changes in clinical practice, instead of knowledge as assessed by the answers on a questionnaire. Rather, we used the questionnaires as a tool in our educational intervention: the content and focus of the educational session was based on the outcomes of the first assessment with the questionnaires and in the second round the questionnaires were used to reinforce the knowledge learned during the educational sessions. Another reason we decided not to include the questionnaires as a main outcome is that it cannot be guaranteed that the questionnaires were completed independently and without external help, rendering them unreliable as a study outcome. A second strong point in our methodology is the extended pre-test data collection: data was collected for three time points prior to the introduction of the educational intervention, so as to create a good baseline and avoid unjustly 
assigning any changes in clinical practice to the educational intervention when there might have been a pre-existing trend towards more screening or geriatric assessments. Moreover, because of the retrospective data collection, the nursing staff was not aware of the study during any of the pre-test time points, thus avoiding the possibility that they might have been triggered to perform better on any of the main outcomes.

\section{Conclusion}

Overall, the educational intervention showed to have increased both the proportion of patients who were screened for delirium using the DOS as well as the quality of these screenings. Moreover, a trend, though not significant, was observed for the proportion of patients who received a geriatric consultation from a GNP. The intervention was generally considered as positive and useful by the participating staff members. The educational sessions are easy to tailor, as there is a comprehensive base-presentation which can be adapted to meet the needs of the different hospital units. Although the intervention comprises several steps, it is feasible for hospital-wide implementation as there is only one component (the educational session) which requires the physical presence of the participants; the other components (questionnaires, posters, flyers) can be completed or consulted individually. Further research with a longer follow up time and larger sample is needed to establish if the results are just temporary or consolidate over time. Ideally, the educational programme should not only focus on the nursing staff, but also on physicians, as they have the final say about a patients' treatment. Finally, unit leaders in hospitals should encourage the implementation of the learned material for optimal results. 


\section{References}

1. Inouye SK, Westendorp RGJ,Saczynski JS: Delirium in elderly people. Lancet 2014; 383:911-922

2. van Velthuijsen EL, Zwakhalen SM, Mulder WJ, et al: Detection and management of hyperactive and hypoactive delirium in older patients during hospitalization: a retrospective cohort study evaluating daily practice. Int J Geriatr Psychiatry 2017;

3. Inouye SK, Foreman MD, Mion LC, et al: Nurses' recognition of delirium and its symptoms: comparison of nurse and researcher ratings. Arch Intern Med 2001; 161:2467-2473

4. Steis MR,Fick DM: Are nurses recognizing delirium? A systematic review. J Gerontol Nurs 2008; 34:40-48

5. Teodorczuk A, Mukaetova-Ladinska E, Corbett S, et al: Reconceptualizing models of delirium education: findings of a Grounded Theory study. Int Psychogeriatr 2013; 25:645-655

6. Akechi $\mathrm{T}$, Ishiguro $\mathrm{C}$, Okuyama $\mathrm{T}$, et al: Delirium training program for nurses. Psychosomatics 2010; 51:106-111

7. American Psychiatric Association: Diagnostic and Statistical Manual of Mental Disorders (4th ed.), 4. Washington, DC, American Psychiatric Association, 2000

8. Yanamadala $M$, Wieland $D$, Heflin $M T$ : Educational interventions to improve recognition of delirium: a systematic review. J Am Geriatr Soc 2013; 61:1983-1993

9. van de Steeg $L, R$ IJ, Wagner $C$, et al: The effect of an e-learning course on nursing staff's knowledge of delirium: a before-and-after study. BMC Med Educ 2015; 15:12

10. Detroyer E, Dobbels F, Debonnaire D, et al: The effect of an interactive delirium elearning tool on healthcare workers' delirium recognition, knowledge and strain in caring for delirious patients: a pilot pre-test/post-test study. BMC Med Educ 2016; 16:17

11. Green LW, Kreuter MW, Deeds SG, et al: Health education planning: a diagnostic approach, Mountain View, CA, Mayfield Publishing Company, 1980

12. Milisen K, Cremers S, Foreman MD, et al: The Strain of Care for Delirium Index: a new instrument to assess nurses' strain in caring for patients with delirium. Int J Nurs Stud 2004; 41:775-783

13. Schuurmans MJ, Shortridge-Baggett LM,Duursma SA: The Delirium Observation Screening Scale: a screening instrument for delirium. Res Theory Nurs Pract 2003; 17:31-50

14. van de Steeg L, R IJ, Langelaan M, et al: Can an e-learning course improve nursing care for older people at risk of delirium: a stepped wedge cluster randomised trial. BMC geriatrics 2014; 14:69

15. Tauro R: Delirium awareness - Improving recognition and management through education and use of a care pathway. BMJ Qual Improv Rep 2014; 2:

16. Wand AP, Thoo $W$, Sciuriaga $H$, et al: $A$ multifaceted educational intervention to prevent delirium in older inpatients: a before and after study. Int J Nurs Stud 2014; 51:974-982 
17. Sockalingam S, Tan A, Hawa R, et al: Interprofessional education for delirium care: a systematic review. J Interprof Care 2014; 28:345-351

18. Siddiqi N, Harrison JK, Clegg A, et al: Interventions for preventing delirium in hospitalised non-ICU patients. Cochrane Db Syst Rev 2016; 3:CD005563

19. Teodorczuk A, Welfare M, Corbett S, et al: Developing effective educational approaches for Liaison Old Age Psychiatry teams: a literature review of the learning needs of hospital staff in relation to managing the confused older patient. Int Psychogeriatr 2010; 22:874-885

20. van den Boogaard $M$, Pickkers $P$, van der Hoeven $H$, et al: Implementation of a delirium assessment tool in the ICU can influence haloperidol use. Crit Care 2009; 13:R131

21. Inouye SK, Baker DI, Fugal P, et al: Dissemination of the hospital elder life program: implementation, adaptation, and successes. J Am Geriatr Soc 2006; 54:1492-1499 
I said nothing for a long time, just ran my fingertips along the edge of the human-shaped emptiness

that had been left inside me.

- Haruki Murakami (Blind willow, sleeping woman, 2006)

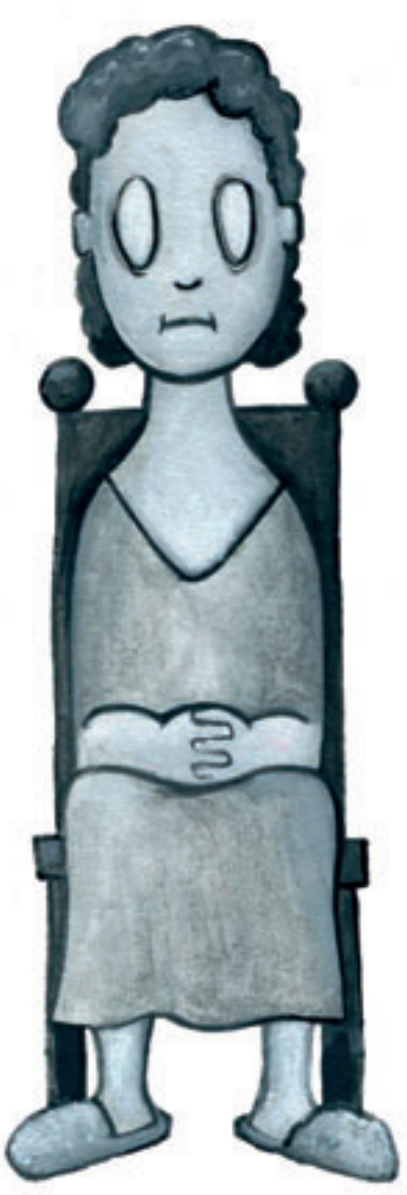




\section{CHAPTER 6 Managing hypoactive delirium: should or should we not use antipsychotics?}

This chapter has been accepted for publication in the Nederlands Tijdschrift voor Geneeskunde as:

Eveline L van Velthuijsen, Sandra MG Zwakhalen, Gertrudis IJM Kempen and Frans RJ Verhey. Behandeling van stil delier: wel of geen antipsychotica?

The article was translated from Dutch into English for the dissertation. 


\begin{abstract}
Delirium occurs in about 29 to $64 \%$ of all older patients in hospital. The majority of these patients develop a hypoactive delirium. Regarding the effectivity of using antipsychotic medication (mainly haloperidol) for the treatment of a hypoactive delirium, there is a lack of consensus: the guidelines of the Dutch society for General Practitioners (Nederlands HuisartsenGenootschap, NHG) and the guidelines of the Dutch society for Clinical Geriatrics (Nederlandse Vereniging voor Klinische Geriatrie) differ in their advice regarding this topic. Research has shown that antipsychotic medication can indeed be effective when used to minimise psychomotor agitation or problematic behaviour associated with hyperactive delirium. However, these symptoms are rarely present in patients with a hypoactive delirium. Antipsychotic have not been proven to be effective in reducing the duration of a delirium, do not decrease the severity of a delirium nor do antipsychotics decrease the length of hospital stay for delirious patients. In conclusion, taking into account the lack of evidence to support this practice, the use of antipsychotic medication to manage a hypoactive delirium in older hospitalised patients is not advised.
\end{abstract}




\section{Case}

An 83-year old woman is brought in to the Emergency Department. She had a broken hip due to a fall, and needs surgery. Postoperatively the woman is quiet and withdrawn, and she eats and drinks poorly. Her husband informs the medical staff that his wife is more quiet than usual and occasionally speaks gibberish. He does not recognise his wife like this. A clinical investigation confirms the woman is disoriented in time, place and person, and after conferring with a geriatrician, the woman is diagnosed with hypoactive delirium. Additional physical investigation reveals a urinary tract infection. The infection is treated with antibiotics. Should the woman also receive antipsychotic medication to help treat the symptoms of a hypoactive delirium?

Delirium is characterised by an acute change in consciousness, a decline in cognitive functions and the inability to focus, maintain or shift attention. ${ }^{1}$ Delirium is often accompanied by psychomotor disturbances (agitation or apathy) and psychotic symptoms. The symptoms of delirium fluctuate over the course the day. The psychomotor symptoms of delirium form the basis of the different subtypes, of which the hypoactive and hyperactive are the two main subtypes. The hypoactive delirium is characterised by, among others, psychomotor retardation, apathy and a slowing of speech. It is also quite common for delirious patients to fluctuate between the hypoactive and hyperactive subtype; this is referred to as a mixed delirium. There is a lot of debate regarding the effectiveness and safety of antipsychotics for the treatment of delirium in older patients, especially those with a hypoactive delirium. This debate affects a substantial group of patients: between 29 and $64 \%$ of older patients develop a delirium while in hospital, of which the majority constitutes a hypoactive delirium. ${ }^{2}$

\section{Guidelines}

There are several different national and international guidelines available for the treatment of delirium. In the Netherlands two guidelines are available: the "multidisciplinary guideline" published in $2013^{3}$ and the guideline published by the Dutch Society of General Practitioners (Nederlands HuisartsenGenootschap, NHG) in 2014. ${ }^{4}$ These Dutch guidelines concur with the guidelines of the English National Institute for Health and Care Excellence (NICE) and the guideline of the American Psychiatric Association (APA) on most topics (for example regarding nonpharmacological interventions such as a medication review, sleep hygiene protocols, nutrition and hydration). However, the Dutch multidisciplinary guideline deviates from the NHG, NICE and APA guidelines regarding the pharmacological management of delirium. The latter three guidelines advise caution in the use of antipsychotic medication (mainly haloperidol), and this should only be used after all non-pharmacological interventions have been employed without the desired 
effect, and if a patient is in distress, is agitated, or poses a threat to themselves or others. ${ }^{5,6}$ The Dutch multidisciplinary guideline is less strict regarding the use of antipsychotic medication, suggesting that anxiety as a result of the psychotic symptoms in hypoactive delirium are also a target for antipsychotic treatment. $3,7,8$ However, international experts advocate to restrict the use of antipsychotic medication to patients with behavioural problems and severe distress as a results of hallucinations or delusions, and only if de-escalation techniques have been tried. ${ }^{2,9-12}$

The current literature review provides an overview of the efficacy and safety of the use of antipsychotic medication for hypoactive delirium in older hospitalised patients. The following research question was formed: What is the efficacy of antipsychotic medication for treating hypoactive delirium in older hospitalised patients?

\section{In-depth information}

The exact pathophysiology of delirium remains unknown, though there are some neurobiological mechanisms which are known to play a role in developing or maintaining a delirium. These include a disruption of neurotransmission (especially an increase in dopamine and a decrease in acetylcholine), neuro-inflammation, metabolic disruptions, electrolyte disruptions and genetic factors. ${ }^{2}$ However, it remains uncertain if and how these different neurobiological factors play a role in the development of the different subtypes of delirium and the psychotic symptoms that often go with a delirium.

The function of antipsychotic medication, especially haloperidol, for delirium is based on its effectiveness on psychotic symptoms in people with psychiatric problems. Haloperidol is a strong dopamine antagonist and is used mainly to combat the excess dopamine present in delirium. ${ }^{2}$ Beside the excess in dopamine, a deficiency in acetylcholine is often seen in delirious patients. Haloperidol, in addition to its antagonistic effects on dopamine, also has an anticholinergic function, which could have a negative impact on the already decreasing levels of acetylcholine. Medication with an anticholinergic effect are therefore often counterindicated for delirious patients. ${ }^{3}$

\section{Literature search}

To answer the abovementioned question, we used five acclaimed international systematic reviews on the topic. ${ }^{2,9-12}$ These five reviews were published between (and including) 2014 and 2017 and contain the majority of the international scientific publications regarding the pharmacological treatment of delirium up until March 2017. We conducted an additional search for the period between 1 January 2017 and 30 November 2017 to include any relevant articles that might have been published after the last review was performed. This search strategy resulted in an 
additional three articles. ${ }^{13-15}$ This means that in total we included five reviews and three original research articles with primary data. An overview of the search strategy can be found in Figure 1.

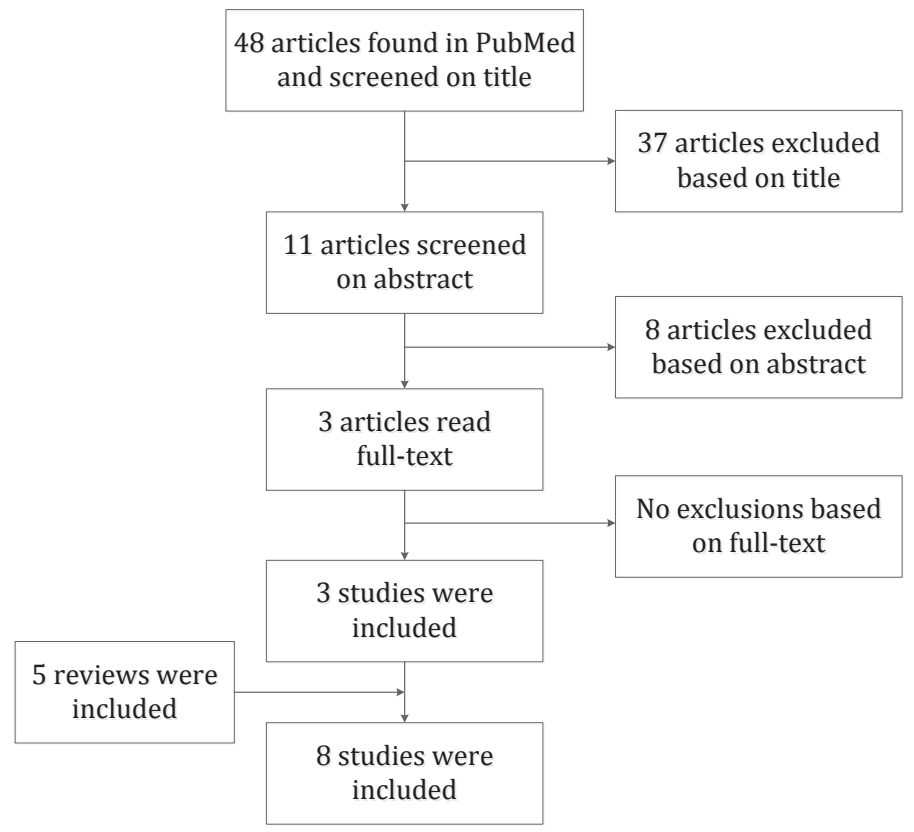

Figure 1. Flowchart of the inclusion process

Table 1. Overview of the effects of antipsychotics for the treatment of delirium in older hospitalised patients

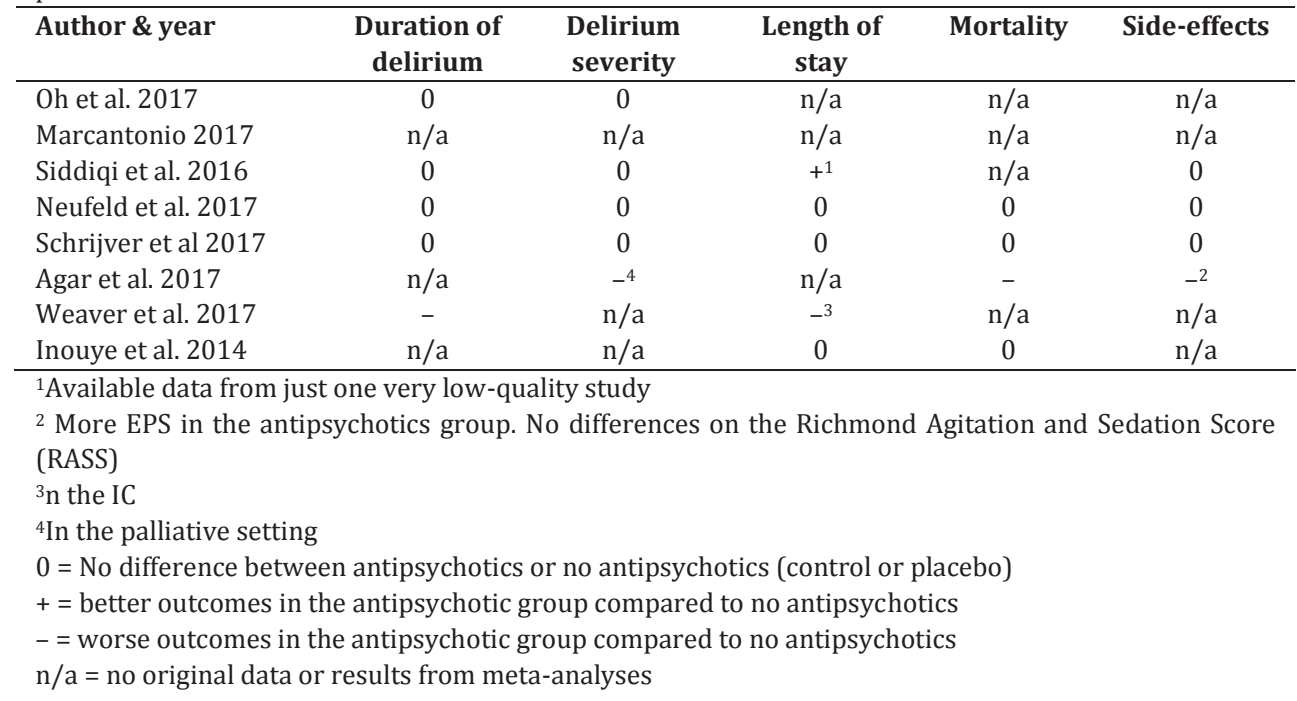




\section{Duration of the delirium}

Five studies assessed the duration of the delirium (in days) as a treatment outcome.10-12,14,15 Four out of these five studies did not find any differences in the duration of delirium between patients who were and were not treated using antipsychotics. ${ }^{10-12,14}$ One study found that delirious patients who were treated using antipsychotics had significantly longer delirious episodes compared to patients who did not receive antipsychotics. ${ }^{15}$

\section{Delirium severity}

Five articles mention delirium severity as a treatment outcome.10-14 Four out of these five articles do not find any significant differences in the severity of the delirium symptoms between the antipsychotic and non-antipsychotic patients groups. ${ }^{10-12,14}$ One study conducted in the palliative setting found that patients who received antipsychotic medication to treat the delirium had more and more severe delirium symptoms compared with patients who did not receive antipsychotics to treat the delirium. ${ }^{13}$ This study was also the only study to look into delirium symptoms and patient distress. They found that patients who receive antipsychotic medication for the treatment of delirium experienced significantly more hallucinations and more problems with behaviour and communication than patients who received a placebo.

\section{Length of hospital stay}

Length of hospital stay was reported in five of the eight included articles. ${ }^{2,11,12,14,15}$ In three of these, no significant differences in length of stay between patients who did and did not receive antipsychotic medication for the treatment of delirium were found. ${ }^{2,12,14}$ One study, conducted in the intensive care, found that delirious patients who received antipsychotics were in the IC significantly longer than patients who did not receive antipsychotics. ${ }^{15}$ One systematic review reported on the length of stay, and found a positive effect of antipsychotics on length of stay (i.e. patients who received antipsychotics had a shorter length of hospital stay). However, these results were based on a single, very low quality study due to serious imprecision in the results. ${ }^{11}$

\section{Mortality}

Mortality as a result of delirium is discussed in four of the eight articles included in this review,2,12-14 but the results are not conclusive. Three articles (two reviews and a recent large-scale Dutch study) found no differences in mortality between patient groups who did and did not receive antipsychotic medication for the treatment of delirium. 2,12,14 Another large-scale Australian study, however, did find significantly higher mortality rates in the patient group who were treated using antipsychotics. ${ }^{13}$ 


\section{Side-effects of antipsychotic medication}

Four articles mention the possible side-effects of antipsychotic medication for delirium. ${ }^{11-14}$ Three out of these four articles did not find a significantly higher prevalence of possible side-effects (such as prolonged QT-times or EPS) among patients treated with antipsychotic medication.11,12,14 One study found a higher incidence of EPS among patients who received antipsychotic medication for the treatment of delirium, but these were mild symptoms. ${ }^{13}$ This same study also found that mortality was higher among patients who were treated with haloperidol versus patients who received a placebo. A third group of patients in this study received risperidone to treat the delirium, but there was no increased chance of mortality in this group.

\section{Discussion}

We analysed the outcomes of different studies and reviews regarding the effectiveness of antipsychotics in the treatment of hypoactive delirium in older hospitalised patients. Evidence of effectiveness was not found for any of the treatment outcomes (duration of delirium, delirium severity, mortality, length of stay and side-effects) for delirium in general. We should bear in mind that agitation and behavioural problems were not found to be a treatment outcome in any of the studies, but also that these symptoms very rarely occur in hypoactive delirium. Another important aspect to consider when interpreting the results is that no studies were performed where the distinction between the different delirium subtypes was made: instead, all studies included delirious patients in general, without considering subtype.

One of the included reviews described one study in which a shorter length of stay was observed for patients who received antipsychotics for their delirium. However, the authors of the review assessed the quality of this study as being very low. ${ }^{11}$ Another study, in a randomised double blind trial, found that the length of hospital stay did not differ between the treatment and control group. This same study found, however, that patients in the antipsychotic group had more severe symptoms, suffered more distress, had a 1,5 higher chance of mortality and suffered EPS more often than patients who had received a placebo. ${ }^{13}$

Antipsychotic may be effective in reducing agitation and behavioural problems. However, based on the results of the studies described in our review, antipsychotics do not have a beneficial effect on the duration or severity of a delirium, the distress experienced by delirious patients, or the length of hospital stay. Moreover, the use of antipsychotic medication increases the chances of negative side-effects, compared to placebo or non-pharmacological interventions. The side-effects found for antipsychotics seem to be mild, however.

A common argument used for the treatment of hypoactive delirium with antipsychotics is that the medication might alleviate the distress patients with a 
hypoactive delirium experience as a result of hallucinations or delusions. ${ }^{9}$ However, one of the studies described in our review found that in the palliative setting, delirious patients (without differentiating subtypes), who were treated with antipsychotic medication suffered more distress than patient who received a placebo. ${ }^{13}$ A qualitative study from 2009 supports the finding that antipsychotic medication does not alleviate distress in delirious patients: they found that the majority of the patients who were able to recall the delirious episode experienced distress, despite the use of antipsychotic medication. ${ }^{16}$

Delirium is a complex, multifactorial disorder that is common among older hospitalised patient, and which knows several subtypes. Nevertheless, in the research concerning the treatment of delirium, these different subtypes are seldom taken into account: all types of delirium are considered, researched and treated as a unitary syndrome. When interpreting the results from our review, it is important to bear in mind that none of the included studies differentiated between the different subtypes. However, when treating a disorder, it is paramount to consider what is being treated and why. The results of our research show that antipsychotic medication does not have a beneficial impact on the duration or severity of delirium, or the length of hospital stay. Patients were also not less distressed if their delirium was treated with antipsychotic medication.

\section{Conclusions and implications for practice}

The main question of this study was: what is the efficacy of antipsychotic medication for treating hypoactive delirium in older hospitalised patients? Based on the results we can conclude that there is not enough evidence to support the use of antipsychotic medication for the treatment of hypoactive delirium in older hospitalised patients. A delirium does not become shorter or less severe, it does not decrease the length of hospital stay, and patients seem to be distressed by a delirium whether or not they are treated with antipsychotic medication. As of yet, no studies have been performed specifically on the treatment of hypoactive delirium, and the results in our study are, therefore, based on the results of studies with delirium in general, without specifying subtypes. However, patients with a hypoactive delirium seldom have behavioural problems or agitation, and will rarely pose a threat to themselves or others. As such, we have reached the (temporary) conclusion that the evidence for the use of antipsychotics for hypoactive delirium are lacking. Based on this conclusion, the advice that hypoactive delirium should be treated with antipsychotic medication cannot be supported. Future research should focus on performing randomised, double-blind, adequately powered studies into the treatment of delirium, where the different subtypes are differentiated and taken into account. 


\section{References}

1. American Psychiatric Association: Diagnostic and Statistical Manual of Mental Disorders (4th ed.), 4. Washington, DC, American Psychiatric Association, 2000

2. Inouye SK, Westendorp RGJ, Saczynski JS: Delirium in elderly people. Lancet 2014; 383:911-922

3. Nederlandse Vereniging voor Klinische Geriatrie: Richtlijn Delier Volwassenen. 2013;

4. Nederlands HuisartsenGenootschap: NHG - Standaard delier, 2014

5. National Institute for Care and Health Excellence: Delirium: prevention, diagnosis and management (NICE guideline CG103), 2010

6. American Psychiatric Association: Practice guidelines for the treatment of patients with delirium, 2010

7. Leentjens AF, Molag ML, Van Munster BC, et al: Changing perspectives on delirium care: the new Dutch guideline on delirium. J Psychosom Res 2014; 77:240-241

8. Meagher D, Agar MR, Teodorczuk A: Debate article: Antipsychotic medications are clinically useful for the treatment of delirium. Int J Geriatr Psychiatry 2017;

9. Marcantonio ER: Delirium in Hospitalized Older Adults. N Engl J Med 2017; 377:1456-1466

10. Oh ES, Fong TG, Hshieh TT, et al: Delirium in Older Persons: Advances in Diagnosis and Treatment. JAMA 2017; 318:1161-1174

11. Siddiqi N, Harrison JK, Clegg A, et al: Interventions for preventing delirium in hospitalised non-ICU patients. Cochrane Db Syst Rev 2016; 3:CD005563

12. Neufeld KJ, Yue J, Robinson TN, et al: Antipsychotic Medication for Prevention and Treatment of Delirium in Hospitalized Adults: A Systematic Review and MetaAnalysis. J Am Geriatr Soc 2016;

13. Agar MR, Lawlor PG, Quinn S, et al: Efficacy of Oral Risperidone, Haloperidol, or Placebo for Symptoms of Delirium Among Patients in Palliative Care: A Randomized Clinical Trial. JAMA Intern Med 2017; 177:34-42

14. Schrijver EJM, de Vries OJ, van de Ven PM, et al: Haloperidol versus placebo for delirium prevention in acutely hospitalised older at risk patients: a multi-centre double-blind randomised controlled clinical trial. Age Ageing 2017; 1-8

15. Weaver CB, Kane-Gill SL, Gunn SR, et al: A retrospective analysis of the effectiveness of antipsychotics in the treatment of ICU delirium. J Crit Care 2017; 41:234-239

16. Bruera E, Bush SH, Willey J, et al: Impact of delirium and recall on the level of distress in patients with advanced cancer and their family caregivers. Cancer 2009; 115:2004-2012 
Begin at the beginning, and go on till you reach the end.

Then stop.

- Lewis Carroll (Alice in Wonderland, 1856)

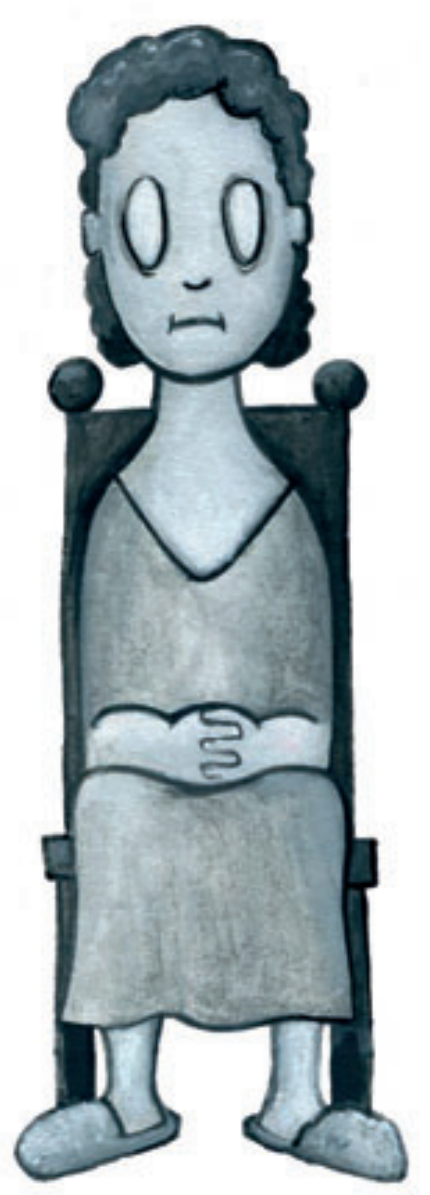


CHAPTER 7

General discussion 


\section{Introduction}

Delirium is a very common and serious complication of hospitalisation among older patients and can have severe short- and long-term consequences for the patient. No efficacious cure -either pharmacological or non-pharmacological- exists for delirium, but guidelines exist nationally and internationally to optimise recognition and management of delirium. This dissertation had one main theme-delirium in older hospitalised patients-, which can be divided into two sub-themes: recognition and management. This general discussion presents and discusses the main findings of this dissertation, as well as several methodological strengths and limitations of the studies, and implications for clinical practice and future research.

\section{Main findings}

Screening for delirium in daily hospital practice is best done using the Nursing Delirium Screening scale (NuDESC). The Delirium Observation Screening (DOS) score, which is the standard delirium-screening instrument used in the Maastricht University Medical Centre+ (MUMC+), was also found to be a feasible and good instrument for screening for delirium, and can be used to recognise both hyperactive and hypoactive delirium. Despite the use of the DOS in the MUMC+, however, we found that delirium among older patients is still severely underrecognised and under-reported in daily hospital practice. Especially hypoactive delirium, which is the most common subtype among older delirious patients, was probably often missed or misdiagnosed by the medical and nursing staff. Educating the nursing staff on the importance of delirium in older patients, and explaining how the DOS should be used, resulted in an increase of the percentage of patients who were screened using the DOS, and increased the quality of the screenings. With regard to the management of delirium, we found that, contrary to the guidelines, almost $90 \%$ of the delirious patients received antipsychotic medication (mostly haloperidol) to manage the delirium. Dutch and international guidelines on the management of delirium in hospitalised patients state that antipsychotic medication should be used only after non-pharmacological interventions have been tried without the desired results. Moreover, patients should be distressed or pose a danger to themselves or others as a result of psychomotor agitation or behavioural problems. We found that there is little to no evidence to warrant the use of antipsychotic medication for the management of symptoms for patients who suffer a hypoactive delirium. Instead, non-pharmacological interventions should always be the first and main step in delirium management, although our study pointed out that less than $70 \%$ of delirious patients received non-pharmacological interventions. One of these non-pharmacological interventions is a medication review. We found that a medication review can significantly shorten a delirious episode for some patients. Moreover, providing delirium education to the nursing 
staff tended to increase geriatric consultations and an increase in medication reviews for delirious patients.

\section{Reflection}

This section reflects on the main results of this dissertation. First, a reflection on recognition and reporting of delirium and delirium subtypes in older hospitalised patients in daily hospital practice is given. Second, the management and guideline adherence for delirium in older hospitalised patients is discussed.

\section{Diagnosis, subtypes and reporting}

This dissertation provided more insight into what the best screening instruments for delirium in older hospitalised patients are, how well delirium and its subtypes are recognised, and whether delirium is reported in a patient's medical file. The results showed that there is a wide variety of good and feasible instruments for the recognition of delirium: more than 40 different instruments were available at the time our study was conducted, and more are published every year. A critical review of the psychometric properties and feasibility of these instruments can provide valuable information for health care providers seeking an instrument to detect delirium. We found that although some instruments have good psychometric properties, not all are feasible for use in daily hospital practice. The CAM, for example, has high sensitivity and specificity, but is not very feasible, as it requires a trained specialist and can be time consuming. The DOS, which is used in the MUMC+, seems to be a good and feasible instrument as well.

Screening for a disorder requires that patients who are at risk and have not yet developed the disorder are screened regularly to assess any tell-tale signs of the early stages of the disorder. For delirium this means that all older hospitalised patients at risk for delirium should be screened continuously to monitor the patient. Although the DOS is a good instrument for screening for delirium in older hospitalised patients, its feasibility is somewhat limited as it requires to be completed three times a day (once during every nursing shift) to be reliable in practice.

Moreover, in the MUMC+, the DOS is mostly used to monitor the severity of the delirium after a delirium is fully developed, instead of using it as a screening tool to identify early stage delirium or sub-syndromal delirium. This failure to screen (all) at-risk patients for delirium could explain why delirium in this patient group seems to be still severely under-recognised, especially the hypoactive subtype. Educating the nursing staff about delirium resulted in higher percentages of patients who were screened for delirium, and improved the quality of the screening.

This dissertation showed that delirium was diagnosed and reported for just $5 \%$ of the older patients admitted to the hospital, even though previous research has shown that the 'true' prevalence rates of delirium are estimated between $19 \%$ and 
$64 \%$ for people 65 years and older. ${ }^{1}$ Previous research has also found that up to $56 \%$ of all delirious episodes are of the hypoactive subtype. ${ }^{2}$ The results in this dissertation, however, showed that just $23 \%$ of the diagnosed delirious episodes were of the hypoactive subtype, indicating that the vast majority of the missed or unrecognised deliriums in daily practice are probably of the hypoactive subtype. Patients with hyperactive delirium can be disruptive and have a negative impact on a hospital ward, making work difficult for the health care workers and can place significant burden on family members visiting their delirious relative. However, this hyperactive behaviour has a protective feature as well: it promotes recognition and treatment - stopping problematic behaviour is beneficial for all involved parties. The hypoactive subtype, however, is a different story: these patients mostly do not show any behavioural problems and are often quiet and withdrawn, not creating any problems or attracting any attention to themselves. The severe underrecognition of hypoactive delirium is probably (among others) due to this lack of behavioural problems.

The three main findings with respect to diagnosis, subtype detection and reporting of delirium among older hospitalised patients as mentioned in this paragraph are: there are plenty of good and fairly feasible diagnostic tools to detect delirium; that despite this availability, there is severe under-recognition of delirium (particularly hypoactive) in daily practice; and that education for the nursing staff increases the percentage of patients who are screened for delirium. Taking these results into account, it may be argued that the recognition of delirium will not be helped any further by developing more instruments. Instead, education of the staff and providing explanations and information to patients and family members might be much more effective than any further instrument development: indeed, even the best instruments could prove ineffective in the hands of untrained professionals.

\section{Guideline adherence and delirium management}

There are guidelines on how to manage delirium in hospitalised patients, both nationally and internationally. The most well-known guidelines, on which the national guidelines in most other countries are based, are the guidelines from the American Psychiatric Association (APA) in the USA and the National Institute for Health and Care Excellence (NICE) in the United Kingdom. The Dutch guidelines are also mostly based on these two. According to the APA, NICE and Dutch guidelines, the first step in managing a delirium is always to find and treat the underlying cause of the delirium (e.g. an infection or dehydration). Step two includes an array of non-pharmacological nursing interventions aimed at reorientation (e.g. placing a clock and calendar in the patients' room) and the optimisation of patient care (e.g. mobilisation, nutrition, and optimising a patients' medication).

The APA and NICE guidelines differ from the Dutch guidelines on the pharmacological management of delirium. According to the APA and NICE guidelines, medication (mostly antipsychotics, specifically haloperidol) should be 
considered only after all the non-pharmacological measures have been taken, patients are still very delirious, posing a danger to themselves and/or others or are in severe distress. ${ }^{3}$ They emphasise caution when a patient does not exhibit and psychomotor or behavioural problems. The Dutch guidelines are less stringent on this issue, and propose that the hypoactive symptoms could be treated with antipsychotic medication. ${ }^{4,5}$

This dissertation provided more insight into how delirium is being managed in daily practice, and to which extent practice is consistent with the guidelines. We also assessed the effects of a medication review on different aspects of delirium, assessed the effects of nursing education on certain aspects of delirium management, and looked deeper into whether or not antipsychotic medication should be used for the treatment of hypoactive delirium (the major difference between the international and Dutch guidelines). The results showed that the main technique used to manage a delirium is the administration of antipsychotic medication, contrary to the advice of the guidelines. Moreover, there is very little and circumstantial evidence for the effectiveness of the use of antipsychotic medication in the treatment of hypoactive delirium. Additionally, performing a medication review for patients who are delirious could result in shorter delirious episodes, and nursing education may result in more geriatric consultations.

From the results of our studies, it can be concluded that guideline adherence in the management of delirium is rather poor. We found that about $86 \%$ of delirious patients are treated with antipsychotic medication (especially haloperidol), while just $69 \%$ of delirious patients received the non-pharmacological interventions described in the guidelines. Ideally, this should be $100 \%$. Moreover, only about twothirds of the delirious patients received a geriatric consultation. Considering this overall poor guideline adherence, it could be argued that it is in the patients' benefit to request a geriatric consultation for all delirious patients. However, this may not be feasible in daily practice, considering the high number of older hospitalised patients who potentially develop a delirium. Educating non-geriatric staff on the importance of delirium may be more feasible. The results from chapter five show that delirium education for nurses slightly increases the percentage of patients receiving a geriatric consultation. This may mean that education could increase awareness for delirium and the sense of urgency when treating older patients with delirium, which might in turn lead to a possible increase in guideline adherence.

One specific advice from the guidelines states that delirious patients should receive a medication review, restricting medication to the minimum amount of essential medications (e.g. by lowering the dose, cancelling non-essential medication or replacing some medications with other, less deliriogenic alternatives). ${ }^{6}$ At the MUMC+ such a medication review is performed for all patients who receive a geriatric consultation. This medication review, performed by a geriatrician and a clinical pharmacist, is described and assessed in chapter four of this dissertation. We found that patients taking between zero and six medications who received a medication review had substantial shorter delirious episodes than comparable 
patients who did not receive a medication review. Patients taking between seven and eleven medications also had slightly shorter delirious episodes when they received a medication review. The duration and severity of a delirium are inversely correlated with the success of functional recovery of patients (i.e. the shorter the delirium, the better the recovery). ${ }^{7}$ Moreover, polypharmacy is an important precipitating factor delirium in older hospitalised patients. ${ }^{1}$ Preventing polypharmacy at the beginning of a hospitalisation, by means of a medication review, could therefore not only shorten a delirious episode, but also minimise the risk of developing a delirium. ${ }^{2}$

Antipsychotic medication is proposed by the guidelines as a last resort, to be used sparingly after all else failed and the patient remains agitated or distressed. However, we found that it is the most widely and commonly used "treatment" for delirium in daily practice. To date, as far as we know no well-designed and properly powered studies have been carried out which confirm the effects of antipsychotic medication for the treatment of prevention of delirium. ${ }^{8,9}$ While antipsychotic medication (mostly haloperidol) has shown to be effective in subduing the behavioural problems and psychomotor symptoms in patients who are severely agitated and who may pose a danger to themselves or others, this should not be the main or only component of delirium management. This is especially true for the hypoactive subtype, as those patients do not have any behavioural problems or psychomotor agitation. ${ }^{2}$

In conclusion, it could be argued that guideline adherence is rather poor: reorientation strategies are not implemented for $100 \%$ of delirious patients and there is evidence that many patients may receive antipsychotic medication prematurely or unnecessarily, contrary to the guidelines. However, education can improve guideline adherence, thus improving delirium management. Moreover, one of the strategies for managing delirium (the medication review) was proven to be effective in shortening the delirious episode in some patients.

\section{Methodological considerations}

This section discusses several methodological strengths and limitations of the studies presented in this dissertation. The study design, study population and generalisability are discussed.

\section{Study design}

The main objective of this dissertation was to study delirium detection and management in daily hospital practice. The dissertation consists of five different studies, for which three different designs were used: two (systematic) literature reviews, two retrospective cohort studies, and one intervention study. Combining different methodologies when studying a specific subject can help to shed light on a topic from different angles and create a more complete picture. When studying 
recognition and management of a certain disorder in daily hospital practice, one must be careful not to disrupt or influence the status quo with the study design. Performing prospective studies such as randomised controlled trials (RCTs) can have many strong points: they allow the researcher to control for variables which may influence study outcomes, to perform blinded studies, and they often enable the researcher to infer causality. However, when assessing daily practice, prospective methods could potentially influence daily practice, because the presence of a researcher may prompt people to think about delirium more than they would normally do (like when you tell somebody not to think about pink elephants). Consequently, outcomes may be biased, generating a picture which is not in line with daily practice.

Gathering data retrospectively may paint a more realistic picture in this respect, as it evaluates regular care: at the moment the data was registered there was no researcher or study protocol present to influence the physician or nurse registering the data. However, it is important to keep in mind the research question: if you want to know the actual occurrence of delirium, prospective methods are better, but when you want to know how often delirium is being recognised in daily practice, retrospective methods may be preferred. An intervention study combined with retrospective data collection provides the opportunity to assess the effects of the intervention on daily practice. It is important that research questions match the research design, however. If we would have wanted to know what ('true') percentage of older patients developed a delirium during their hospital stay, the retrospective method would not have been appropriate.

However, our retrospective method also has a downside: the data that can be collected is limited to the data someone else has recorded. Moreover, this data was recorded for medical treatment reasons and not for research purposes, so no standardised research protocols are available, which may have limited the reliability of the data. For example: delirium is not always diagnosed based on a specific diagnostic instrument, but often on clinical appraisal, and different physicians may have (slightly) different diagnostic criteria. As mentioned above, retrospective methods are limited to the data available in the database, without being able to influence or decide what types of outcomes you want. For example, performing a follow-up study to see whether some patients are readmitted to the hospital or have a better functional recovery can be very challenging because a patient may be readmitted to a different hospital, and data on functional recovery after hospital discharge may not available. In the case of the evaluation of the medication review, the retrospective method did not allow us to study the possible preventive effects of the medication review, because all patients who received a medication review were already delirious. Moreover, we may have missed some patients with delirium because they were not reported in a discharge letter or because these patients did not receive a geriatric consultation.

However, in our studies, we took precautions to avoid as much as possible influence of external factors: patient identification and assessment of the duration 
of delirium was always done using the same methods and parameters, and there were no new guidelines published in the span of this dissertation.

\section{Study population}

This dissertation focussed mainly on older $(65+$ or $70+)$ patients who developed a delirium during hospital stay. Additionally, nurses caring for this patient population were included in one of the studies. Identification of the relevant patient files was done in several ways for the different studies. For the study population in chapter three, the relevant patient files were identified through all the delirium diagnoses made by the departments of geriatric and psychiatry, and by scanning all the discharge letters of patients aged 65 years or older on the presence of delirium, confusion or related words. In this way, we were able to identify most patients whose delirium diagnosis was recorded in the patient files. For the study of the medication review (chapter four), the files of all patients who had received a geriatric consultation and a medication review in the indicated time periods were scanned for a mention of delirium. The patient files included in chapter five (the educational intervention) were identified by going through the files of all the patients who were 70 years or older and had been admitted to the specific wards at the indicated time periods.

All the studies had in common that the delirium diagnosis was obtained from the patient files. Therefore, delirious patients could only be recognised as such if the diagnosis of delirium was actually recorded in the patient file. We know from our results in chapter three that there was a severe under-recognition of delirium, especially of the hypoactive subtype. Consequently, patients with a severe delirium or a delirium of the hyperactive subtype are probably over-represented in our samples and patients with a mild delirium or delirium of the hypoactive subtype are under-represented.

The over-representation of the hyperactive subtype in our samples could have affected our outcomes. Very little is known about the aetiology of delirium and the different subtypes. It is unclear why there are different subtypes of delirium, and what causes these different subtypes to manifest themselves in certain patients and not in others. It is known that patients with a hypoactive delirium have an overall poorer prognosis ${ }^{2}$, but it is unclear whether this is because of the delirium subtype, or because a poorer physique elicits a hypoactive delirium (what came first, the chicken or the egg?).

However, identifying patients through the diagnosis in the patient files fitted best with our research questions, as we wanted to know what the daily practice was regarding the recognition and management of delirium and guideline adherence. Reporting delirium in a patient files should be done for all delirious patients, so identifying the relevant files by whether or not the diagnosis is reported in a patient file provided us with the information we were seeking. 


\section{Generalisability}

The studies described in chapters three, four and five were all carried out at the MUMC+. The MUMC+ is an academic hospital based in the south of Limburg, which is the province with one of the highest percentages of older (65+) people in the Netherlands. Academic hospitals are generally larger than non-academic hospitals, and are more used to research and implementing best practice based on research outcomes. Being a hospital in the "oldest" province of the Netherlands can also mean that a higher percentage of older patients is admitted to the MUMC+ than to other (academic) hospitals in the Netherlands, also increasing the number of potentially delirious patients. However, results from our study performed in chapter three were in line with similar studies performed in general hospitals abroad,10,11 indicating that the percentage of patients who are diagnosed with delirium is similar in different countries and hospitals.

With regard to the implementation of the interventions described in chapters four and five (the medication review and the educational intervention), these specific interventions were fairly location-specific, as they were designed to be implemented in the MUMC+ with the resources available at the MUMC+. However, the results of these interventions can be generalised to other hospitals, both academic and non-academic. All older patients in a hospital run a heightened risk of developing a delirium, and the risk factors for developing a delirium are the same for these patients, no matter which hospital they are admitted to. Designing and implementing an intervention that modifies the presence of these risk factors (as with a medication review or educational intervention), will probably result in the same changes in the patients as we observed in our studies.

The main modifiable factor in these implementation studies is the 'culture' or attitude of healthcare professionals: it is important that healthcare professionals know and understand the importance of delirium in older hospitalised patients. If the attitude is not positive towards delirium, it may be more difficult to produce changes through interventions: nursing staff may not attend educational interventions or implement what they learned, and a medication review will not have any effect if the proposed changed in medication are not actually implemented. However, it may be assumed that the overall culture in hospitals leans toward wanting to improve care for all patients, thus implementing knowledge gained through research.

Delirium is also highly prevalent in nursing homes, ${ }^{12}$ and patients who suffered a delirium when in hospital run a higher risk of having to move to a nursing home. ${ }^{13}$ The results of this dissertation could partly also be generalised to this setting: educating nursing-home nurses might improve recognition and management of delirium in nursing home residents, and medication reviews might help shorten or even prevent a delirium. The results regarding the instruments for the detection of delirium could also be generalised to other settings: the NuDESC or CAM can be used in nursing homes, and some instruments were specifically designed for use by 
family members (such as the $\mathrm{I}_{-\mathrm{AGeD}^{14}}$ or FAM-CAM ${ }^{15}$ ), who could use these instruments to recognise a delirium in community-dwelling older people.

\section{Implications for practice}

The results of this dissertation have several implications for clinical practice. First, we found that delirium is severely under-recognised in daily hospital practice and that the implementation of delirium guidelines in practice is subpar. Medication seems to be the prime source of management, although no good evidence exists to warrant this practice, especially when treating the hypoactive subtype of delirium. Considering the severe negative effects associated with, especially hypoactive, delirium, hospitals should place much more emphasis on the importance of timely recognition and subsequent adequate management of delirium in old age. Moreover, $30-40 \%$ of all deliriums could be prevented if proper preventive measures are taken. ${ }^{16,17}$ However, medical and nursing staff generally has very high work pressure, and adding more tasks is not always possible. The Hospital Elder Life Program (HELP), developed by Inouye and colleagues has been implemented in many hospitals worldwide and has proven to be effective in significantly reducing delirium prevalence and severity, improved functional recovery and mobilisation, and decreased number of readmissions for older patients. In some cases the program has lowered work-related stress and pressure for the nursing staff; furthermore HELP has been proven to be cost-effective. ${ }^{18}$ The core components of the HELP programme are keeping older hospitalised patients oriented to their surroundings, for example by using a big calendar and a clock, and talking to patients about where they are and why; and providing patients with everything they need to get better as fast as possible: medication reviews, adequate nutrition, hydration, sleep and mobilisation. It also includes a big educational component. The programme has been proven to prevent delirium, falls and functional deterioration ${ }^{18}$. The HELP programme is most often implemented with the help of skilled volunteers. It generally takes between six and nine months to start up a HELP programme. In the Netherlands, HELP has been implemented in at least three academic and non-academic hospitals since 2015, and a Dutch implementation guide has been developed to aid other hospitals in the implementation of the HELP programme. Some of the components of the HELP programme are already implemented in the MUMC+: nursing education, a medication review, orientation strategies, etc. Moreover, there are many volunteers who already offer their help, who could potentially be trained to become a HELP volunteer. Thus, the MUMC+ already has some of the components of the HELP programme, and implementing HELP in the MUMC+ could be a great improvement in geriatric care, benefitting all parties involved.

Second, it should become mandatory to report delirium in the patient's file, as a previous delirium increases the chances of developing another one during subsequent hospitalisations. Reporting a delirium in the patient's file can therefore 
help to identify more patients at risk and subsequently prevent a new delirium. Moreover, considering the vulnerability and very high risk of developing a delirium in older hospitalised patients, especially post-surgery, delirium should be included in the list of possible complications and an information flyer should be provided to all older patients who undergo elective surgery. In doing so, patients and their family are better prepared for the possibility of a delirium and can provide preventive measures such as pictures from home, their own pillow or pyjama's. Moreover, family members can be a great source of information to help in the recognition of delirium. Reliable and valid instruments to detect delirium have been developed especially for family members, as family often know a patient well and are the first to notice a change in a patients' behaviour, which could be indicative of a delirium. Delirium can be a highly traumatic experience for both patients and their loved ones, ${ }^{19}$ and providing information on this disorder may help patients and family members to cope with the situation and subsequent feelings of shame. ${ }^{20}$ Another added benefit of informing patients of delirium prior to all elective surgery is that it may increase the awareness and sense of urgency of delirium among physicians and nurses.

Third, medication reviews have shown to decrease the duration of the delirium in a group of delirious patients. Evidence from previous studies also shows that polypharmacy is a precipitating factor for delirium, ${ }^{1}$ indicating that a medication review could possibly prevent delirium in older hospital patients. Knowing this, a thorough medication review, carried out by a geriatrician and/or clinical pharmacist should be performed for every at-risk patient admitted to hospital. Preferably, this should be done on the first day of the hospital admission to prevent any iatrogenic complications associated with polypharmacy.

Fourth, education has proven to increase the screening for delirium and to improve the quality of these screenings. These improvements became visible after a onehour educational session accompanied by informational posters. Increased emphasis on delirium in medical and nursing schools could have very beneficial effects on the detection and management of delirium. We therefore advise to increase the attention on delirium in the regular education programs of all health care professionals. This may refer to the guidelines and the timely recognition and management and the different types of delirium.

\section{Implications for future research}

Although delirium research has come a long way, especially in the last 20 years, there are still fields which need further studying. First, delirium research should veer away from developing and validating more diagnostic or screening instruments. The currently available instruments are sufficient to diagnose delirium: the problem does not lie with the instrument, but with those wielding it. Creating more instruments is a type of symptom management that cannot solve the issue at the heart of the problem: a lack of knowledge and sense of urgency when it 
comes to recognising and adequately managing delirium in older hospitalised patients. Instead, validating the already existing instruments, selecting the best ones and implementing these worldwide to standardise the diagnosis of delirium could be a much more powerful strategy.

Second, education has proven to be an effective tool in increasing the knowledge and guideline adherence for delirium. More research should be conducted in optimising delirium education and assessing the effects and cost-effectiveness of increasing the hours of delirium education in medical and nursing schools. Investing in education could potentially prevent a significant percentage of deliriums, decreasing healthcare costs and the burden for nursing staff. Moreover, providing short and comprehensive educational sessions for patients and family members could potentially prevent delirium or minimise the psychological impact of delirium on patients and family members, as the shock and trauma of a delirium would be less severe.

Third, evaluating the experiences of delirious patients, family members and healthcare professionals simultaneously could help to provide insight into what the people in these three groups might need to decrease the burden of a delirium. Would information about delirium help to cope with the situation? And is an informed family member more helpful in caring for a delirious patient compared to uninformed family? It can also be valuable to assess the effectiveness of several interventions used for the management of delirium. For example: how do physicians feel about prescribing antipsychotic medication for patients with a hypoactive delirium, how does a patient experience receiving this medication (does it make the distress less, worse or equal?) and how do family members feel about their family getting this medication? Do they notice a (positive) change? A qualitative study to answer these questions was also envisioned for this dissertation.

Fourth, there is evidence that suggests that the physical environment of a hospital may contribute to development of delirium, but also of other hospital-induced problems such as immobilisation. Does the use of colours to indicate different wards or hospital areas improve a patients' orientation? If a hospital encourages mobilisation by introducing enticing areas with activities, plants and art where patients can walk to, will this minimise immobilisation and help prevent delirium? These questions could be looked into with future research, and the results could be incorporated into the design of new hospitals.

Fifth, delirium research should focus on the aetiology, which still remains unclear. What are the exact underlying neurochemical processes that cause a delirium? If this question is answered, we might get one step closer to developing effective treatments for delirium. Moreover, delirium subtypes should be further looked into. There are currently four subtypes described in the literature (hyperactive, hypoactive, mixed, and no motor symptoms). ${ }^{21}$ It is unclear whether the different subtypes are different manifestations of the same condition, or are similar manifestations of different conditions, as the cognitive disturbances seem to be the 
same between all delirium subtypes, but the psychomotor manifestations can differ greatly. This difference is vital to further delirium research, because if we keep treating all subtypes of delirium as the same condition, we need to be absolutely sure that these subtypes have indeed the same underlying mechanisms.

Last, little is known about the prevalence and management of delirium in the community and in nursing homes. Older people are staying at home longer nowadays, before moving to a nursing home. This trend raises important questions regarding delirium in older, community-dwelling people: what is the prevalence of delirium in the community? How should family members and general practitioners manage these patients? Can steps be taken to prevent delirium in the community? Many of these questions also apply to the nursing home setting: what is the prevalence of delirium in the nursing home? How well is it being managed (i.e. guideline adherence)? Can it be prevented among a patient group with a very high prevalence of dementia and multimorbidity? The answers to these questions could potentially decrease delirium incidence and improve delirium care in primary, secondary and tertiary care (home, hospital and nursing home). 


\section{References}

1. Inouye SK, Westendorp RGJ, Saczynski JS: Delirium in elderly people. Lancet 2014; 383:911-922

2. Marcantonio ER: Delirium in Hospitalized Older Adults. N Engl J Med 2017; 377:1456-1466

3. National Institute for Care and Health Excellence: Delirium: prevention, diagnosis and management (NICE guideline CG103), 2010

4. Leentjens AF, Molag ML, Van Munster BC, et al: Changing perspectives on delirium care: the new Dutch guideline on delirium. J Psychosom Res 2014; 77:240-241

5. Meagher D, Agar MR, Teodorczuk A: Debate article: Antipsychotic medications are clinically useful for the treatment of delirium. Int J Geriatr Psychiatry 2017;

6. Van der Weele GM, Van Dijk A, Eekhof JAH, et al: NHG-Standaard Delier bij ouderen, in NHG-Standaarden voor de huisarts 2009Springer, 2009, pp 487-496

7. Speciale $S$, Bellelli $G$, Lucchi $E$, et al: Delirium and functional recovery in elderly patients. J Gerontol A Biol Sci Med Sci 2007; 62:107-108; author reply 108

8. Weaver CB, Kane-Gill SL, Gunn SR, et al: A retrospective analysis of the effectiveness of antipsychotics in the treatment of ICU delirium. Journal of critical care 2017; 41:234-239

9. Oh ES, Fong TG, Hshieh TT, et al: Delirium in Older Persons: Advances in Diagnosis and Treatment. JAMA 2017; 318:1161-1174

10. McCoy TH, Jr., Snapper L, Stern TA, et al: Underreporting of Delirium in Statewide Claims Data: Implications for Clinical Care and Predictive Modeling. Psychosomatics 2016;

11. Rooney S, Qadir M, Adamis D, et al: Diagnostic and treatment practices of delirium in a general hospital. Aging Clin Exp Res 2014; 26:625-633

12. Boorsma $\mathrm{M}$, Joling $\mathrm{KJ}$, Frijters $\mathrm{DH}$, et al: The prevalence, incidence and risk factors for delirium in Dutch nursing homes and residential care homes. Int J Geriatr Psychiatry 2012; 27:709-715

13. Witlox J, Eurelings LS, de Jonghe JF, et al: Delirium in elderly patients and the risk of postdischarge mortality, institutionalization, and dementia: a meta-analysis. JAMA 2010; 304:443-451

14. Rhodius-Meester HF, van Campen JP, Fung W, et al: [Development and validation of the Informant Assessment of Geriatric Delirium Scale (I-AGeD). Recognition of delirium in geriatric patients]. Tijdschr Gerontol Geriatr 2013; 44:206-214

15. Steis MR, Evans L, Hirschman KB, et al: Screening for delirium using family caregivers: convergent validity of the Family Confusion Assessment Method and interviewer-rated Confusion Assessment Method. J Am Geriatr Soc 2012; 60:21212126

16. Inouye SK, Bogardus ST, Charpentier PA, et al: A multicomponent intervention to prevent delirium in hospitalized older patients. N Engl J Med 1999; 340:669-676

17. Marcantonio ER, Flacker JM, Wright RJ, et al: Reducing delirium after hip fracture: a randomized trial. J Am Geriatr Soc 2001; 49:516-522 
18. Inouye SK, Baker DI, Fugal P, et al: Dissemination of the hospital elder life program: implementation, adaptation, and successes. J Am Geriatr Soc 2006; 54:1492-1499

19. Drews T, Franck M, Radtke FM, et al: Postoperative delirium is an independent risk factor for posttraumatic stress disorder in the elderly patient: a prospective observational study. Eur J Anaesthesiol 2015; 32:147-151

20. Pollard C, Fitzgerald M, Ford K: Delirium: the lived experience of older people who are delirious post-orthopaedic surgery. Int J Ment Health Nurs 2015; 24:213-221

21. Albrecht JS, Marcantonio ER, Roffey DM, et al: Stability of Postoperative Delirium Psychomotor Subtypes in Individuals with Hip Fracture. J Am Geriatr Soc 2015; 65:970-976 
This writing business. Pencils and what-not. Over-rated if you ask me. Silly stuff.

Nothing in it.

- Eeyore

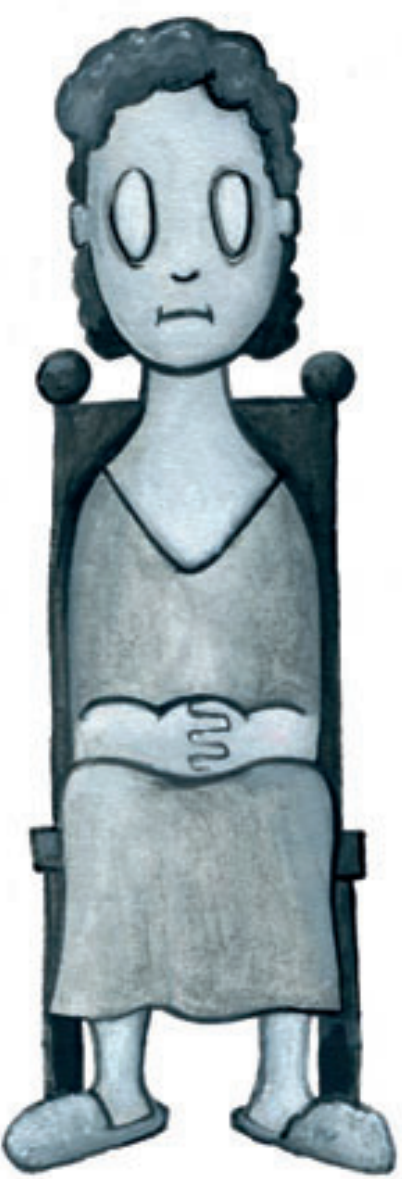




\section{Addenda}

Summary Samenvatting Valorisation Acknowledgements / dankwoord Publications \& curriculum vitae 

Summary 
This dissertation provides insight into the daily practice concerning the recognition and management of delirium in older (aged 65 years and over) hospitalised patients. Delirium occurs in between 29 and 64\% of older hospitalised patients and can have severe negative consequences, such as institutionalisation or death. Understanding how delirium is being recognised and managed in daily practice is essential, as well as assessing the effectiveness of different interventions (such as an educational intervention or a medication review), as this knowledge can help us improve the care for these patients. First, we performed a systematic literature review on all the available delirium detection instruments (chapter 2). Second, we evaluated how often delirium was diagnosed and how the different types of delirium were managed in the hospital (chapter 3). Third, we looked into the effectiveness of two different interventions (chapters 4 and 5), and lastly, we assessed the available evidence for current pharmacological interventions (chapter 6).

Chapter one introduces this thesis and the topic of delirium. In it, the symptoms, risk factors, aetiology, management and prevention of delirium in older hospitalised patients are explained. Moreover, the aims and outline of this dissertation are introduced.

In chapter two, a systematic literature review is performed to find and evaluate the different instruments available for the detection of delirium in older hospitalised patients. More than 40 instruments are identified, of which only a few have been validated in several different studies. The results of this review indicate that the best diagnostic instrument is the Confusion Assessment Method (CAM), but the Nursing Delirium Screening Scale (NuDESC) is better and more feasible for everyday screening used by nurses. The Delirium Observation Screening score (DOS), which is used in most hospitals in the Netherlands, is also good and feasible for everyday use by nurses.

A retrospective cohort study (chapter three), is performed to see how often delirium among older hospitalised patients was diagnosed, and how this was managed in daily practice. We found that delirium was diagnosed for $5.1 \%$ of the older patients. About three quarters of the delirious patients had a hyperactive delirium, while one quarter was diagnosed with hypoactive delirium. Delirious patients, irrespective of the type of delirium, were mainly managed pharmacologically, using antipsychotic medication. Previous research had found that delirium prevalence among older patients is between 29 and 64\%, of which the majority is of the hypoactive subtype. Moreover, according to national and international guidelines, the first step in delirium management should always be non-pharmacological.

To assess the effects of a medication review, another retrospective cohort study is performed in chapter four. Patients who had been delirious during their hospital stay and had received a medication review (performed by a geriatrician and a medical pharmacist), were compared to patients who had been delirious during 
their hospital stay a year previous, but had not received a medication review (this did not exist yet). The results show that patients who had received a medication review and were taking between zero and six medications prior to the medication review, had significantly shorter delirious episodes than comparable patients who had not received a medication review. Patients in the medication review group taking between seven and eleven medications also had shorter delirious episodes compared to patients who had not had a medication review, but this difference failed to reach statistical significance.

In chapter five we assess the effects of an educational intervention aimed at the nursing staff. The educational intervention focussed on the recognition and management of delirium in older hospitalised patients. First, the nursing staff of two different hospital wards were asked to fill out two questionnaires about delirium. Based on the answers to these questions we identified knowledge gaps and we then designed an educational programme based on these gaps in knowledge. The educational programme consists of an interactive one-hour lecture, and posters and flyers containing the gist of the lecture were placed on the wards. The main outcome measures which were used to assess the effectiveness of the educational intervention are: 1 . the percentage of patients who were screened at least once using the DOS; 2 . the number of patients for whom the DOS screening was carried out correctly (i.e. three times a day for at least three consecutive days); and 3. the percentage of patients for whom a geriatric consultation was requested. We found that after the educational intervention, the percentage of patients screened with the DOS increased significantly. Moreover, the number of patients who were screened at least three times a day for at least three consecutive days also increased. There was also an increase in the percentage of patients who received a geriatric consultation, though this increase failed to reach statistical significance.

Chapter six is a state-of-the-art article on the effectiveness of using antipsychotic medication for the management of hypoactive delirium in older hospitalised patients. Based on five high-quality reviews and three recent additional independent articles (which had not been included in the reviews due to recency), we assess the evidence for the use of antipsychotic medication for managing hypoactive delirium. We assess whether antipsychotic medication could have a positive effect on duration and severity of the hypoactive delirium and the experienced distress of delirious patients, on the length of hospital stay, and we looked at possible negative side-effects of antipsychotic medication. The results show that there are very few high-quality studies on the effectiveness of antipsychotics for delirium in general, and that there are no studies which focussed on antipsychotics for hypoactive delirium specifically. Moreover, none of the studies show a clear positive effect of antipsychotic medication on any of the above mentioned outcomes for delirium. Based on these results, we conclude that it is not advised to give antipsychotic medication to patients who have a hypoactive delirium and do not show any signs of psychomotor agitation. 
The main findings and conclusions of this dissertation are reflected upon in chapter seven. First, the diagnosis, subtypes and reporting of delirium are discussed, followed by guideline adherence and delirium management in daily hospital practice. Methodological considerations, including the study designs, study populations and the generalisability of the findings are discussed. Last, implications of these results for future research and for practice are discussed. 


Samenvatting 
Dit proefschrift verschaft inzichten in de dagelijkse praktijk van de herkenning en behandeling van delier bij oudere ziekenhuispatiënten (65 jaar en ouder). Delier komt voor bij 29 tot 64\% van de oudere ziekenhuispatiënten en kan ernstige negatieve gevolgen hebben, zoals opname in een zorginstelling of overlijden. Het is essentieel dat we begrijpen hoe delier in de dagelijkse praktijk herkend en behandeld wordt en wat de effectiviteit is van bepaalde interventies. Dit is belangrijk omdat we deze kennis kunnen gebruiken voor het verbeteren van de zorg omtrent delier bij oudere ziekenhuispatiënten. Als eerste hebben we een systematische literatuurstudie uitgevoerd naar alle beschikbare instrumenten voor de diagnostiek van delier (hoofdstuk 2). Als tweede hebben we onderzocht hoe vaak delier herkend wordt en hoe dit behandeld wordt in het ziekenhuis (hoofdstuk 3). Als derde hebben we de effectiviteit van twee verschillende interventies in de dagelijkse praktijk geëvalueerd (hoofdstukken 4 en 5). Tenslotte hebben we het bewijs voor de medicamenteuze behandeling van een stil delier beoordeeld (hoofdstuk 6).

Hoofdstuk één leidt het proefschrift in en geeft meer informatie over delier. In deze inleiding worden de symptomen, risico's, etiologie, behandeling en preventie van delier bij oudere ziekenhuispatiënten besproken. Tevens worden de doelen van dit proefschrift besproken en wordt er een overzicht van het proefschrift gegeven.

In hoofdstuk twee is een systematische literatuurstudie uitgevoerd naar de verschillende instrumenten die bestaan voor het herkennen van een delier bij oudere ziekenhuispatiënten. Er zijn meer dan 40 instrumenten gevonden, waarvan maar een handvol is gevalideerd in twee of meer studies. De resultaten van deze literatuurstudie wijzen erop dat de Confusion Assessment Method (CAM) het beste instrument is voor het vaststellen van een delier, maar de Nursing Delirium Screening Scale (NuDESC) is bruikbaarder voor de dagelijkse praktijk en kan beter door de verpleging gebruikt worden. In de meeste ziekenhuizen in Nederland wordt de Delirium Observatie Screening score (DOS) gebruikt: deze werd ook als goed en bruikbaar beoordeeld voor gebruik in de dagelijkse praktijk.

Hoofdstuk drie beschrijft een retrospectieve cohortstudie, waarin onderzocht wordt hoe vaak delier herkend wordt bij oudere ziekenhuispatiënten en hoe het delier vervolgens behandeld wordt. Wij vonden dat $5.1 \%$ van de oudere ziekenhuispatiënten gediagnosticeerd werd met een delier. Ongeveer drie kwart hiervan betrof patiënten met een geagiteerd delier en een kwart betrof patiënten met een stil delier. Delirante patiënten werden voornamelijk behandeld met antipsychotica, ongeacht het type delier. Eerder onderzoek heeft aangetoond dat delier voorkomt bij ongeveer 29-64\% van de oudere ziekenhuispatiënten, waarvan het merendeel een stil delier betreft. Bovendien hoort, volgens nationale en internationale richtlijnen, de eerste stap in het behandelen van een delier niet medicamenteus te zijn.

Een tweede retrospectieve cohortstudie, waarin onderzoek gedaan wordt naar de effectiviteit van een medicatierevisie, wordt beschreven in hoofdstuk vier. 
Patiënten die tijdens hun ziekenhuisopname delirant geworden waren en een medicatierevisie hadden gekregen, werden vergeleken met patiënten die een jaar eerder opgenomen waren en eveneens een delier ontwikkeld hadden tijdens hun ziekenhuisopname, maar die geen medicatierevisie ontvangen hadden omdat dit toen nog niet bestond. Uit de resultaten blijkt dat patiënten die tussen de nul en zes medicijnen slikken en een medicatiereview ontvangen hadden, significant korter delirant waren dan vergelijkbare patiënten die geen medicatierevisie ontvangen hadden. Patiënten die tussen zeven en elf medicijnen slikten en een medicatiereview ontvangen hadden waren ook wat korter delirant dan vergelijkbare patiënten die geen medicatiereview ontvangen hadden, maar dit verschil was niet statistisch significant.

In hoofdstuk vijf onderzoeken we de effectiviteit van een scholing voor verpleegkundigen over de herkenning en behandeling van delier bij oudere ziekenhuispatiënten. In de eerste stap werden verpleegkundigen van twee verschillende afdelingen gevraagd om twee vragenlijsten in te vullen: één over hun kennis van delier en één over de last die de zij ervaren bij het zorgen voor een delirante patiënt. Gebaseerd op de antwoorden op deze vragenlijsten is gekeken op welke gebieden de kennis van de verpleegkundigen onvoldoende was. De scholingsinterventie bestaat uit een één uur durende interactieve presentatie over delier en het plaatsen van posters en flyers met informatie over delier op de afdelingen. De uitkomsten waarmee de effectiviteit van de interventie gemeten werd zijn: 1. het percentage patiënten dat met de DOS gescreend werden op delier; 2. het aantal patiënten bij wie de DOS correct was afgenomen (d.w.z. drie keer per dag voor tenminste drie aaneensluitende dagen); en 3 . het percentage patiënten voor wie een geriatrisch consult werd aangevraagd. Wij hebben gevonden dat er na de interventie significant meer patiënten gescreend werden met de DOS. Bovendien werd bij meer patiënten de DOS correct uitgevoerd na de interventie dan voor de interventie. Tevens werd er voor een groter percentage patiënten een geriatrisch consult aangevraagd, hoewel deze toename niet statistisch significant was.

Hoofdstuk zes is een stand-van-zaken artikel waarin een overzicht gegeven wordt van alle literatuur aangaande de effectiviteit van het behandelen van een stil delier met antipsychotica bij oudere ziekenhuispatiënten. De resultaten zijn gebaseerd op vijf grote toonaangevende internationale literatuurstudies en drie recente individuele studies. Wij hebben gekeken of antipsychotica effectief zijn in het verkorten van de duur en ernst van een stil delier en het door de patiënt ervaren lijden als gevolg van het stille delier. Verder hebben we gekeken naar de effecten van antipsychotica op de duur van de ziekenhuisopname van de patiënt en naar de mogelijke bijwerkingen van antipsychotica. De resultaten laten zien dat er erg weinig studies van goede kwaliteit zijn uitgevoerd naar de effectiviteit van antipsychotica bij delier in het algemeen, en dat er geen studies bestaan, goede of slechte, uitgevoerd bij specifiek patiënten met een stil delier. Bovendien is in geen van de studies een sterk positief effect van antipsychotica gevonden op de bovengenoemde uitkomsten voor patiënten met een (stil) delier. Gebaseerd op 
deze resultaten concluderen wij dat het niet aan te raden is om antipsychotica te geven aan patiënten met een stil delier, bij wie geen sprake is van agitatie of psychomotore onrust.

In hoofdstuk zeven bespreken we de voornaamste uitkomsten van dit proefschrift en wordt er gereflecteerd op de uitkomsten en conclusies. Als eerste worden de diagnostiek, subtypes en behandeling van delier bij oudere ziekenhuispatiënten besproken, gevolgd door een bespreking van het opvolgen van de richtlijnen en de behandeling van delier in de dagelijkse praktijk in een ziekenhuis. Vervolgens worden de methodologische overwegingen besproken, het ontwerp van de verschillende studies, de onderzoekspopulaties en de generaliseerbaarheid van de resultaten. Als laatste worden de implicaties van de resultaten van dit proefschrift voor de praktijk en toekomstig onderzoek besproken. 




\section{Valorisation}


A valorisation is "the process of creating value from knowledge, by making knowledge suitable and/or available for social (and/or economic) use and by making knowledge suitable for translation into competitive products, services, processes and new commercial activities" (Promotiereglement Maastricht University, 2016). Basically, this means that the "knowledge" obtained in this dissertation should be made available and understandable for others to put it to (good) use, either to make the world a better place or to make money. The university has provided a series of topics to guide us through this valorisation addendum.

\section{Societal and/or economic relevance}

Results from my research have shown that delirium recognition and guideline adherence for the management of delirium among older hospitalised patients is poor. This poor recognition and management leads to potentially increased percentage of people who develop a delirium, and to longer or more severe delirious episodes. Results from my dissertation have also shown that some interventions that are already incorporated in daily practice can reduce the duration of delirious episodes and increase in the use of screening for delirium in older patients. These results are both of societal and economic relevance: the adverse outcomes associated with delirium can be very severe, and the health care costs associated with delirium are high.

With regards to the societal effects: older patients suffering a delirium during hospitalisation run a $62 \%$ increased risk of mortality within twelve months after the hospital admission compared to patients who did not develop a delirium. ${ }^{26}$ Other adverse outcomes include prolonged hospital stay, slower functional recovery, cognitive decline, institutionalisation after hospital discharge, greater chance of hospital readmissions, recurring delirium, and onset of dementia or deterioration of pre-existent dementia. ${ }^{27-30}$ Patients also suffer from emotional or psychological distress after a delirious episode. ${ }^{31}$ After recovering from a delirium, patients have been found to suffer from symptoms of depression, anxiety, and fear, ${ }^{32,} 33$ and delirium may be a risk factor for the development of post-traumatic stress disorder (PTSD). ${ }^{34}$ Taking into account that between 29 and $64 \%$ of older hospitalised patients develop a delirium, the results of this dissertation could potentially (positively) affect a lot of patients: shorter delirious episodes are beneficial for swifter functional and cognitive recovery. This dissertation also shows that delirium is severely under-recognised and under-reported. This knowledge is important because it shows there is a problem regarding recognition and adequate reporting of delirium, which may spark a discussion on the need to improve this. Early recognition of delirium may help in providing timely and adequate management, and reporting of delirium will help substantially in identifying patients with an increased risk of delirium during future hospital admissions, prompting the use of interventions to prevent delirium. 
With regard to the economic effects: delirium places a serious financial burden on the health care system. Longer and more hospital admissions, long-term care institutionalisation, dementia; these are all adverse outcomes with high financial consequences. It has been calculated that delirium among older hospitalised patients costs more than US $\$ 164$ billion a year in the USA, which is comparable to the healthcare costs for falls or diabetes mellitus. ${ }^{35} \mathrm{~A}$ German study team calculated what a patient with a hyperactive delirium actually costs the hospital excluding the costs for re-admissions or institutionalisation. ${ }^{36}$ They found that patients with a hyperactive delirium costs on average $€ 1200$,- more than patients with no delirium, based on extra time spent by physicians and nurses, longer hospital stay, and additional medication costs. Thus, the medication review as described in this dissertation, which has found to shorten the delirious episodes for certain groups of patients, could potentially decrease health care costs for these patients. Moreover, the educational intervention described in this dissertation, lead to an increase in the frequency of screening for delirium, which could potentially identify more patients at risk, prompting preventive measures and thus preventing delirium.

\section{Target groups}

The results of this dissertation are relevant to various groups of people outside the research community, mainly health care professionals, health care insurers, educators and policy makers, though they may also be important for older patients and their family members.

The results regarding the recognition and reporting of delirium, as well as the management of delirium using both non-pharmacological and pharmacological interventions, such as a medication review or the use of antipsychotic medication is important for health care professionals (i.e. nursing staff and physicians). These results show that the guidelines for detecting, reporting and managing a delirium are often not adhered. The guidelines are based on scientific research and expert opinions, and were made to improve the care for patients who have a delirium or run an increased risk of developing one. Adhering to the guidelines may help to prevent delirium, or, as is the case for the medication review, shorten the duration of a delirium in certain patient populations. Fewer and shorter delirious episodes decrease workload and stress for nursing staff and could potentially decrease the hospital stay for some patients, making place for new patients who need medical care. This could in turn lead to a decrease in health care costs, which make the results of this dissertation interesting for health care insurers.

Delirium has been made and indicator of hospital quality (kwaliteitsindicator ziekenhuiszorg) by the Dutch health care inspectorate (Inspectie GezondheidsZorg, IGZ). As such, the results regarding recognition and guideline adherence are of interest to policy makers and hospital boards, as decreasing delirium and increasing guideline adherence can increase the quality of care as assessed by the 
IGZ. This could lead to a higher ranking in the national ranking of hospitals in the Netherlands, and increase overall quality of care for older patients. This research is also of interest to policy makers, because the results show that just having the guidelines in place and facilitation delirium recognition by implementing the use of the Delirium Observation Screening scale (DOS) is not enough to warrant good delirium care. Policy makers could use these results to increase the focus of delirium, for example by making delirium a mandatory part of the list of possible side effects to be discussed with all older patients undergoing elective surgery.

\section{Products, services, processes, activities or commercial activities}

All my research was bundled into this dissertation, which is freely available upon request and includes, among others, an educational intervention, which can be used to educate health care professionals on how to recognise and manage delirium in a hospital setting. Moreover, this dissertation shows that simply providing delirium education for the nursing staff is not enough to create the needed change in clinical practice to improve delirium care: this should go hand-in-hand with education for the medial staff, an increased focus on delirium throughout the hospital, a mandatory thorough medication review for all older (at-risk) people admitted to hospital, and preferably providing good information to patients and family members (especially before elective surgery) on what a delirium is, how it can be prevented, and what the consequences are. Thus, a factsheet for non-medically trained people (laymen), was developed, which can be used to educate patients and family members, thus increase the focus of delirium throughout the hospital.

Furthermore, the knowledge created by the research presented in this dissertation has been disseminated in different ways over the past few years. Scientific articles have been published, which are available open-source on-line; the results were presented to fellow researchers and to health care professionals during national and international conferences. Knowledge was also disseminated through the newsletters of the Living Lab in Ageing \& Long Term Care (Academische Werkplaats Ouderenzorg Zuid-Limburg) and the Academic Collaborative Centre for Sustainable Care (Academische Werkplaats Duurzame Zorg). The latter also has a website with information regarding the research presented in this dissertation (https://www.duurzamezorgmaastricht.nl). Moreover, health care professionals (nurse practitioners, geriatricians, clinical pharmacologists) and policy makers within the hospital were asked to co-author or participate in several studies, thus incorporating them in the process of improving knowledge and guideline adherence for delirium recognition and management.

\section{Innovation}

The research in this dissertation focussed on the daily practice in a hospital setting, and so no innovative products, services, processes activities or commercial 
activities were created. This dissertation did, however, create an eye-opener for health-care professionals and policy makers, as the results made clear that just having guidelines does not mean that these guidelines are automatically adhered to. This could lead to policy makers having to find new and innovative ways to bring the guidelines concerning delirium to the attention of health care professionals: for example by making it mandatory to mention delirium as a possible (probable?) complication of hospital admission. This could increase awareness and sense of urgency among physicians. Moreover, by providing the previously mentioned factsheet and actively involving the patient and family members or carers in the process of delirium prevention and management, delirium recognition and care (thus guideline adherence) can be improved.

\section{Planning \& realisation}

With regards to the implementation of the interventions described in chapters four and five (the medication review and the educational intervention), these specific interventions were fairly location-specific, as they were designed to be implemented in the MUMC+ with the resources available at the MUMC+. However, a medication review does not need to be exactly as described in chapter four of this dissertation: it can easily be designed to fit in with the available resources of any hospital, both academic and non-academic, as and all hospitals employ specialists in internal medicine, geriatricians and/or a clinical pharmacist to carry out these medication reviews. The same can be said for the educational intervention described in chapter five of this dissertation: this was designed to fit in with the needs and possibilities of the nursing staff of two specific wards in the MUMC+. However, the intervention was designed in such a way that it could be tailored for the specific wards, and could also be tailored for different hospitals.

The results of this dissertation are most valuable for hospital policy makers. They should use the knowledge to improve delirium care in their hospitals, for example through delirium education for the health care professionals, implementing medication reviews for all older patients admitted to hospital, and by providing the necessary means to (pro-actively) educate patients and family members about delirium, before delirium happens. Placing flyers on a ward is not enough: these are often not given to the people who need them (or given only after a patient developed a delirium), and are often not replenished once the flyers are gone.

In conclusion: delirium is serious, common, costly and often overlooked or mismanaged. Pro-active education (or at least timely information) should be presented to patients and family. Health care professionals should be educated about delirium, its recognition, prevention and management. This will be beneficial for patients, family members, health care professionals, health care insurers and the financial state of our health care system in general. 



\section{Acknowledgements \\ Dankwoord}


$\mathrm{Na}$ vier jaar is het eindelijk zo ver: ik mag het meest gelezen hoofdstuk van dit proefschrift schrijven! Dit boekje was er nooit gekomen zonder de hulp van veel anderen. Sommige mensen hebben vooral met praktische zaken geholpen en andere mensen door me geestelijk of lichamelijk gezond te houden.

Allereerst wil ik mijn begeleidingsteam bedanken. Sandra, Ruud en Frans, dankzij jullie heb ik met veel plezier aan dit proefschrift gewerkt en wist ik altijd, na een nieuwe tegenslag, terug in het zadel te klimmen om vervolgens (wéér) een nieuw onderzoeksvoorstel te bedenken. Jullie stonden altijd met de neuzen dezelfde kant op en ik heb echt veel geluk gehad met jullie als team. Sandra, jij had altijd tijd voor een snelle vraag of een (langere) babbel, zowel over mijn onderzoek als over vakanties, huizen en honden. Jij was er vanaf het begin van overtuigd dat ik alles op tijd af zou krijgen en gelukkig heb je gelijk gekregen! Ik sta versteld van wat jij allemaal doet op een dag en vraag mij af waar je de tijd vandaan haalt. Nu ben je net hoogleraar geworden, gefeliciteerd! Ruud, jij stimuleerde mij altijd om zelf na te denken in plaats van direct antwoord te geven op mijn vragen. Tijdens onze overleggen was er altijd ruimte voor persoonlijke vragen en verhalen en je had altijd tips voor vakantiebestemmingen omdat je werkelijk overal al bent geweest! Daarbij verbaasde het mij elke keer weer wanneer je binnen een paar uur een volledig document had nagekeken; jouw oog voor zowel de grote lijn als voor detail zijn onevenaarbaar. Frans, jij was inhoudelijk de drijvende kracht achter het team, en ik heb tijdens onze discussies veel geleerd over delier, met name in het latere stadium van mijn promotietraject. Je wist altijd de juiste vragen te stellen om te zorgen dat ik weer nét iets dieper ging nadenken. Wubbo, jij was geen officieel lid van mijn team, maar dankzij jou kon ik toch snel aan de slag in het ziekenhuis.

Graag wil ik de leden van de beoordelingscommissie, prof. Richard Koopmans, prof. Bianca Buurman, dr. Hans Fiolet, prof. Roos van der Mast en prof. Jos Schols hartelijk danken voor het lezen en beoordelen van dit proefschrift.

Frans Manders en Sjef Bremen, jullie hebben mij altijd geholpen om (snel) aan de juiste data te komen. Jullie weten het misschien niet, maar zonder jullie had dit proefschrift er heel anders uitgezien en had ik veel meer stress gehad.

Noor, Jannic, Evelien, Liesbeth en de overige deelnemers aan de wekelijkse "grote visite ouderengeneeskunde", dankzij jullie is mijn (praktische) kennis over delier bij ouderen, maar ook mijn kennis over de ouderengeneeskunde in het algemeen, enorm gegroeid. Jullie doen super goed werk en zijn echt een top team! Ron, bedankt voor de gezelligheid tijdens conferenties in San Francisco en Orlando, alle discussies over onderzoek doen, delier, ouderengeneeskunde en (zelf gebrouwen) bier.

Irma en Maartje, mijn lieve, toffe, stoere 0.098 roomies! Bij ons op de kamer werden er drie dingen altijd vol overgave gedaan: werken, kletsen en koffiedrinken. Ik denk liever niet aan hoeveel liter koffie wij met zijn drieën in de afgelopen vier jaar gedronken hebben, lekker warm tegen de verwarming aan in de winter, of 's zomers buiten in het zonnetje. Jullie waren altijd bereid te luisteren wanneer ik weer eens tekeer ging over referenten of mijn frustraties met de METC en het 
CTCM, maar jullie hielpen ook met praktische zaken zoals problemen met word of Engelse vertalingen en zinnen die niet liepen. Daarbij heb ik veel van jullie geleerd over voetbal, bloemencorso en gemene Russen die met waterbekertjes gooien. Ik hoop dat we het drinken van wijntjes en speciaalbiertjes blijven doen nadat we allemaal weg zijn hier.

Ik wil ook de rest van de HSR collega's bedanken voor alle leuke uitjes, lunches, wandelingen, congressen en soepmomenten. De "dames van het secretariaat", Suus, Brigitte, Janet en Joanna: de drijvende kracht achter onze vakgroep! Dank voor al jullie hulp met mijn duizend-en-één vragen de afgelopen jaren.

I also want to thank all the $\mathrm{PhD}$ academy people, especially (but not exclusively) the Improv group and those involved in the play (coincidentally called "confusions"). You are all mad and amazing! There is so much enthusiasm, happiness, craziness and mad ideas in this group. Thanks Sanne, Dorijn, Nola, Mehrdad, Martijn, Jessie, Adam, Elisa, Nienke, Sanne R, Fred, Frederik, Frederik, JoAnne, Paola, Mahdi, Norbert, Zubin, Mare, Anna, Bas, Tobi, Geraldine, Mueid, Masha, Juan, Dixon, Madalin, Heather, Gregor and soooo many more. Keep up the good vibes!

Jelena, jij had altijd een positieve invloed op mijn humeur en werklust, maar een negatieve invloed op mijn lever. Dank je voor al die maandagavonden vol rode wijn en jazz, voor alle lunches met witte wijn en vooral voor al je goede advies en je luisterend oor. $\mathrm{Nu}$ ben je je nieuwe leven in Utrecht begonnen, en wordt je hier in Maastricht wel gemist! Hvala.

Martijn, ik kan mij geen betere huisgenoot wensen dan jij! Je weet me altijd te verbazen met alles wat je doet en de energie en toewijding die je overal in stopt (salsa, klimmen, breien, klussen aan je auto, kasten bouwen, lekker koken, slechte films en nog slechtere muziek (afgewisseld met verbazend goede muziek)). Bovendien sta je altijd voor iedereen klaar en ben je nooit te beroerd om iemand te helpen. We zullen je missen als je ooit besluit te verhuizen.

The ladies 8+: Annemarie, Ester, Catharina, Simone, Seda, Sophie, Lisa, Fei Yian, Aline, Cecile, Thera, Fanny, Coco en Marie, onder de leiding van de enige echte Coach Titus: dankzij jullie bleef ik niet alleen mentaal, maar ook fysiek gezond! Dank voor alle gezellige woensdagavonden op de ergo, zaterdagochtenden, wedstrijden, etentjes en trainingsweekenden. Good busy girls! Roeien is één van de beste keuzes die ik de afgelopen vier jaar gemaakt heb.

Rosalinde, jij had altijd een luisterend oor wanneer ik frustraties moest spuien en weet me ook altijd weer aan het lachen te krijgen! Matt, samen met Rosa weten jullie als geen ander de nerd in mij wakker te maken en elke keer wat groter te laten groeien. Ook wist jij, Matt, als geen ander mijn ideeën te vertalen naar de prachtige illustratie die nu te vinden is op de kaft van mijn proefschrift! Ik hoop dat er nog heel veel wandel- en gameweekenden zullen volgen.

Caroline en Anouk, mijn oudste vriendinnen! jullie staan altijd voor mij klaar wanneer dat nodig is en samen hebben we nogal wat meegemaakt. Car, ik ben blij dat jij mijn paranimf wil zijn en Anouk, jij de "hoffotograaf". Zonder jullie waren de 
afgelopen 13 jaar stuk minder leuk geweest, en ik hoop dat de komende 13 net zo leuk worden! :)

Marguerite en Maarten: dankzij jullie heb ik de rust en ruimte (en thee en wijn) gehad om de kerstvakantie door te kunnen werken zodat ik dit proefschrift op tijd af kon krijgen. Dank voor jullie gastvrijheid en de warmte waarmee jullie mij vanaf de eerste dag omarmd hebben.

Mijn lieve, gezellige en drukke familie: mam, pap, Philip, Annemarie (en Ruud, Liselotte en Carlijn) en Marjolein. Pap en mam, jullie wil ik bedanken voor alle vrijheid en steun die jullie ons altijd gegeven hebben in het maken van onze eigen keuzes: jullie steunen ons door dik en dun en steken nooit onder stoelen of banken hoe trots jullie op ons zijn. Af en toe kreeg ik via de post ineens een uitgeknipt artikel over delier dat in de krant of een tijdschrift stond of een tip voor een uitzending op TV. Jullie zeggen altijd wel: "min keer min maakt plus", maar plus keer plus maakt ook plus! Philip, Annemarie en Marjolein, ik vind het mooi dat we, ondanks dat we zo verschillend zijn, zo hecht zijn en altijd voor elkaar klaarstaan. Ik vond het ook altijd fijn wanneer jullie mij een hart onder de riem staken met oppeppende woorden en goede advies! Uitspraken zoals "ik snap niet dat iemand er vrijwillig voor kiest om vier jaar lang een scriptie te schrijven" en "ik val bij de titel van je artikelen meestal al in slaap" gaven mij de moed om er weer tegen aan te gaan ;) (maar jullie lazen wel altijd mijn stukken wanneer ik er om vroeg!). Annemarie, ik hoop dat Liselotte en Carlijn dit boekje ooit openslaan en denken: "ik wil ook vier jaar lang scriptie schrijven"!

Marjolein, jou wil ik apart nog even bedanken dat je mijn "independent physician" was. Dankzij jou weet ik dat excenteratio totalis geen Harry Potterspreuk is en dat salbutamol ipatroprium valt in de categorie "long". Over het gebruik van haloperidol bij delirante patiënten zijn we het niet altijd eens, maar ik heb ook wel makkelijk praten van achter mijn computer.

Roald, wie had ooit gedacht dat de METC tijdens mijn promotietraject nog ergens goed voor zou zijn. Jij hebt me de afgelopen drie jaar ontzettend gesteund en ik heb heel veel aan je gehad. Jij was er altijd voor me: zowel emotioneel als inhoudelijk, door me te steunen, stoom af te laten blazen, de juiste vragen te stellen en mee te denken over onderzoeksmogelijkheden en -vragen. Je drang naar vakanties, avontuur en reizen is er één die ik deel, en ik hoop dat we nog heel veel gaan beleven samen! Ik weet dat je niet zo van clichés houdt, maar ik doe het hier lekker toch (het is tenslotte mijn boek!): jij bent mijn rots in de branding en ik houd van jou! 




\section{Publications}

$\&$ curriculum vitae 


\section{Publications}

E.L. van Velthuijsen, S.M.G. Zwakhalen, G.I.J.M. Kempen, F.R.J. Verhey (2018). Behandeling van een stil delier: wel of geen antipsychotica? (accepted for publication in the Nederlands Tijdschrift voor Geneeskunde).

E.L. van Velthuijsen, S.M.G. Zwakhalen, R.M.J. Warnier, T. Ambergen, W.J. Mulder, F.R.J. Verhey, G.I.J.M. Kempen (2018). Can education improve clinical practice concerning delirium in older hospitalised patients? Results of a pre-test posttest study on an educational intervention for nursing staff. BMC Medical Education, 18(1):59.

E.L. van Velthuijsen, S.M.G. Zwakhalen, E. Pijpers, L.I. van de Ven, T. Ambergen, W.J.M. Mulder, F.R.J. Verhey, G.I.J.M. Kempen (2018). Effects of a medication review on delirium in older hospitalised patients: a comparative retrospective cohort study. Drugs \& Aging, 35(2):153-161.

E.L. van Velthuijsen, S.M.G. Zwakhalen, W.J.M. Mulder, F.R.J. Verhey, G.I.J.M. Kempen (2017). Detection and management of hyperactive and hypoactive delirium in older patients during hospitalization: a retrospective cohort study evaluating daily practice. International Journal of Geriatric Psychiatry, doi: 10.1002/gps.4690, e-pub ahead of print.

E.L. van Velthuijsen, S.M.G. Zwakhalen, R.M. Warnier, W.J.M. Mulder, F.R.J. Verhey, G.I.J.M. Kempen (2016). Psychometric properties and feasibility of instruments for the detection of delirium in older hospitalized patients: a systematic review. International Journal of Geriatric Psychiatry, 31(9), 974-989.

R.M. Warnier, E. van Rossum, E.L. van Velthuijsen, W.J. Mulder, J.M. Schols, G.I.J.M. Kempen (2016) Validity, reliability and feasibility of tools to identify frail older patients in inpatient hospital care: a systematic review. Journal of Nutrition and Healthy Aging, 20(2), 218-230. 


\section{About the author}

Eveline Louise van Velthuijsen was born on February 15th 1987 in Breda, the Netherlands. She moved to Spain at the age of eight, where she completed her primary school and the first year of high school. At the age of thirteen she moved back to the Netherlands, where she completed her high school education at Laar \& Berg in Laren (NH) in 2005 and then studied the Bachelor Psychology at Utrecht University and at the Universidad Autónoma de Madrid, in Madrid, Spain. During her Bachelor, Eveline was a volunteer for the "buddy project" where she mentored a person with schizophrenia. After her Bachelor degree, she had a gap year during which she worked at a psychiatric institution (Altrecht) and travelled through Europe. Upon her return, Eveline continued to study the Master Clinical Neuropsychology at Utrecht University. In the years following her graduation, Eveline worked as a research assistant for different projects at Yulius centre for mental health care in Rotterdam and later at the psychiatry department of the University Medical Centre Utrecht (UMCU) on the BIG-genetics project; about people with bipolar (manic-depressive) disorder. In 2014 she started her PhD trajectory at Maastricht University under the supervision of Prof. dr. Sandra Zwakhalen, Prof. dr. Gertrudis (Ruud) Kempen and Prof. dr. Frans Verhey, which she completed within four years. This PhD project focussed on the recognition and management of delirium in older hospitalised patients and resulted in this dissertation. Eveline is currently employed as an old-age psychologist in a nursing home. 